Check for updates

Cite this: Phys. Chem. Chem. Phys., 2020, 22, 19401

Received 20th May 2020, Accepted 7th August 2020 DOI: $10.1039 / \mathrm{d} 0 \mathrm{cp} 02741 \mathrm{c}$ rsc.li/pccp

\section{Advances and challenges for experiment and theory for multi-electron multi-proton transfer at electrified solid-liquid interfaces}

\author{
Ken Sakaushi, (D) *a Tomoaki Kumeda, (D) ${ }^{a}$ Sharon Hammes-Schiffer, (D) $*^{b}$ \\ Marko M. Melander (D) *c and Osamu Sugino (D)*d
}

\begin{abstract}
Multi-electron, multi-proton transfer is important in a wide spectrum of processes spanning biological, chemical and physical systems. These reactions have attracted significant interest due to both fundamental curiosity and potential applications in energy technology. In this Perspective Review, we shed light on modern aspects of electrode processes in the 21st century, in particular on the recent advances and challenges in multistep electron/proton transfers at solid-liquid interfaces. Ongoing developments of analytical techniques and operando spectrometry at electrode/electrolyte interfaces and reliable computational approaches to simulate complicated interfacial electrochemical reactions enable us to obtain microscopic insights about these complex processes, such as the role of quantum effects in electrochemical reactions. Our motivation in this Perspective Review is to provide a comprehensive survey and discussion of state-of-the-art developments in experiments, materials, and theories for modern electrode process science, as well as to present an outlook for the future directions in this field.
\end{abstract}

\footnotetext{
${ }^{a}$ Center for Green Research on Energy and Environmental Materials, National Institute for Materials Science, Namiki 1-1, Tsukuba, Ibaraki 305-0044, Japan. E-mail: sakaushi.ken@nims.go.jp

${ }^{b}$ Department of Chemistry, Yale University, 225 Prospect Street, New Haven, CT 06520-8107, USA. E-mail: sharon.hammes-schiffer@yale.edu

${ }^{c}$ Nanoscience Center, Department of Chemistry, University of Jyväskylä, P.O. Box 35 (YN), FI-40014, Jyväskylä, Finland. E-mail: marko.m.melander@jyu.fi

${ }^{d}$ The Institute of Solid State Physics, the University of Tokyo, Kashiwanoha 5-1-5, Kashiwa, Chiba 277-8581, Japan. E-mail: sugino@issp.u-tokyo.ac.jp
}

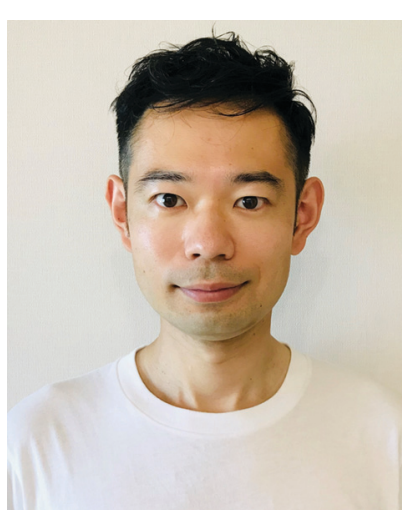

Ken Sakaushi
Ken Sakaushi is a Senior Researcher at the National Institute for Materials Science (NIMS), Tsukuba, Japan. He studied physics (BSc, 2008) and chemistry (MSc, 2010) at Keio University, and he completed his PhD at IFW Dresden/TU Dresden in 2013 with a DAAD fellowship. Then, he joined Max Planck Institute of Colloids and Interfaces on a fellowship from Max Planck Society. In 2015, Ken joined NIMS as a Tenured Member. His research focuses on various fundamental aspects of electrode process. Besides academic activities, he loves playing piano, reading books, and playing football. Ken has been recognized by awards such as the ISE Travel Award for Young Electrochemists, PCCP Prize, and PCCP Emerging Investigator. Further information: https://sites.google.com/site/sakaushiken/.

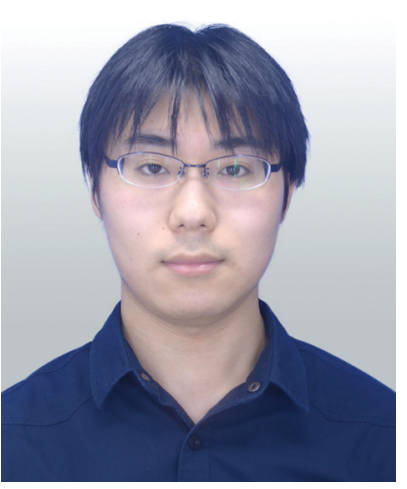

Tomoaki Kumeda
Tomoaki Kumeda received his PhD from Chiba University, Japan, in 2020 under the supervision of Associate Professor Masashi Nakamura. He was a recipient of a JSPS Research Fellowship for Young Scientists from 2018 to 2020. He is currently a postdoctoral researcher at National Institute for Materials Science, Japan. His research interests focus on microscopic structure of electrode/electrolyte interfaces and mechanism of electrocatalytic reactions on single crystal surfaces. 


\section{Scope of this perspective review}

Electrode processes based on multi-electron, multi-proton transfer at solid-liquid interfaces are attracting widespread interest ranging from fundamental scientific issues to engineering aimed at establishing next-generation energy storage/ conversion devices. In this Perspective Review, the cutting-edge advances and challenges of modern electrode process science are surveyed and discussed by experts in this field: Ken Sakaushi (Sections 1-3), Tomoaki Kumeda (Section 4: operando IR/Raman surface-electrochemical spectrometry), Sharon Hammes-Schiffer

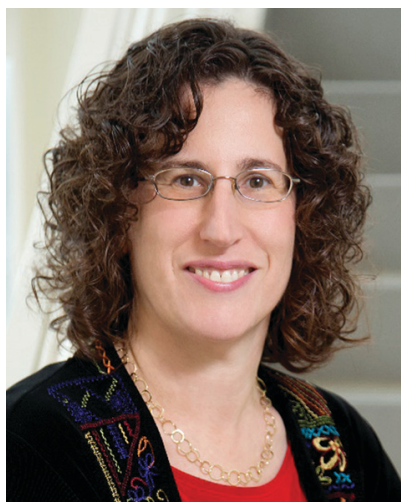

Sharon Hammes-Schiffer

Sharon Hammes-Schiffer received her $B A$ in Chemistry from Princeton University in 1988 and her PhD in Chemistry from Stanford University in 1993, followed by two years at AT\&T Bell Laboratories. She was the Clare Boothe Luce Assistant Professor at the University of Notre Dame from 1995-2000 and then became the Eberly Professor of Biotechnology at The Pennsylvania State University until 2012, when she became the Swanlund Professor of Chemistry at the University of Illinois Urbana-Champaign. Since 2018, she has been the John Gamble Kirkwood Professor of Chemistry at Yale University. Her research centres on the investigation of charge transfer reactions, protoncoupled electron transfer, nonadiabatic dynamics, and quantum mechanical effects in chemical, biological, and interfacial processes. Her work encompasses the development of analytical theories and computational methods, as well as applications to experimentally relevant systems.

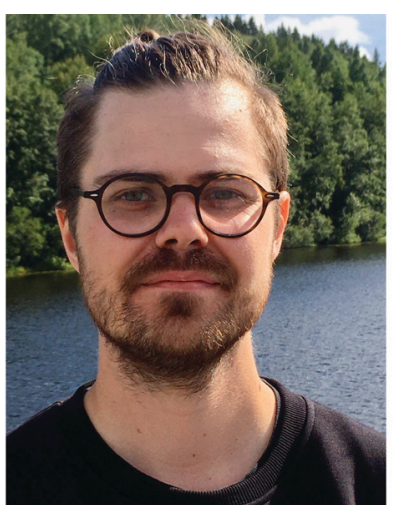

Marko M. Melander
Marko M. Melander is an Academy of Finland Postdoctoral researcher at the University of Jyväskylä, Finland. Before obtaining his current position in 2017, he obtained a PhD in physical and computational chemistry from Aalto University in 2015 after which he joined the Atomic Scale Modelling and Materials group at DTU. His research focuses on development and application of theory and computational methods to study (proton-coupled) electron transfer in electrochemical systems, heterogeneous catalysis of complex systems, and multiscale modelling.

(Section 5: heterogeneous electrochemical proton-coupled electron transfer theory), Marko M. Melander (Section 6: grand canonical ensemble for electrochemical thermodynamics and reaction rates), and Osamu Sugino (Section 7: quantum manybody theory for understanding electrode processes).

The scope of this Perspective Review focuses on the modern forms of electrode process science, including a wide range of different aspects, i.e. experimental approach, electrode material design, modeling, and first-principles calculations. We begin the discussions with the dawn of modern electrochemistry and then move to experimental approaches including kinetic analysis, material design and operando electrochemical spectrometry. After these experimental topics, we move to theoretical topics covering the recent advancements and applications of proton-coupled electron transfer theory at electrode/electrolyte interfaces, the grand canonical ensemble approach to simulate electrochemical reaction rates, and quantum many-body theories aimed toward understanding microscopic mechanisms of electrochemical reactions.

The aims in this Perspective Review are the following: (1) circulating modern knowledge of electrochemistry for a wide spectrum of scientists including graduate students, (2) summarizing the state-of-the-art developments in experimental and theoretical aspects of electrode process science, and (3) discussing current theoretical and computational approaches including classical, quantum mechanical, adiabatic, and nonadiabatic treatments in electrochemical kinetics.

\section{Prologue}

Nature acquired one of the most efficient energy conversion systems on the earth based on electron transfer (ET), proton transfer (PT), and proton-coupled electron transfer (PCET) (Fig. 1).

As energy conversion in biological systems keeps on attracting interest since the finding of the proton transfer mechanism in the cytochrome system, ${ }^{1,2}$ it is well-known that this efficient

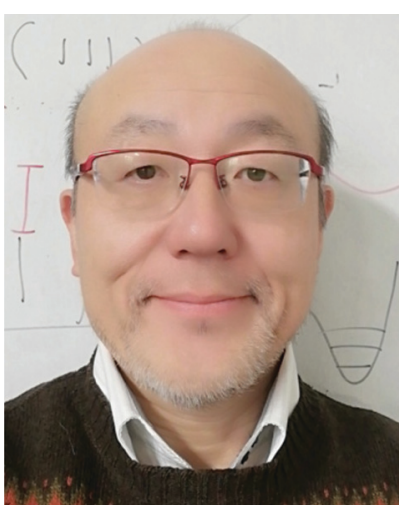

Osamu Sugino
Osamu Sugino was born in Fukushima Prefecture, Japan in 1961. He obtained his BSc (1984), $M E$ (1986) and PhD (1989) degrees from the University of Tokyo. He was an employee (1989-2002) at NEC corporation, an associate professor (2002-2017) at the University of Tokyo. Since 2017, he has been a professor at the University of Tokyo. He has worked on the theory of condensed matter physics, in particular, the firstprinciples calculation of nonadiabatic dynamics and electrode dynamics. 


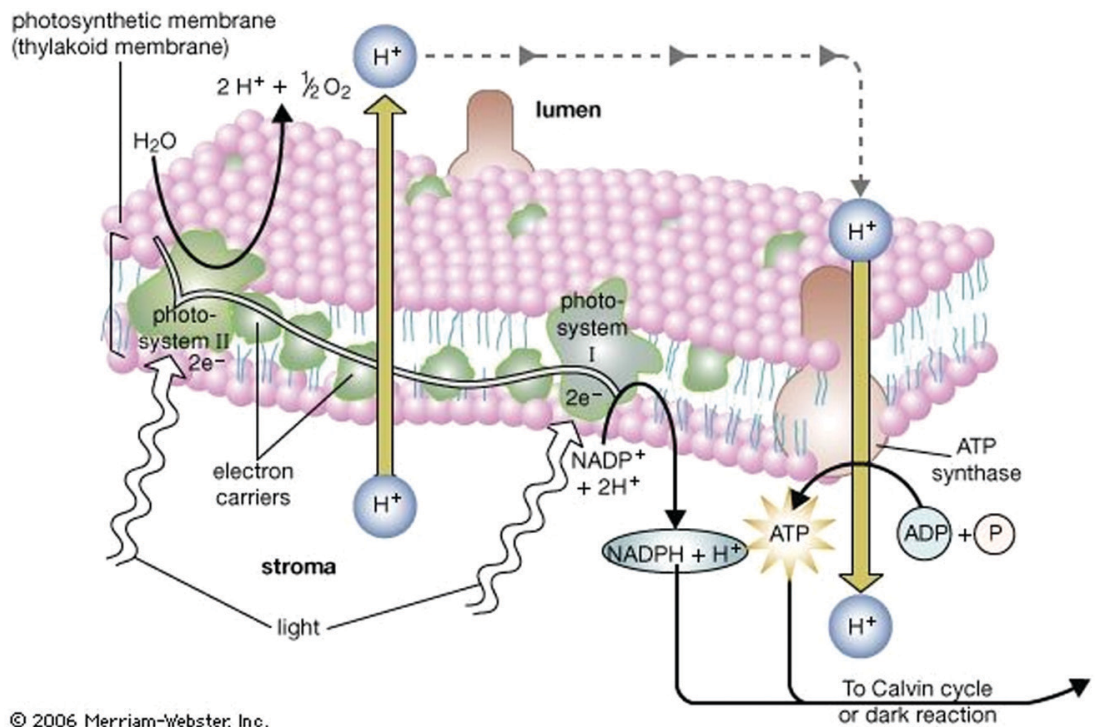

Fig. 1 The light reaction of photosynthesis as highly efficient proton-based energy conversion system. Proton transfer is a key of this system. Photosystem II obtains replacement electrons from water molecules, resulting in their splitting into proton $\left(\mathrm{H}^{+}\right)$and oxygen atoms. The oxygen atoms combine to form molecular oxygen $\left(\mathrm{O}_{2}\right)$, which is released into the atmosphere. $\mathrm{H}^{+}$is pumped into the lumen by electron acceptor molecules. The flow of $\mathrm{H}^{+}$back across the photosynthetic membrane provides the energy needed to drive the synthesis of the energy-rich molecule of adenosine triphosphate (ATP). High-energy electrons, which are released as photosystem I absorbs light energy, are used to drive the synthesis of nicotinamide adenine dinucleotide phosphate (NADPH). Photosystem I obtains replacement electrons from the electron transport chain. ATP provides the energy and NADPH provides the hydrogen atoms needed to drive the subsequent photosynthetic dark reaction, or Calvin cycle. The figure and caption are adopted from Encyclopædia Britannica ${ }^{\mathbb{R}}$ by courtesy of Encyclopædia Britannica, Inc., copyright 2006; used with permission: URL https://www.britannica.com/ plant/plant/Photosynthesis\#/media/1/463192/66097, Access Date 29th Dec. 2019. The original caption was modified by KS.

system is a relay of multi-electron/-proton transfer reactions (Fig. 2). ${ }^{3-5}$

Therefore, it is not surprising that there were a large number of researchers trying to understand the mechanisms of surface electrode processes involving biological synthesis of adenosine triphosphate (ATP) and adenosine diphosphate (ADP) in the early 1970s. ${ }^{6,7}$ As is obvious for ET, the modern view of PT can be described under the laws of quantum mechanics; there are active discussions on the connections between nuclear quantum effects (NQE) and microscopic
A Wieland's
dehydrogenases

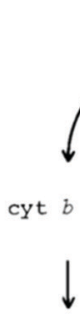

Keilin's cytochromes Warburg's
oxidase $\longrightarrow$
B

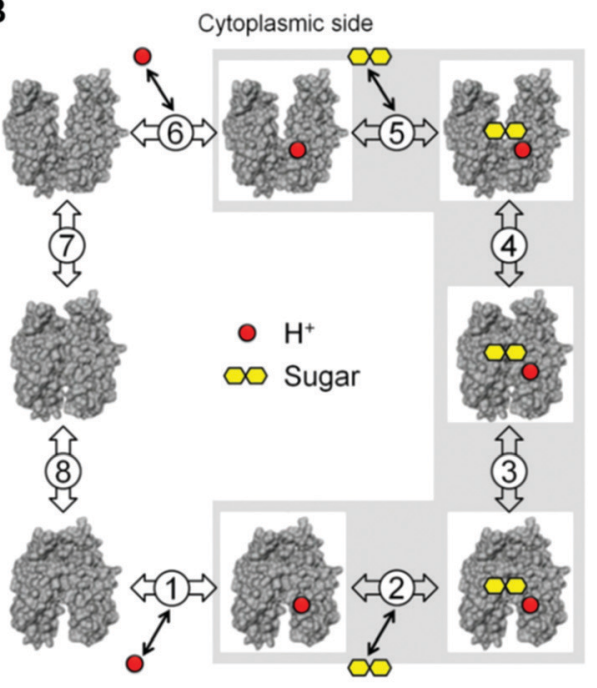

Periplasmic side

Fig. 2 Classical and modern models for proton-related multistep reactions in proteins. (A) David Keilin's chemically simple respiratory chain concept. The panel and the caption were adopted from the ref. 4, with the permission from the Nobel Foundation. Copyright: The Nobel Foundation (2020). (B) Kinetic scheme for galactoside/ $\mathrm{H}^{+}$symport, exchange, and counterflow in LacY. The panel was reprinted from the ref. 5. Copyright (2020) National Academy of Sciences of the United States of America. 

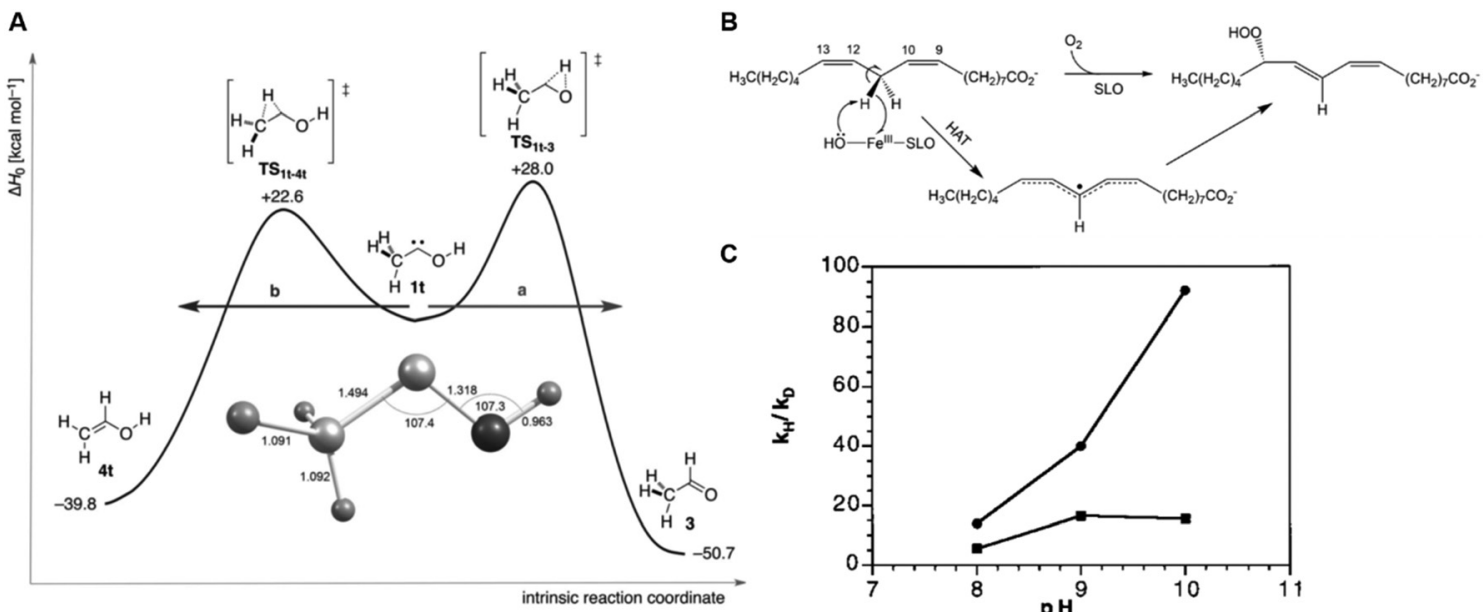

mechanisms in biological systems ${ }^{8-13}$ and organic chemistry (Fig. 3). ${ }^{14-16}$

Similar to these examples from chemistry and biology, electrochemists were eager to adopt the recently developed quantum theory, and there is a rich history on utilizing quantum mechanics in electrode processes. ${ }^{17-31}$ The marriage of electrochemistry and quantum mechanics in the early 20th century was a fascinating and beautiful event in science because this opened the door to the establishment of modern electrode process science, which is the advanced framework of classical electrochemistry developed by many researchers since the 18th century. ${ }^{32,33}$ The birth of modern electrode process science was triggered by R. W. Gurney at Cavendish Laboratory/Trinity Hall, University of Cambridge in 1931: quantum mechanics was applied to interpret a microscopic mechanism of an elementary act of the hydrogen evolution reaction. ${ }^{17}$ In this report, the proton discharge at an electrode surface was described by state-of-the-art physical concepts at that time, including the Franck-Condon principle and Fermi-Dirac statistics. This arose from the communications with Condon and Morse during Gurney's stay at Palmer Physical Laboratory, Princeton University. ${ }^{34,35}$ This illustrates that the modern ideas of electrode processes emerged from strong interactions between physics and chemistry; therefore, this subject has considerable physics character by nature even in its modern form of electro"chemistry".

After Gurney's work on applying quantum mechanics to an electrode process, a wide spectrum of quantum theories were proposed to describe microscopic electrode processes, such as bond-stretching (inner-sphere) model based theories, ${ }^{20,36-38}$ and solvent-reorganization (outer-sphere) model based theories. ${ }^{21-24,39-43}$ From this point, the application of quantum mechanics to modernize electrochemistry may have influenced the work of three Nobel Prize laureates, i.e. P. Mitchell, R. A. Marcus and J. B. Goodenough. ${ }^{4,44,45}$ Here we skip explaining the detailed history of these quantum electrochemical theories and their developments because one of the authors already summarized the aforementioned issues in recent reports. ${ }^{46,47}$ It is clear that we have plenty of basic theories to consider microscopic electrode processes; however, the development of quantum mechanical descriptions of electrode processes had become almost stagnant after the late 1970s. One of the main reasons was the extraordinarily high complexity of surface electrochemical processes at solid-liquid interfaces, requiring advanced simulation methods, theoretical concepts, and wellestablished surface-sensitive spectrometry to observe reaction dynamics.

Fortunately, with present technology, we have enough supercomputers and equipment to make progress, as already shown in heterogeneous surface science at solid/gas interfaces. ${ }^{48-52}$ In fact, several fascinating theoretical and computational approaches for electrode processes containing quantum effects in electron and proton transfer reactions were proposed since the late 1990s. ${ }^{53-66}$ Therefore, we believe that the time for further advancement of quantum electrode process science, a scientific topic having a long tradition, has come. It is our hope that we can show the importance, challenges, and excitement in this field to the readers in order to encourage further developments of this highly interdisciplinary topic in the 21st century. Studying and understanding how quantum mechanical effects such as nuclear and electron tunneling and non-adiabaticity control electrochemical properties will advance electrode process science and hopefully lead to materials with increased control of selectivity and activity.

\section{Experimental observations of quantum effects in electrode processes}

\subsection{Introduction to the observation of quantum effects in multi-electron/-proton transfer electrode processes}

As the lightest chemically relevant particles, electrons display quantum effects and ET is always a quantum process (Fig. 4). ${ }^{67}$ 

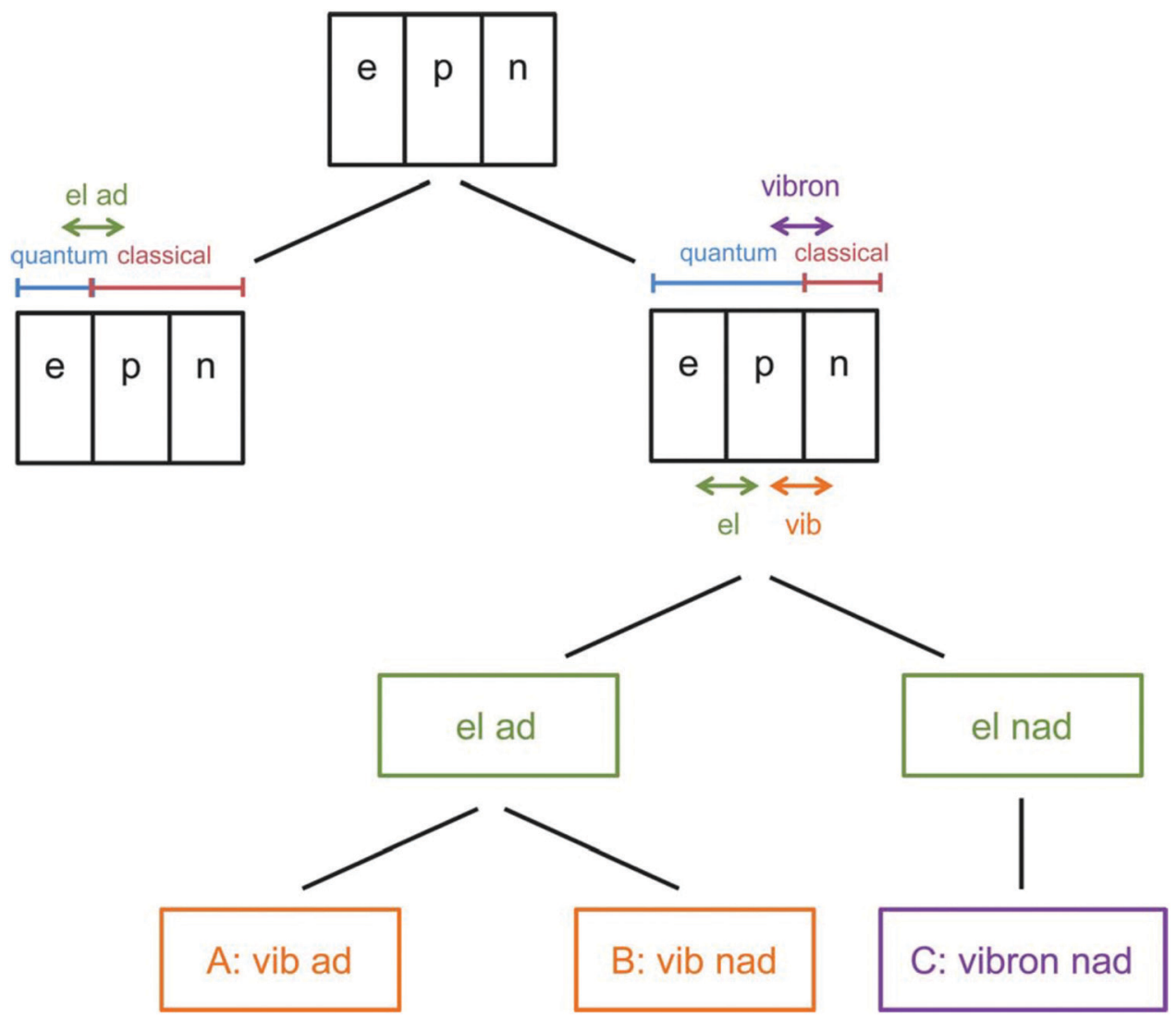

Fig. 4 Schematic picture of the classification scheme for proton transfer and general PCET reactions. The abbreviation "el ad" denotes electronically adiabatic, where the electrons respond instantaneously to the motions of all nuclei. This left branch is associated with standard electronic structure calculations. The right branch depicts the situation in which the electrons and transferring proton are treated quantum mechanically, and all other nuclei are treated classically. The abbreviations are defined as follows: "el" denotes electronically, "vib" denotes vibrationally, "vibron" denotes vibronically, "ad" denotes adiabatic, and "nad" denotes nonadiabatic. This branch is subdivided into "el ad" and "el nad", referring to the degree of electron-proton nonadiabaticity, and the "el ad" case is subdivided further into "vib ad" and "vib nad." The figure and caption were adopted with permission from the ref. 67. Copyright (2012) Royal Society of Chemistry.

Similarly, protons should also be treated as quantum mechanical particles, and PT and concerted proton-electron transfer (CPET) are the key processes for understanding quantum effects in electrode processes (see the state-of-the-art theory on PCET in Sections 5-7). The details on CPET can be found in the previous report. ${ }^{68}$ If a PT for the slowest step in a multistep reaction displays quantum effects such as tunneling or zero-point energy effects, this reaction can be regarded as a quantum-dominated process. Therefore, quantum effects in PT and CPET are an important focus of investigations in quantum electrode processes.

Although the first experimental suggestion of quantum mechanical effects in PT/CPET in an electrode process was reported by Bawn and Ogden in $1934,{ }^{18}$ and many leading electrochemists, such as Conway, ${ }^{69-76}$ tried to confirm this phenomenon, the experimental observation of quantum electrode processes was hampered by several issues:

(1) there was no general approach to determine the ratedetermining step (RDS) of multistep electrochemical reactions until the establishment of the Parsons' relation. ${ }^{77}$

(2) analytical equations to diagnose quantum processes such as the electrochemical kinetic isotope effect (EC-KIE) were less developed.
(3) there was no easily-accessible and reliable technology to obtain ultrapure grade heavy reagents, especially water, until quite recently. $^{78-80}$

Especially, the issue (3) was quite important because ultrapure heavy water is indispensable to observe a reliable EC-KIE, which is often taken as an indicator for NQEs. For these reasons, observing the signs of quantum electrode processes was enabled only quite recently. ${ }^{46,47,81}$

To investigate NQEs in electrode processes, first, we should identify the RDS in the corresponding multi-electron multiproton transfer reaction. The first step is to classify a possible RDS as one of the following three cases: ET, PT, or CPET (Fig. 5). ${ }^{82-85}$ Practically, the classification can be possible by applying the combination of the Parsons' relation to an experimentally obtained the transfer coefficient $(\alpha)$ and the EC-KIE analysis. In other words, the Parson's relation is able to check whether ET is involved in a RDS, and the EC-KIE analysis is able to indicate whether PT is involved in this RDS or not.

We can confirm which reaction step is a possible RDS by checking the Tafel slope: the relation between the Tafel slope and the RDS is well studied for steps in typical reactions, such as the hydrogen evolution reaction and oxygen reduction reaction. ${ }^{86,87}$ 


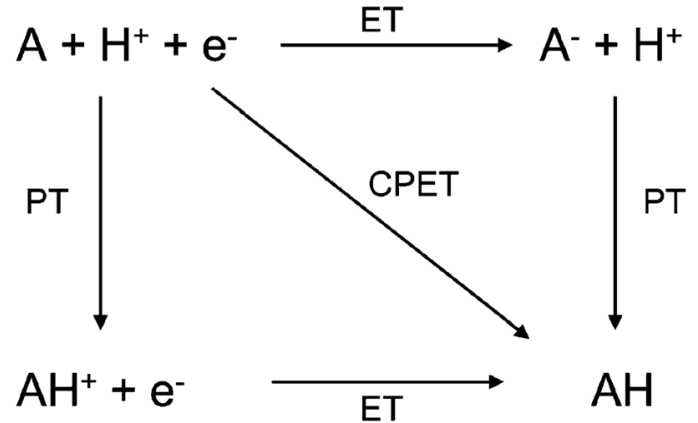

Fig. 5 Square scheme for proton-coupled electron transfer. ET = electron transfer, $\mathrm{PT}=$ proton transfer, $\mathrm{CPET}=$ concerted proton-electron transfer. The figure and caption were adopted with permission from the ref. 85. Copyright (2013) Royal Society of Chemistry.

The detailed method and equations to obtain the Tafel slope and $\alpha$ are summarized in the recent report. ${ }^{46}$ Furthermore, this author notes that there are issues to apply this method to several reactions so far, for instance the $\mathrm{CO}_{2}$ reduction reaction because of the difference between surface and bulk $\left[\mathrm{H}^{+}\right] \cdot{ }^{88,89}$ Once $\alpha$ is obtained, this can be substituted into the equations to calculate the EC-KIE $\left(K^{\mathrm{H} / \mathrm{D}}\right)$, which is based on the ratio of the reaction rate constant for hydrogen and deuterium. ${ }^{46}$ Typically, very large KIEs, $K^{\mathrm{H} / \mathrm{D}} \gg 1$ are used as indicators for NQEs. However, using the KIE as the sole indicator for discerning quantum effects is difficult as even non-adiabatic nuclear tunneling could result in KIEs close to unity if excited vibronic states play a significant role. ${ }^{90,91}$

$$
\begin{gathered}
K_{\mathrm{ORR}}^{\mathrm{H} / \mathrm{D}}(\eta)=\frac{C_{0, \mathrm{D}}}{C_{0, \mathrm{H}}} \frac{j_{\mathrm{k}, \mathrm{H}}(\eta)}{j_{\mathrm{k}, \mathrm{D}}(\eta)} \\
K_{\mathrm{ORR}}^{\mathrm{H} / \mathrm{D}}(\eta)=\frac{\left[\mathrm{D}^{+}\right] C_{0, \mathrm{D}}}{\left[\mathrm{H}^{+}\right] C_{0, \mathrm{H}, \mathrm{H}}(\eta)} \frac{j_{\mathrm{k}, \mathrm{D}}(\eta)}{K_{\mathrm{ORR}}^{\mathrm{H} / \mathrm{D}}(\eta)=\frac{\left[\mathrm{D}^{+}\right]}{\left[\mathrm{H}^{+}\right]} \frac{j_{\mathrm{k}, \mathrm{H}}(\eta)}{j_{\mathrm{k}, \mathrm{D}}(\eta)}}
\end{gathered}
$$

With these equations, more detailed discussions are accessible, for example, it was indicated that the size of electrocatalysts can affect to quantum electrode processes (Fig. 6). The detailed discussions of these equations are available in the previous reports. ${ }^{46,47,92}$ These equations are easy to handle and were successfully applied to analyze a wide spectrum of electrocatalysis, ${ }^{78,80}$ and also have been used to observe quantum electrode processes. ${ }^{46,47,81}$ On the other hand, the pictures of reaction mechanisms obtained by these equations are relatively ambiguous or too simplified. The equations including additional effects for ORR (eqn (1)-(3)) were provided in the recent report to demonstrate more details of the microscopic mechanism of electrode processes. ${ }^{92}$

\subsection{What do we understand about quantum electrode processes so far?}

Starting from the most important point, based on previous investigations, we can conclude that quantum effects, for instance zero point energy (ZPE) and tunneling, are key in electrode processes at solid-liquid interfaces. ${ }^{46,47,81,92}$ Therefore, quantum effects

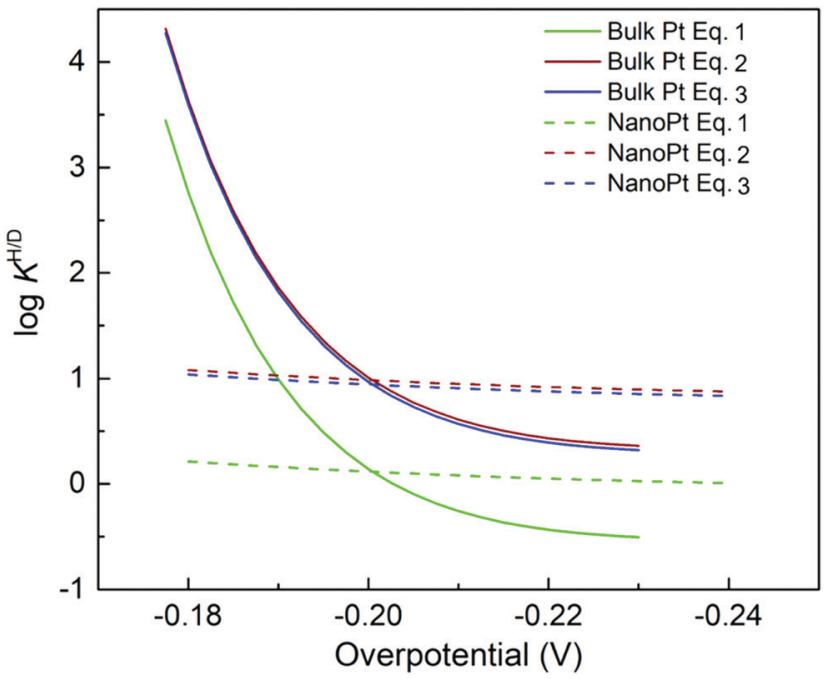

Fig. $6 \log K^{H / D}$-overpotential diagrams for ORR with different analytical equations. The solid and dotted lines respectively indicate bulk Pt and nano Pt electrodes. The different features in bulk Pt and nano Pt electrodes are obtained. This can suggest a size-dependent quantum-to-classical transition in electrode processes. The figure was adopted with permission from the ref. 92. Copyright (2020) Royal Society of Chemistry.

could be universally important for multi-electron multi-proton transfer electrode processes. For example, a strong indication of quantum proton tunneling was observed in a system composed of Pt electrode in an alkaline condition. In this system, a large EC-KIE was observed in a low overpotential region but vanished at high overpotentials. This was interpreted as a transition from a quantum proton tunneling mechanism to a classical over-the-barrier mechanism. ${ }^{81}$ This observation suggests a simple mechanistic picture, i.e. a high activation barrier allows tunneling to generate a current only at a lower overpotential condition, but this barrier height is lowered for higher overpotentials because of the BrønstedEvans-Polanyi (BEP) principle, and therefore a current via the classical TST path will be generated. However, this phenomenon can also be explained within a theoretical framework in which the proton is fully quantized for all overpotentials. ${ }^{93,94}$

Under the above simple picture, we can confirm the existence of a NQE in an electrode process. However, the important issue here is how to see a chemical or physical picture of a quantum electrode process that is solely dominated by quantum effects, especially "deep" tunneling (see Section 6 for details). As indicated by theoretical analyses (see Sections 5-7), ${ }^{81}$ the importance of quantum effects in electrode processes is valid, but confirming the microscopic mechanism and interpreting this phenomenon still presents huge challenges. Furthermore, previous reports showed the observation of a large difference in symmetry factors in $\mathrm{H}$ and $\mathrm{D}$ systems, ${ }^{46,95}$ which indicates a strong quantum effect; ${ }^{47}$ however, the reason for this observation still remains an open question. Several major challenges to understand the microscopic electrode mechanism are discussed in the next section.

\subsection{Key questions in experimental approach}

3.3.1. The activation model: Tafel or Marcus? Based on the traditional framework in physical electrochemistry, there are 
two main models to apply to electrode processes: the Tafel model predicts a linear $\log j_{\mathrm{k}}$ versus overpotential, and Marcuslike models predict quadratic $\log j_{\mathrm{k}}$ versus overpotential plots. Predictions of the Tafel model are consistent with the early bond-stretching model, while Marcus-like theories build on the reorganization of the environment facilitating ET/PT/CPET via tunneling. We note that Marcus-Hush-Chidsey (MHC) formalism, which incorporates the Fermi-Dirac distribution of electrons/holes into the classical Marcus theory, can show perfect agreements between its modified rate equation and the curved Tafel plot. ${ }^{96,97}$ However, this distinction is somewhat artificial, and a more general perspective is a combination of concepts from these two models. For instance, DFT-based approaches are mainly based on models resembling the bond-stretching model and often neglect the environmental reorganization but still can exhibit a quadratic dependence of the current on the electrode potential (Fig. 7). On the other hand, models utilizing the solvent reorganization concept can result in (nearly) linear $\log j_{\mathrm{k}}$-overpotential relations for large reorganization energies. Indeed, modern theoretical and computational approaches treat both the bond stretching (inner-sphere) and solvent reorganization (outer-sphere) in a consistent manner. Therefore, determining the activation mode or the "correct" theoretical framework from Tafel plots alone seems futile.

While the microscopic activation mechanism cannot be understood from Tafel plots, it is well-known that a wide spectrum of different electrocatalytic reactions show a change in Tafel slope associated with a change of potential (Fig. 8) ${ }^{78,81,98}$ This change in Tafel slope due to potential is suggested to be linked to a change of reaction mechanism, for instance, from the four-electron ORR mechanism to another mechanism (Fig. $8 \mathrm{C}$ and E). ${ }^{46,78,80,81}$ or potential-dependent changes in the surface coverages. ${ }^{99}$ To understand an activation mechanism and associated phenomena, we need further details from experimental and theoretical investigations at solid-liquid electrode processes at the molecular level (see Sections 5-7). Especially, the modern view of proton-coupled electron transfer theory (see Sections 5 and 6) can shed light on this fundamental yet unsolved question in electrochemistry. Moreover, a clear interpretation of activation mechanisms of electrode processes will help to discover electrocatalysts having better or comparable activity to Pt-based materials or to find a way to solve present issues in non-Pt electrocatalysts (see the "In silico electrocatalyst design" in Section 7). ${ }^{78,80,100-107}$

3.3.2. How to confirm further details on quantum electrode processes? There are several quantum electrode processes that are difficult to describe from a microscopic mechanistic perspective or to confirm experimentally. The first key issue is the anomalous $K^{\mathrm{H} / \mathrm{D}_{-}} \eta$ relation, which was observed for the HER on a $\mathrm{Au}$ electrode in alkaline conditions (the Volmer step as the RDS). This system showed a potential-dependent $K^{\mathrm{H} / \mathrm{D}}$, which increases with increasing driving force, i.e. overpotential. As a result, the proton transfer in this system has been suggested to transition from classical over-the-barrier TST behavior to quantum tunneling with an increase of driving force (Fig. 9). However, this type of potential-dependent kinetic isotope effect can also be described
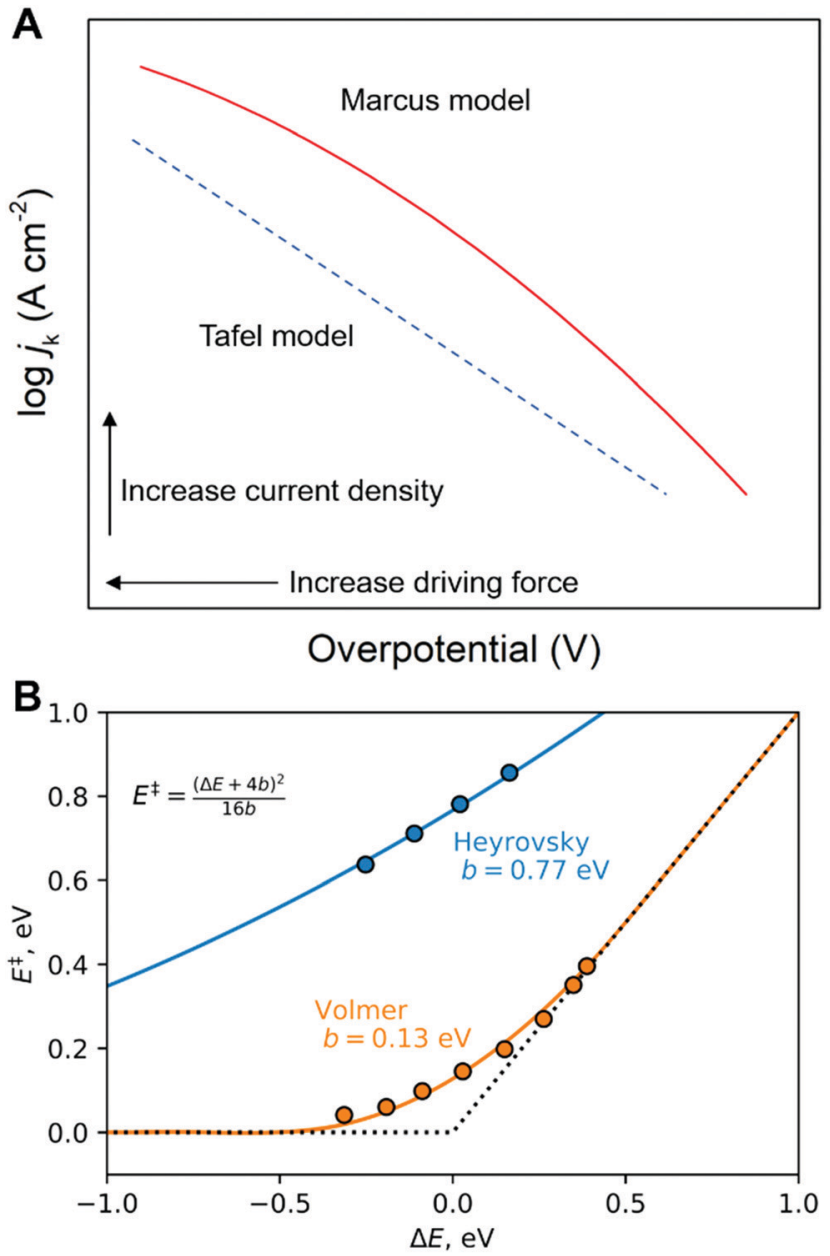

Fig. 7 Tafel and Marcus models in $\log j_{\mathrm{k}}$ vs. overpotential diagrams. (A) Simulated $\log j_{\mathrm{k}}-\eta$ diagrams. The dotted blue line is calculated by the Tafel model, i.e. $j_{\mathrm{k}}=j_{0} \exp (A \eta)$. The solid red line is calculated by the Marcus model, i.e. $j_{\mathrm{k}}=j_{0} \exp (B+C \eta)^{2}$. The $A, B$, and $C$ are arbitrary coefficients. (B) Example of Tafel-like and Marcus-like features obtained by a DFT calculation. Panel B was adopted with permission from the ref. 65. Copyright (2020) American Chemical Society.

with a vibronically nonadiabatic PCET theory in which the transferring hydrogen nucleus is quantized over the entire range of overpotentials (see Section 5). ${ }^{93}$ Moreover, a unified framework with a quantized proton can interpolate between adiabatic TST behavior and nonadiabatic behavior, ${ }^{94}$ and in some cases a single reaction can span both regimes for different distances from the electrode surface. In addition, an increase of EC-KIE along with an increase of driving force is unusual from the point of the BEP principle, which is another indication of NQEs. ${ }^{108}$ Although there are a few proposals to explain this feature, ${ }^{46,47}$ the microscopic mechanism is unclear and KIEs can result from several different properties of the system. ${ }^{109}$

The second key issue is that some theoretical suggestions are challenges to observe experimentally. Recently, a vibronically nonadiabatic process was suggested to underlie the Volmer step at a $\mathrm{Au}$ electrode in acidic conditions. ${ }^{94}$ The theoretical calculation of the $\eta-\log j_{\mathrm{k}}$ plot using the vibronically nonadiabatic PCET theory agreed well with the experimental observations, 

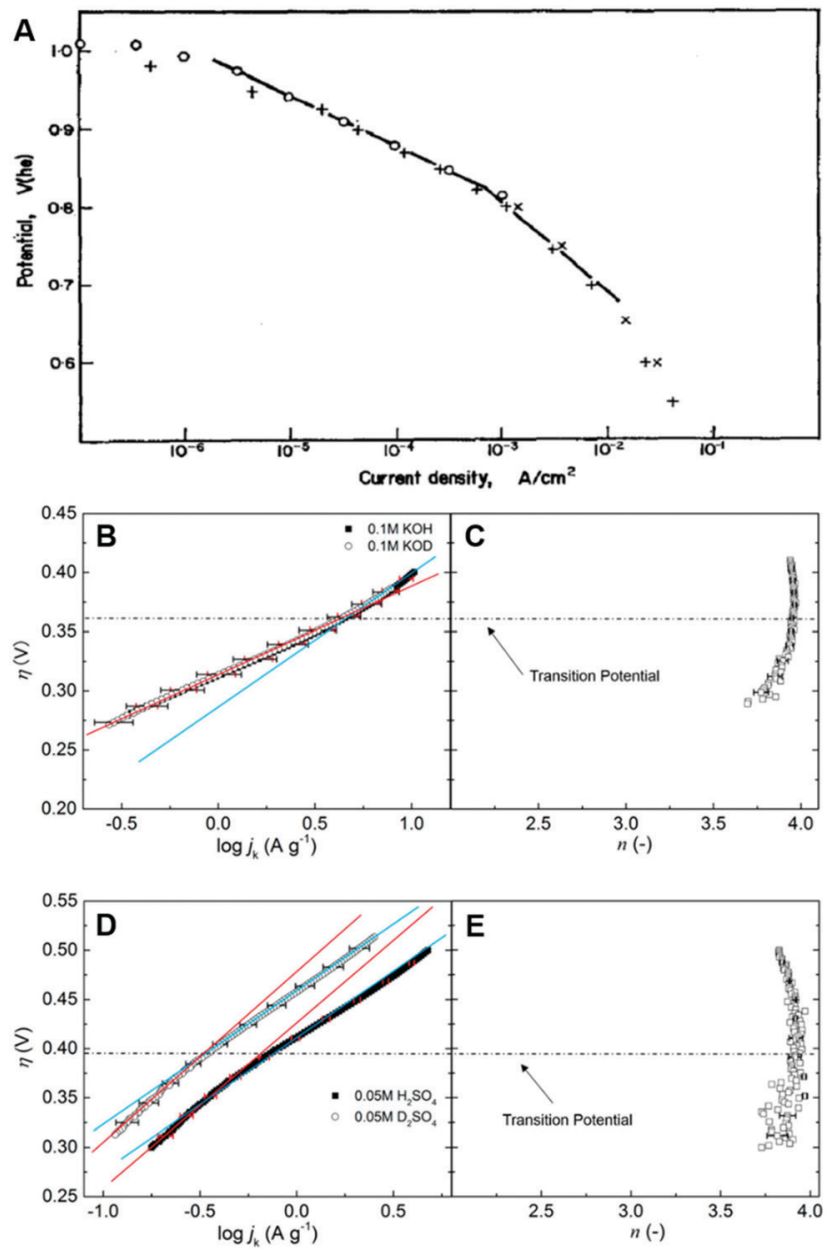

Fig. 8 Examples for Tafel slope change as function of potential. (A) ORR on Pt electrode in acidic conditions. (B) $\eta-\log j_{k}$ diagram for ORR on nonmetal carbon-based catalyst in ordinary and heavy water electrolyte: (C) electron transfer number ( $n$ ) according to (B) as a function of overpotential in alkaline electrolyte $\left(0.1 \mathrm{M} \mathrm{KOH}\right.$ in $\mathrm{H}_{2} \mathrm{O}$ and $0.1 \mathrm{M} \mathrm{KOD} \mathrm{in} \mathrm{H}_{2} \mathrm{O}$ ): (D) $\eta-\log j_{k}$ diagram for ORR on non-metal carbon-based catalyst in ordinary and heavy water based electrolyte; (E) $n$ according to $(D)$ as a function of $\eta$ in acidic electrolyte $\left(0.05 \mathrm{M} \mathrm{H}_{2} \mathrm{SO}_{4}\right.$ in $\mathrm{H}_{2} \mathrm{O}$ and $0.05 \mathrm{M} \mathrm{D}_{2} \mathrm{SO}_{4}$ in $\mathrm{D}_{2} \mathrm{O}$ ). The red and blue lines indicate fits to obtain the Tafel slope as the slope of these lines in the lower (below 0.35 and $0.4 \mathrm{~V}$ in alkaline and acidic solutions, respectively) and higher overpotential regions, respectively. The panel $A$ and the corresponding caption are reprinted from the ref. 98, Copyright (1970), with permission from Elsevier. The panels B-E and the corresponding captions are reprinted and modified from the ref. 78 , Copyright (2018), with permission from American Chemical Society.

and therefore this theoretical work suggested that a nonadiabatic process could have an important role for electrode processes. However, the experimental $\eta-\log j_{\mathrm{k}}$ plot for a Au electrode in acidic conditions shows a linear $\eta-\log j_{\mathrm{k}}$ feature with a potentialindependent $K^{\mathrm{H} / \mathrm{D}}$ of $c a$. 3, which traditionally has been interpreted to indicate a small contribution from nuclear quantum effects and an over-the-barrier mechanism (Fig. 10). This indicates that the EC-KIE alone cannot be used as a fully satisfactory proof of NQEs and vibronically nonadiabatic tunneling.

The third key issue is clarifying when the NQEs are important and when a classical description of electrode processes is sufficient.

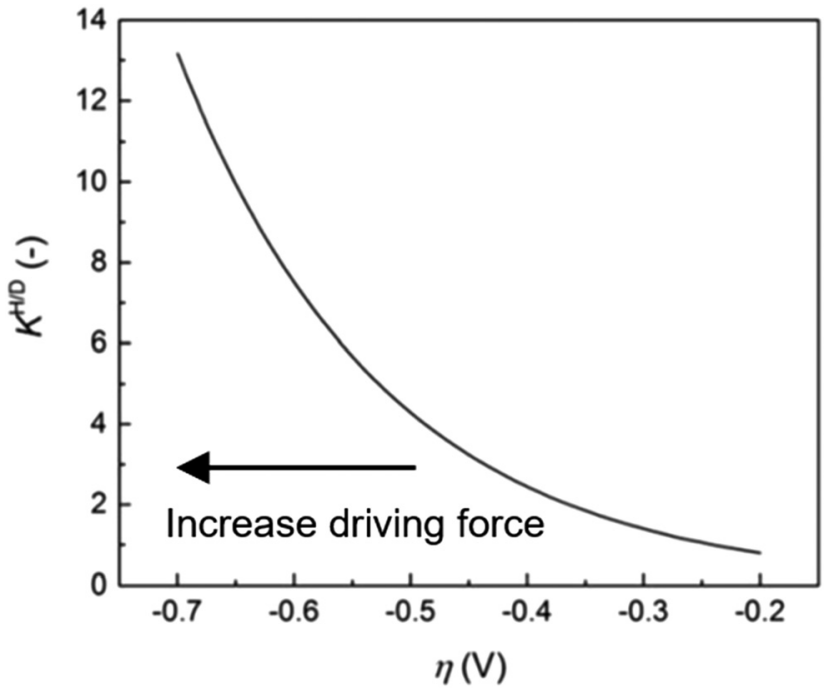

Fig. 9 Anomalous $K^{\mathrm{H} / \mathrm{D}}-\eta$ diagram. $K^{\mathrm{H} / \mathrm{D}}$ increases associated with an increase of driving force. The measurements were carried out for the HER on Au electrode in $0.1 \mathrm{M} \mathrm{KOH}$ in $\mathrm{H}_{2} \mathrm{O} / 0.1 \mathrm{M} \mathrm{KOD}$ in $\mathrm{D}_{2} \mathrm{O}$. The figure was reprinted from the ref. 46, Copyright (2020), with permission from the Royal Society of Chemistry.

The importance of quantum effects is most probably systemdependent and may change depending on $\mathrm{pH}$, solvent, anion additives in the electrolyte, etc. As shown in the following Sections, recent work on electrified surface systems successfully demonstrated various NQEs using computational and theoretical methods to investigate barrier structures, RDS, KIEs, and adsorption processes (see Sections 5-7 for the details). ${ }^{64,65,110}$ Therefore, these methods will play a significant role in solving the above issues in conjunction with experiments. EC-KIE 2experiments will likely offer much insight on mechanistic details and elementary steps of complex electrochemical reactions and provide a natural link between theory, computation, and experiments. If further advancement of computational approaches meets with operando electrochemical spectrometries (see Section 4) and state-of-the-art theories, for instance modern PCET theories (see Section 5), we will largely improve our fundamental knowledge of electrode processes.

Furthermore, recent advancements in materials science can also contribute to improving our understanding of electrode processes. For example, there are a variety of open discussions on porous electrodes. Recently, precisely prepared metallic porous electrodes were shown to be effective to study mechanisms of the $\mathrm{CO}_{2}$ reduction reaction (Fig. $11 \mathrm{~A}$ and B). ${ }^{111,112}$ The emerging material classes of metal-organic frameworks (MOFs) ${ }^{113-129}$ and covalent-organic frameworks (COFs) ${ }^{100,101,130-140}$ have also been shown to be powerful tools to investigate fundamental features of electrochemical reactions. For example, heterojunctions of twodimensional (2D) MOFs/COFs and metals are promising designer materials to tune the electrochemical properties of well-known electrode materials, such as Au or Pt, at solid-liquid interfaces. One of the two ideas here is based on the "classical" theories for electronic interactions at heterojunctions systems, such as the work of Bardeen, ${ }^{141}$ and of Cabrera and Mott, ${ }^{142}$ or Vol'kenshtein's theory for heterojunction catalysts, ${ }^{143}$ which has 


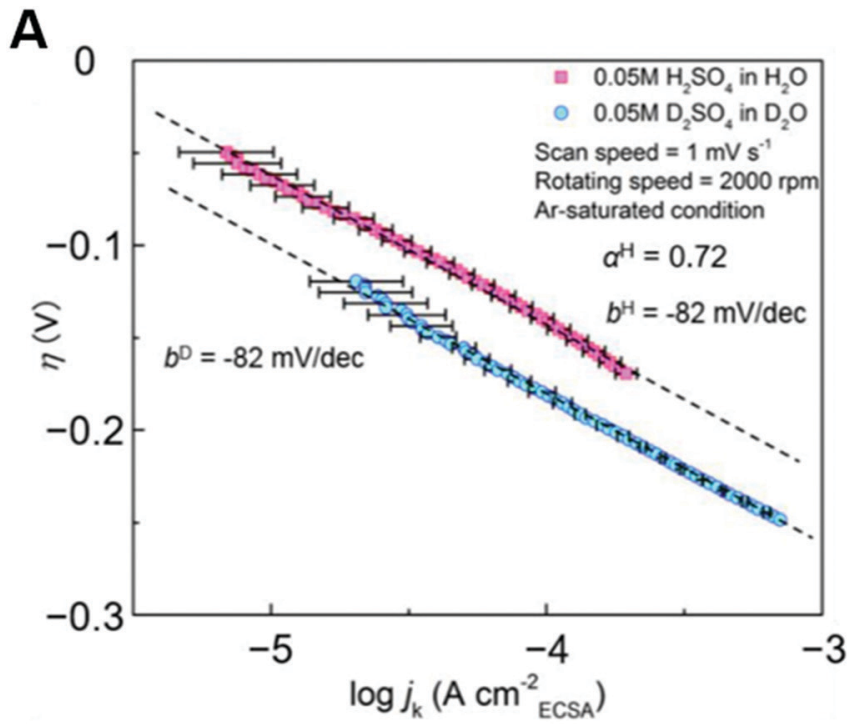

C

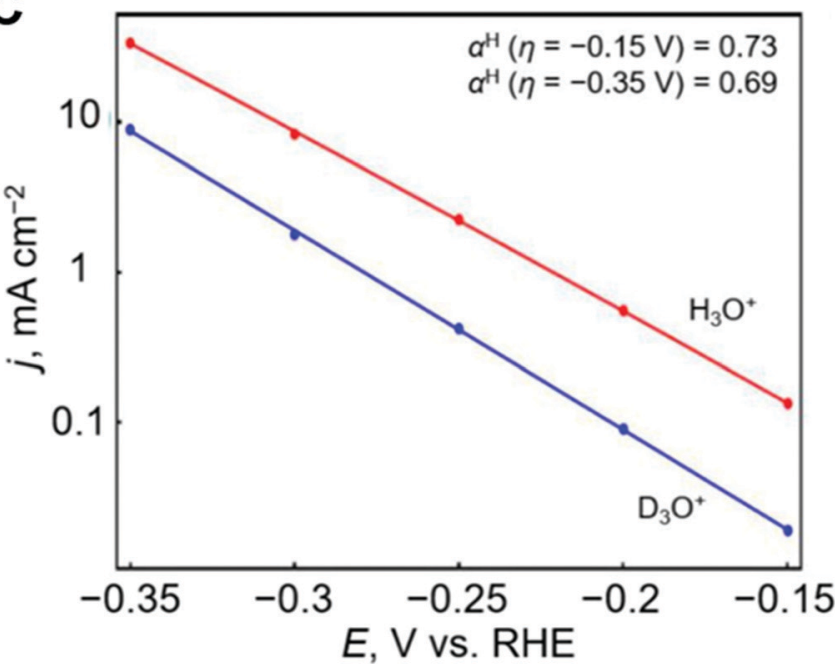

B<smiles>O=C1CCCCCC1</smiles>
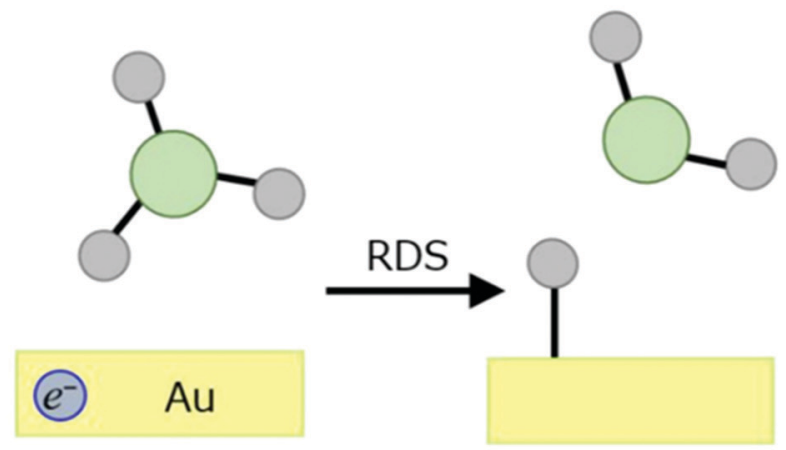

D

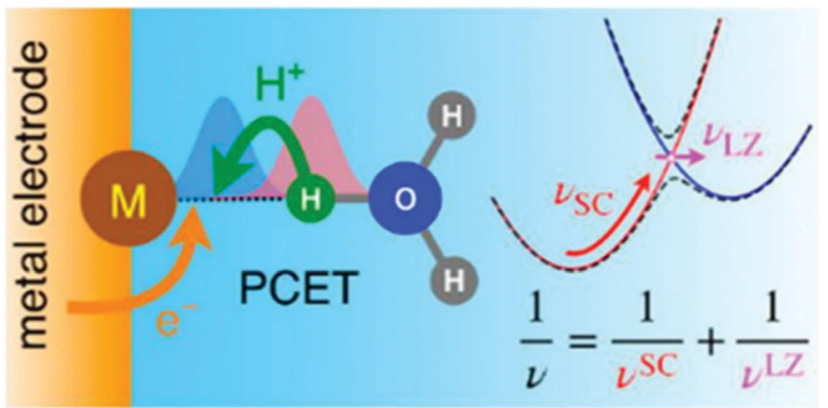

Fig. 10 Experimental and theoretical analysis for HER. (A) $\eta$ - $\log j_{\mathrm{k}}$ diagrams of the $\mathrm{HER}$ at Au electrode in $0.05 \mathrm{M} \mathrm{H}_{2} \mathrm{SO}_{4}$ in $\mathrm{H}_{2} \mathrm{O} / 0.05 \mathrm{M} \mathrm{D}_{2} \mathrm{SO}_{4}$ in $\mathrm{D}_{2} \mathrm{O}$. (B) Schematic illustration of the RDS for the HER at Au electrode in acidic condition $\left(\mathrm{H}_{3} \mathrm{O}^{+}+\mathrm{e}^{-}+{ }^{*} \rightarrow \mathrm{H}^{*}+\mathrm{H}_{2} \mathrm{O}\right)$. (C) Tafel plot (logarithm of current density versus potential) calculated by the theory and the equations in the reference for the reaction described in (B). ${ }^{94}$ The calculated data are shown as filled circles, and the lines represent linear fits to the calculated data points. The authors note here that in this case the potential (V vs. RHE) is identical to the overpotential (V). (D) Schematic illustration for the microscopic mechanism of PCET associated with vibrational nonadiabaticity and solvent dynamics. The panels A and B, and the related caption are reprinted from the ref. 47, Copyright (2020), with permission from Elsevier. The panels C and D are reprinted from the ref. 94, Copyright (2019), with permission from American Chemical Society.

been extensively considered at solid/gas interfaces by Freund and his co-workers. ${ }^{48,49,52,144-147}$ The other idea is to use the advantage of MOFs/COFs, where electronic structures can be controlled by selections and combinations of organic monomers and metal ions. ${ }^{100,119,132}$ The proof-of-concept was first shown for HER and ORR in 2017 (Fig. 11C and D). ${ }^{105}$ In this work, a model heterojunction electrode of the $2 \mathrm{D} \mathrm{COF} / \mathrm{Au}$ was used to investigate the effect of a $2 \mathrm{D} \mathrm{COF}$ as a porous-architecture to control electrocatalytic activity and showed that the additional thin COF layer is the key to improving activity or selectivity of the reactions. ${ }^{105}$ The identical concept was applied to a Au single crystal (111) surface and again confirmed that a thin COF layer can improve the HER activity. ${ }^{148}$ These advanced materials science efforts can contribute to understanding further details of microscopic mechanisms in quantum electrode processes together with other classes of well-defined modern designer materials, for instance molecular electrocatalysts (Fig. 11E and F). ${ }^{90,149-159}$

\section{Operando IR and Raman electro- chemical spectrometry for electrode processes at solid-liquid interfaces}

\subsection{Brief overview of present operando IR/Raman electrochemical spectrometry}

Operando observation of electrode-electrolyte interfaces by spectroscopic methods is attracting enormous interest because of its importance in modern electrochemistry. Infrared spectroscopy 

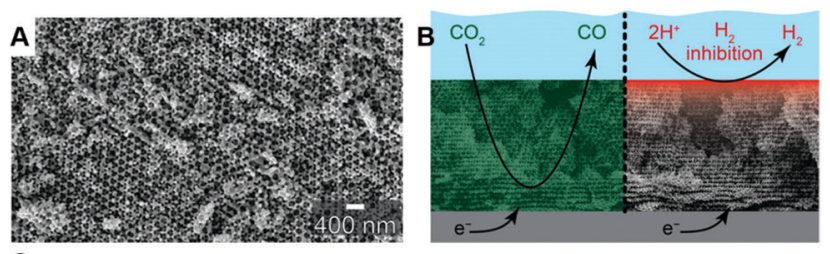

C : : : : N

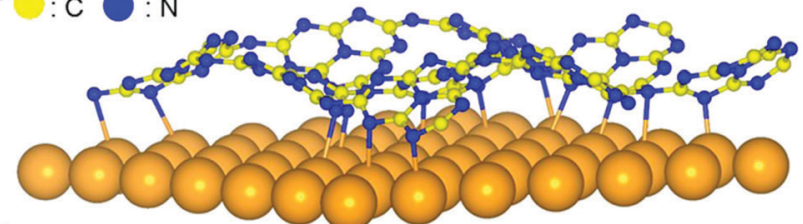

D

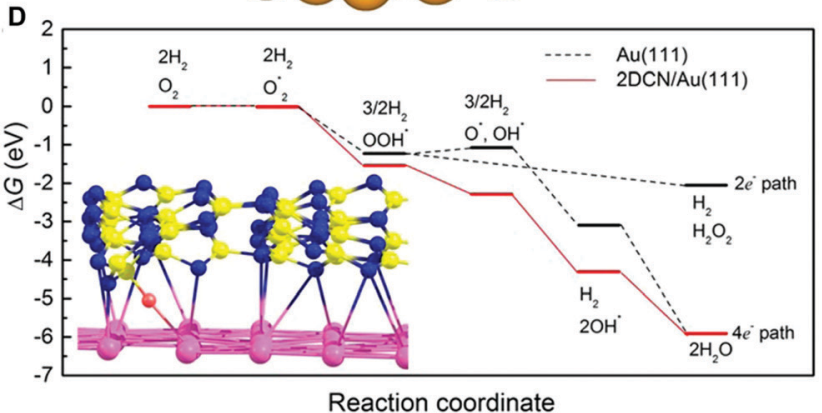

Reaction coordinate

E

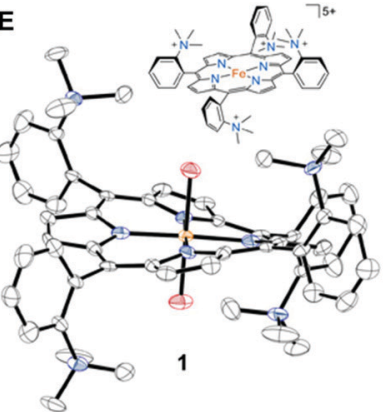

$\mathbf{F}$
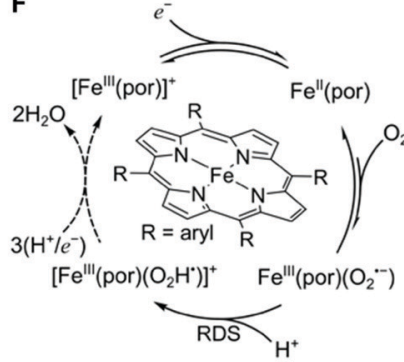

Fig. 11 Representative modern electrocatalysts in fundamental electrode process science. (A) Scanning electron microscope image of a precise inverse-opal $(\mathrm{IO}) \mathrm{Au}$ electrode and $(\mathrm{B})$ application of $\mathrm{IO}$ Au electrode to control of the electrochemical $\mathrm{CO}_{2}$-to- $\mathrm{CO}$ conversion efficiency by taking the advantage of functions due to porous structure. (C) Schematic illustration of 2D COF/Au heterojunction electrode and (D) its free-energy diagram (red line) showing the alternative reaction path to obtain a fourelectron transfer process which is not possible in Au electrode (black line). (E) Illustration of Fe-O-TMA of a model porphyrin-based molecular ORR electrocatalyst and the solid-state $\mathrm{X}$-ray crystal structure of [Fe-O-TMA. $2 \mathrm{H}_{2} \mathrm{O} J O T f_{5}(\mathrm{H}$ atoms and triflates omitted, thermal ellipsoids at 50\% probability). (F) ORR mechanism of Fe-porphyrin-based electrocatalysts. The panels A and B are reprinted from the ref. 111, Copyright (2015), with permission from American Chemical Society. The panels $C$ and $D$ are reprinted from the ref. 105, Copyright (2017), with permission from American Chemical Society. The panels E and F, and the corresponding captions are adopted from the ref. 157, Copyright (2020), with permission from American Association for the Advancement of Science.

and Raman spectroscopy are widely used to acquire information about various states of molecules on an electrode and electrocatalytic reaction pathways under applied potentials due to their simple set-up and high sensitivity. Application of infrared spectroscopy to electrochemistry has been developed rapidly with support by Fourier-transformed infrared spectroscopy
(FT-IR) since the 1980s. ${ }^{160,161}$ The mechanism of CO adsorption and alcohol oxidation on single-crystal metal electrodes has been intensively investigated by using Infrared reflection absorption spectroscopy (IRAS) measurements because they are able to detect a wide spectrum of adsorbed chemical species. ${ }^{162-165}$ Time-resolved spectroscopic techniques combined with IRAS measurement have been also developed for the kinetic study of the electrode processes. ${ }^{166}$ The adsorption dynamics of CO on Pt electrodes were observed at millisecond-scale. However, the timelag between the real electrode potential and the applied potential caused by the external reflection configuration for IRAS complicates the kinetic analysis. ${ }^{167}$ Since the 1990 s, technological improvements in equipment and software allow operando investigations with a higher sensitively and the capability for applications to a variety of different conditions. Surface-enhanced infrared absorption spectroscopy (SEIRAS) measurement by using a thin metal film electrode prepared on an attenuated-totalreflection (ATR) window has been developed by Osawa et al. ${ }^{168}$ The sensitivity of SEIRAS is higher by one digit than that of IRAS. Moreover, the internal reflection configuration for SEIRAS is compatible with time-resolved techniques. Time-resolved SEIRAS measurements have been applied to various electrode processes for elucidation of the kinetics at tens of milliseconds (rapid-scan interferometry) or submillisecond (step-scan interferometry) resolution. ${ }^{169-174}$ In recent years, the dynamics of elementary processes on electrocatalysts have been investigated by ultrafast time-resolved pump-probe IR and Raman spectroscopy at picosecond- or femtosecond-scales, which is recognized as an interesting approach. ${ }^{175-177}$ In addition to IR spectrometry, Raman spectroscopy has a long history too. As a highly sensitive method, surface-enhanced Raman spectroscopy (SERS) on a roughened substrate or nanoparticle of gold and silver was established from the 1970 s to the 1980 s. ${ }^{178}$ Furthermore, shell-isolated nanoparticle-enhanced Raman spectroscopy (SHINERS) based on silica coated gold nanoparticles was reported as an advanced approach for SERS to prevent degradation of the nanoparticles under the electrochemical environment and to utilize single crystal electrodes. ${ }^{179}$ Therefore, infrared spectroscopy and Raman spectroscopy are widely used to observe the electrode-electrolyte interface as complementary methods combined with standard electrochemical experiments to study the kinetics of electrode processes.

The investigation of the KIE associated with the ratedetermining step is valid for the elucidation of the PCET mechanisms (see Sections 3 and 5 for details). ${ }^{82,180}$ IR and Raman spectroscopy can identify isotopes, such as adsorbed H/D and OH/OD. For example, Pt-D stretching band $\left(\sim 1500 \mathrm{~cm}^{-1}\right)$ appears at lower frequency than that of $\mathrm{Pt}-\mathrm{H}$ stretching band $\left(\sim 2100 \mathrm{~cm}^{-1}\right)$. The frequency ratio can be estimated at $\sqrt{m_{\mathrm{D}} / m_{\mathrm{H}}}$ by harmonic oscillator approximation. Behm et al. revealed the potential-dependent KIE for the $\mathrm{C}-\mathrm{H}$ bond breaking associated with the rate-determining step during the electro-oxidation of formic acid on a Pt-film electrode using by SEIRAS. ${ }^{181}$ In other studies, the potential dependent KIEs for ethylene glycol electro-oxidation and $\mathrm{CO}_{2}$ electro-reduction have been investigated using by SEIRAS. ${ }^{182,183}$ The combined use 
of KIE method and time-resolved spectroscopic techniques can strongly support the other experiments and theories for the PCET reactions.

In recent years, spectroscopic methods have been applied to understand the mechanisms of electrocatalytic reactions, such as the hydrogen evolution reaction (HER) and oxygen reduction reaction (ORR). In particular, the development of highly active electrocatalysts for the ORR is important for fuel-cells and metal-air batteries, ${ }^{184}$ as IR and Raman spectroscopy can detect the intermediates and suggest the reaction pathway. ${ }^{161}$ However the high complexity of these reactions prevents a detailed understanding of the microscopic mechanisms (see Section 8 for details); therefore the reaction mechanism of HER, one of the simplest electrode processes, is still an open question. This Section presents the ongoing challenges for the investigation of the microscopic mechanisms of the HER and the ORR on electrified surfaces by using surface-electrochemicalspectroscopic approaches.

\subsection{Operando surface-electrochemical spectrometry for hydrogen evolution reaction}

The HER is a cathodic process for water electrolysis and also the reverse process of the hydrogen oxidation reaction (HOR) in the working reaction of fuel-cell. Three elementary steps are suggested for the HER. ${ }^{185-187}$ The first step is the adsorption of a hydrogen atom from the hydronium ion in the solution onto the electrode (Volmer step). The second step is the association and desorption reaction between the adsorbed hydrogen atom and the hydronium ion (Heyrovsky step). The other possible second step is the association and desorption reaction between two adsorbed hydrogen atoms (Tafel step). The kinetics of the HER has been widely studied by electrochemical analysis. For example, on the Pt electrode, the HER proceeds by the VolmerTafel process, and the rate-determining step is the Tafel step. ${ }^{185}$ However, recent studies proposed the surface-orientation dependence of the HER process on the Pt electrode, i.e. the Volmer-Tafel process proceeds on $\mathrm{Pt}(110)$, while the VolmerHeyrovsky process proceeds on $\mathrm{Pt}(100) .{ }^{185}$

To investigate the orientation-dependent-mechanism, operando infrared spectroscopy is used to acquire information on the HER mechanism on the Pt electrode by monitoring the adsorbed intermediate associated with a change of potential. The results of SEIRAS measurements indicated a band around $2100 \mathrm{~cm}^{-1}$ assigned to the $\mathrm{Pt}-\mathrm{H}$ stretching vibration adsorbed at atop sites (atop $\mathrm{H}$ ) on the Pt polycrystalline electrode in the HER potential range ( $<0.1 \mathrm{~V} v s$. RHE). ${ }^{188,189}$ The band intensity corresponding to the atop $\mathrm{H}$ increases in parallel with the HER current density at more negative potentials. These results indicated that the rate-determining step is the Tafel step. A dependence of the intermediate on the surface-orientation of the Pt has been investigated by using single-crystal electrodes. ${ }^{190}$ The band of the atop $\mathrm{H}$ was observed on Pt(110) and high index planes with (110) step structure. In contrast, the band around $1630 \mathrm{~cm}^{-1}$ assigned to the $\mathrm{Pt}-\mathrm{H}$ stretching vibration adsorbed at the asymmetric bridge site (bridge $\mathrm{H}$ ) was observed on $\mathrm{Pt}(100)$ and high index planes with (100) terrace or a step structure. On $\mathrm{Pt}(111)$, a high-resolution electron energy loss spectrometry (HR-EELS) and first-principles calculations suggest that a hydrogen atom adsorbs at the fcc hollow site (fcc H). ${ }^{191}$ Recent years, Sugino et al. suggested the coexistence of both atop $\mathrm{H}$ and fcc $\mathrm{H}$ on $\mathrm{Pt}(111)$ using by the random phase approximation for the correlation functional of DFT (see Section 7 for details). ${ }^{192}$ This insight is consistent with operando sum-frequency generation (SFG) spectroscopy. ${ }^{193}$ Unfortunately, IRAS bands originated in fcc $\mathrm{H}$ on $\mathrm{Pt}(111)$ have never been reported under electrochemical conditions because of the optical restriction for the lower frequency. Raman spectroscopy measurement may provide a breakthrough in the structure and kinetics of hydrogen atom adsorbed on Pt(111). The HER/HOR exchange current density on $\mathrm{Pt}(110)$ is higher than that of $\mathrm{Pt}(100)$ and Pt(111). ${ }^{185}$ These results suggest the HER kinetics may be enhanced through the atop $\mathrm{H}$.

\subsection{Operando surface-electrochemical spectrometry for oxygen reduction reaction}

The oxygen reduction reaction (ORR) occurs as two- or fourelectrons processes depending on $\mathrm{pH}$ and/or the specific electrocatalyst. For a long time, it was generally accepted based on experimental results that the four- and the two-electron processes, respectively, occur on $\mathrm{Pt}$ and $\mathrm{Au} .{ }^{194}$ In addition to experimental results, DFT calculations were used to compute the so-called volcano relationship, a diagram that correlates the ORR activity and the oxygen binding energy $\left(\Delta E_{\mathrm{O}}\right) \cdot{ }^{194}$ As this diagram is based on the previous approaches of Parsons based on the Sabatier principle, a better electrocatalyst has an optimal adsorption energy of a key intermediate in the corresponding reaction, i.e. a high enough adsorption energy to follow a favorable reaction path but not too high to release the product efficiently. In this computationally-calculated diagram, the $\operatorname{Pt}(111)$ surface was suggested to be located at the position to have the highest ORR activity among other typical precious metals for the four-electron process. On the other hand, the $\mathrm{Au}(111)$ surface has a higher $\Delta E_{\mathrm{O}}$ than Pt. The adsorbed oxygen atom on the $\mathrm{Au}(111)$ surface is unstable, and this instability was suggested to be the reason for the incapability of the $\mathrm{Au}(111)$ surface to transfer four protons and four electrons to the oxygen at this electrocatalyst under acidic conditions.

Aiming to obtain further insights of the ORR mechanism, infrared spectroscopy and Raman spectroscopy have been applied to the ORR mechanism on the $\mathrm{Au}$ and Pt electrode through the adsorbed intermediate. By using SEIRAS the intermediate during the ORR on Au polycrystalline electrode was observed in both alkaline and acidic solutions. ${ }^{195}$ The asymmetric bending mode of the adsorbed $\mathrm{OOH}\left(\mathrm{OOH}_{\mathrm{ad}}\right)$, which is suggested as an intermediate during the four-electron process, appeared between 0.1 and $-0.6 \mathrm{~V}$ vs. $\mathrm{Ag} / \mathrm{AgCl}$ in an $\mathrm{O}_{2}$-saturated alkaline solution. The previous studies of the ORR on Au single-crystal electrodes suggested that the four-electron process occurs only on the $\mathrm{Au}(100)$ surface in alkaline solutions. ${ }^{196,197}$ In contrast, the band intensity of $\mathrm{OOH}_{\mathrm{ad}}$ in acidic solutions is weaker than that in alkaline solutions. These studies suggested that the key intermediate for the four-electron process of $\mathrm{OOH}_{\mathrm{ad}}$ is unlikely to be the main product, and therefore the two-electron process occurs on the Au surface in acidic 
solutions. In another study, the two bands assigned to the $\mathrm{O}-\mathrm{O}$ stretching mode of adsorbed $\mathrm{O}_{2}{ }^{-}\left(\mathrm{O}_{2}{ }^{-}\right.$ad $)$and the $\mathrm{O}-\mathrm{O}$ stretching mode of $\mathrm{HOOH}\left(\mathrm{HOOH}_{\mathrm{ad}}\right)$ on the $\mathrm{Au}$ roughened surface were observed in alkaline solutions below $0.85 \mathrm{~V} v s$. RHE by a SERS measurement. ${ }^{198}$ This observation supports the fourelectron process on $\mathrm{Au}$ electrodes in alkaline media. On the other hand, the band intensity of $\mathrm{O}_{2}{ }^{-}$ad was weak and $\mathrm{HOOH}_{\mathrm{ad}}$ was not observed in acidic solution. This fact suggests that the one-electron process mainly occurs as the RDS, and some oxygen atoms are reduced afterwards through the two-electron process via $\mathrm{O}_{2}{ }^{-}$ad in acidic solutions. Furthermore, another study based on a SERS measurement indicated that the fourelectron process occurs on the Au surface modified with Bi even in acidic solution. ${ }^{199}$

On Pt electrodes, the band corresponding to the $\mathrm{O}-\mathrm{O}$ stretching mode of $\mathrm{O}_{2}{ }^{-}$ad was observed by a SEIRA measurement in alkaline solutions, and this report claimed that the $\mathrm{HOOH}_{\mathrm{ad}}$ is related to the RDS of the four-electron process. ${ }^{200}$ In acidic solutions, attenuated total reflection infrared (ATR-IR) spectrometry was used to observe the ORR intermediates on $\mathrm{Pt} / \mathrm{C}$ nanoparticles. ${ }^{201}$ Fig. 12A shows the ATR-IR spectra on

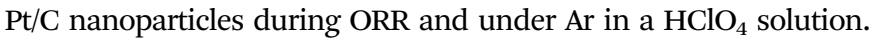
In this study, three intermediates were observed during the ORR, and a possible mechanism was proposed based on this observation (Fig. 12B). The observation of the $\mathrm{O}-\mathrm{O}$ stretching mode of $\mathrm{OOH}_{\mathrm{ad}}$ and the $\mathrm{OOH}$ bending mode of $\mathrm{HOOH}_{\mathrm{ad}}$ indicates that the $\mathrm{O}-\mathrm{O}$ bond breaking occurs after the protonation of the $\mathrm{OOH}_{\mathrm{ad}}$, and therefore the ORR mainly proceeds through the associative pathway. However, the band of $\mathrm{OOH}_{\mathrm{ad}}$ appears at $0.9 \mathrm{~V}$ (RHE), whereas $\mathrm{HOOH}_{\mathrm{ad}}$ appears only below $0.7 \mathrm{~V}$. This suggests that the
ORR mainly proceeds through the dissociative pathway at higher potentials. The dependence of the ORR mechanism on the surfaceorientation has been investigated using SHINERS measurement. The ORR occurs via $\mathrm{OOH}_{\mathrm{ad}}$ on $\mathrm{Pt}(111)$, whereas it occurs via $\mathrm{OH}_{\mathrm{ad}}$ on $\mathrm{Pt}(100)$ and $\mathrm{Pt}(110) .{ }^{202}$ SHINERS was used to observe the $\mathrm{O}-\mathrm{O}$ stretching mode of $\mathrm{OOH}_{\mathrm{ad}}$ and the Pt-OH bending mode of $\mathrm{OH}_{\mathrm{ad}}$ on high-index planes of $\mathrm{Pt}^{203}$

In addition to the mechanism, the activity of the ORR on Pt is also affected by surface adsorbed species such as specificallyadsorbed anions and adsorbed hydroxide $\left(\mathrm{OH}_{\mathrm{ad}}\right)$. Sulfate and bisulfate anions specifically adsorb on the Pt(111) surface, and therefore the ORR activity in $\mathrm{H}_{2} \mathrm{SO}_{4}$ solutions is much lower than that in $\mathrm{HClO}_{4}$ solutions. ${ }^{204-206}$ An IRAS measurement was used to investigate the $\mathrm{SO}_{3}$ symmetric stretching mode of adsorbed (bi)sulfate anion at the three-fold site of the Pt(111) surface via three oxygen atoms. ${ }^{207,208}$ Perfluorosulfonic acids (PFSA) such as Nafion used for polymer electrolytes also specifically adsorb on the Pt(111) surface and decrease the ORR activity. ${ }^{209-211}$ The IRAS and SEIRAS measurements suggested that the sulfonate group in PFSA adsorbs on Pt via one or two oxygen atoms, and the ether group also interacts with the surface. ${ }^{211,212}$ The adsorbed species formed during the surface oxidation such as $\mathrm{OH}_{\mathrm{ad}}$ is a key factor in the ORR activity as well as the specifically-adsorbed electrolyte species. The equation of $\mathrm{OH}_{\mathrm{ad}}$ formation is as follows:

$$
\mathrm{Pt}+\mathrm{H}_{2} \mathrm{O} \rightarrow \mathrm{Pt}-\mathrm{OH}+\mathrm{H}^{+}+\mathrm{e}^{-}
$$

The ORR activity of Pt low-index planes at $0.9 \mathrm{~V}$ (RHE) increases by following the order of $\mathrm{Pt}(110)>\mathrm{Pt}(111)>\mathrm{Pt}(100)$ in $\mathrm{HClO}_{4}{ }^{213}$
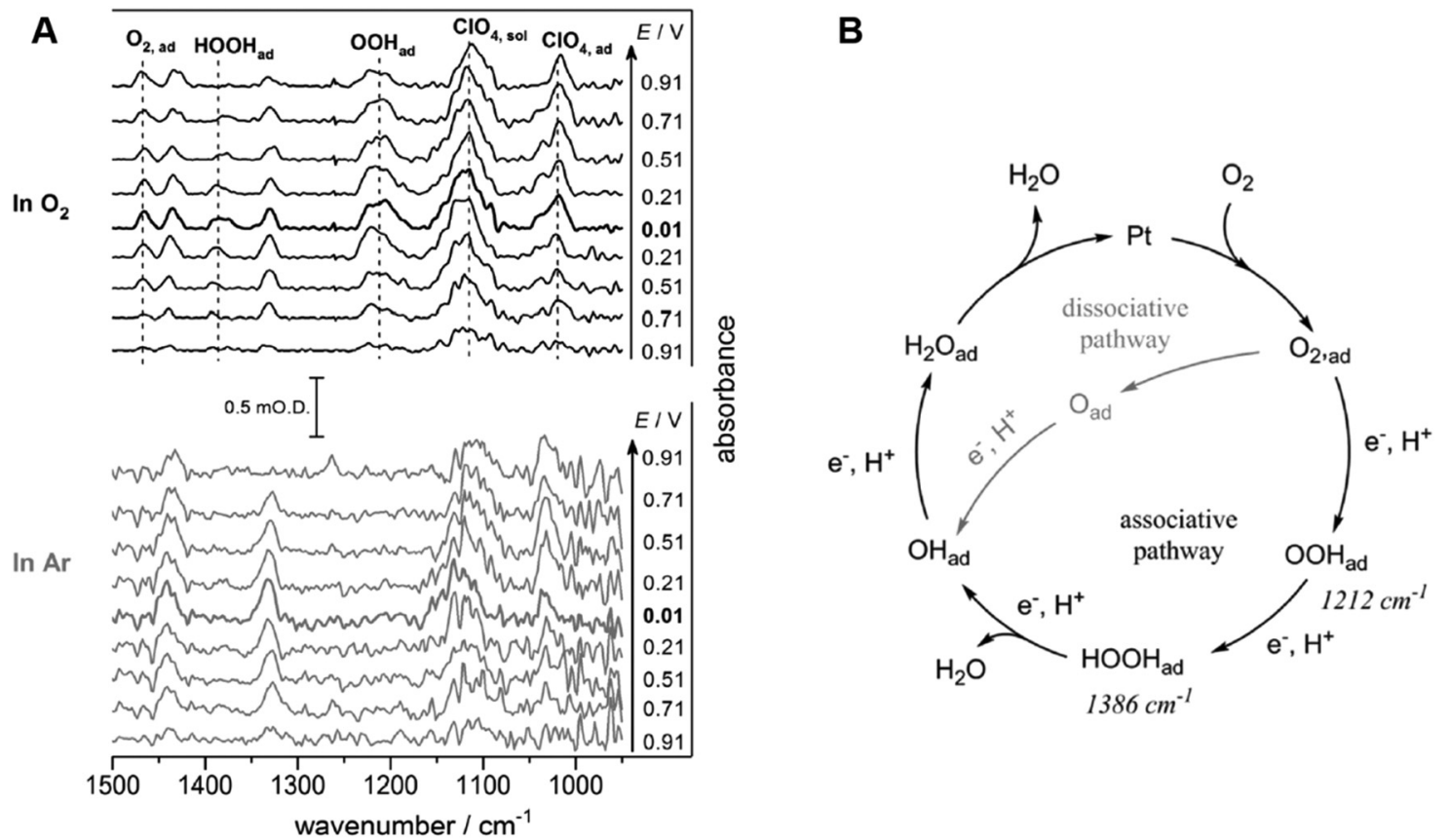

Fig. 12 (A) ATR-IR spectra on Pt/C nanoparticle during ORR and under Ar in $0.1 \mathrm{M} \mathrm{HClO}_{4}$. The reference spectra are obtained at $1.01 \mathrm{~V}$ vs. SHE. (B) Proposed mechanism of ORR at a Pt/C nanoparticle catalyst in $0.1 \mathrm{M} \mathrm{HClO}_{4}$. The figure and caption were adopted from the reference with permission from the ref. 201. Copyright (2018) Wiley-VCH. 
The band assigned to the $\mathrm{Pt}-\mathrm{OH}$ bending vibration band was observed by an IRAS measurement, and this band intensity at $0.9 \mathrm{~V}$ increased by following the order of $\operatorname{Pt}(100)>\operatorname{Pt}(111)>$ Pt(110). ${ }^{214}$ Similarly, there was an inverse relationship between the band intensity and the ORR activity on high-index planes of $\mathrm{Pt}^{215}$ Based on the these investigations, the stability of $\mathrm{OH}_{\mathrm{ad}}$ was suggested to be dependent on the surface orientation of the electrocatalysts and therefore may determine the ORR activity as a similar feature is well-known in the field of solid-gas catalysis in ultrahigh vacuum (UHV) conditions. ${ }^{216}$ Not only surface orientation but also the $\mathrm{OH}_{\mathrm{ad}}$ formation and the ORR activity of Pt can depend on electrolyte cations. At the electrode-electrolyte interface, electrolyte cations are hydrated and behave as non-specifically adsorbed species but weakly interact with Pt surfaces through hydrogen bonds and electrostatic interactions, i.e., so-called non-covalent interactions. In alkaline solutions, the ORR activity of $\mathrm{Pt}(111)$ is related to electrolyte cations as follows: $\mathrm{Li}^{+}<\mathrm{Na}^{+}<\mathrm{K}^{+}<\mathrm{Cs}^{+}{ }^{217}$ The Pt-OH band in LiOH containing solution on $\mathrm{Pt}(111)$ was observed by an IRAS measurement, whereas no bands were observed in $\mathrm{CsOH}^{218}$ These results suggested that $\mathrm{OH}_{\mathrm{ad}}$ is a blocking species toward the ORR, and the formation of $\mathrm{OH}_{\mathrm{ad}}$ is dependent on the electrolyte cation. The hydration water around hydrophilic cations such as $\mathrm{Li}^{+}$is coordinated with the dipole moment pointed outward, and the hydrogen atoms of the hydration water can link with the outer oxygen species. ${ }^{219}$ Hydrated $\mathrm{Li}^{+}$has a stronger non-covalent interaction with the $\mathrm{Pt}(111)$ surface to stabilize $\mathrm{OH}_{\mathrm{ad}}$ than hydrated $\mathrm{Cs}^{+}$, and therefore the ORR activity in LiOH solutions is lower than that in $\mathrm{CsOH}$ solutions.

In addition to the above cations based on the alkaline metals, recent studies showed that organic cations, i.e., quaternary ammonium cations with long alkyl chains, enhanced the ORR activity of $\mathrm{Pt}^{220,221}$ The ORR activity of $\mathrm{Pt}(111)$ in $\mathrm{HClO}_{4}$ containing tetrahexylammonium cation $\left(\mathrm{THA}^{+}\right)$at $0.9 \mathrm{~V}$ is eight times higher than that without this organic cation. Fig. 13A shows the IRA spectra of $\mathrm{Pt}(111)$ and $\mathrm{THA}^{+}$modified $\mathrm{Pt}(111)$ surfaces in acidic solution. In this observation, the $\mathrm{Pt}-\mathrm{OH}$ bending mode around $1050 \mathrm{~cm}^{-1}$ was observed above $0.6 \mathrm{~V}$, which suggests that the addition of $\mathrm{THA}^{+}$inhibits the $\mathrm{OH}$ adsorption on the $\mathrm{Pt}(111)$ surface. Fig. 13B shows the potential dependence of the charge density of Pt surface oxidation estimated from cyclic voltammetry studies and the band intensity of $\mathrm{Pt}-\mathrm{OH} .^{221}$ The onset potential of $\mathrm{OH}$ adsorption shifts positively and the band intensity of $\mathrm{Pt}-\mathrm{OH}$ decreases due to the presence of $\mathrm{THA}^{+}$. These results are consistent with the charge density of $\mathrm{Pt}$ surface oxidation and suggests that $\mathrm{THA}^{+}$has some effects of destabilizing and decreasing $\mathrm{OH}_{\mathrm{ad}}$ on the $\mathrm{Pt}(111)$ surface. The positive- and negative-going bands around 1650 and $1610 \mathrm{~cm}^{-1}$ are assigned to the $\mathrm{HOH}$ bending mode of non-adsorbed and adsorbed hydrogen-bonded water, respectively. Similar multiple bands appeared around $1500 \mathrm{~cm}^{-1}$ on $\mathrm{THA}^{+}$-modified $\mathrm{Pt}(111)$. These bands can be assigned to the $\mathrm{HOH}$ bending mode of nonadsorbed and adsorbed water monomers. The adsorbed water monomer has been observed under UHV conditions. ${ }^{222}$ However, at the electrode-electrolyte interface, the water molecules are cross-linked via hydrogen bonding, and therefore the adsorbed water monomer is unstable. Unlike the hydration water shell around hydrophilic cations, the shell around hydrophobic cations such as $\mathrm{THA}^{+}$exhibits restricted coordination with the outer oxygen species because of the complete hydrogenbonding network within the shell. ${ }^{219}$ This specific hydration structure may stabilize the adsorbed water monomer. The enhancement effect for the ORR activity due to $\mathrm{THA}^{+}$did not appear on $\mathrm{Pt}(100)$ and $\mathrm{Pt}(110)$. The hydrogen-bonded co-adsorption layer of $\mathrm{OH}_{\mathrm{ad}}$ and water forms on the $\mathrm{Pt}(111)$ surface because the symmetry and the hydrogen bonding distance fit with the $\mathrm{Pt}(111)$ lattice. Hydrophobic species destabilize the hydrogen bonding network and form an efficient interface for the ORR. On Pt(100) and $\operatorname{Pt}(110)$, the hydrogen bonding network is weak due to the lattice mismatch. $\mathrm{THA}^{+}$specifically destabilizes the hydrogen bonding network and decreases the coverage of the $\mathrm{OH}_{\mathrm{ad}}$ species on the Pt(111) surface. As a result, an efficient electrode-electrolyte interface for the ORR is formed. The ORR kinetics cannot be explained sufficiently by the thermodynamics of adsorbates. The above cations and water molecule may influence the quantum processes, such as proton and electron transfer. Kinetic studies at
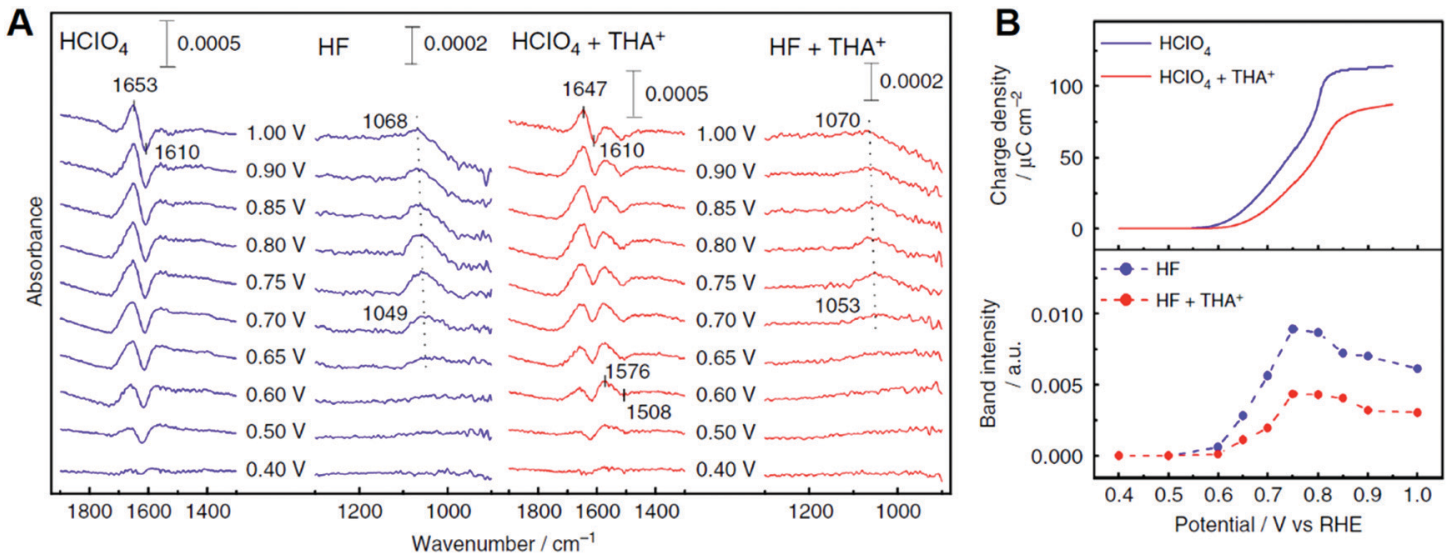

Fig. 13 (A) Infrared reflection absorption spectra on Pt(111) and Pt(111) modified with tetrahexylammonium cation (THA ${ }^{+}$) in $0.1 \mathrm{M}^{2}$ acidic solutions saturated with Ar. The reference spectra are obtained at $0.30 \mathrm{~V}$ vs. RHE. The potentials are stepped in the positive direction. (B) Potential dependence of the charge density of Pt oxidation and the band intensity of Pt-OH. The figure and caption were adopted from the ref. 221. 
the characteristic electrode/electrolyte interface using by spectroscopic measurements combined the KIE method elucidate the microscopic mechanism for the efficient ORR.

Recent years, non-Pt based catalysts such as iron and cobalt porphyrin complexes for the ORR have been widely investigated. $^{223}$ Living things convert the energy from the ORR efficiently using by these complex catalysts. Cytochrome is an heme protein containing iron porphyrin complex, which acts as the ORR catalyst and the proton pump. ${ }^{24}$ The PCET mechanisms in the ORR by various types of cytochrome have been investigated using by IR and Raman spectroscopy. ${ }^{225,226}$ Naumann et al. found the potential- and time-dependent band intensity assigned to $\mathrm{C}-\mathrm{C}$ stretching mode of the porphyrine ring using by time-resolved SERS measurement. ${ }^{227}$ The band intensity is correlated to the transition from the reduced to the oxidized state of cytochrome via heterogeneous electron transfer. The rate constants of the electron transfer was estimated at $k_{\mathrm{ox}}=46 \pm 7 \mathrm{~s}^{-1}$ and $k_{\mathrm{red}}=84 \pm 20 \mathrm{~s}^{-1}$. The same group also estimated the rate constant from the band intensity assigned to the amide region using by time-resolved SEIRAS measurement. ${ }^{228,229}$ Weidinger et al. revealed the KIE for the electron transfer of iron porphyrin complexes from the band intensity assigned to the $\mathrm{C}=\mathrm{O}$ stretching mode of the protonated carboxylic acid using by the SERS and SEIRAS measurements. ${ }^{230}$ The heterogeneous electron transfer of the porphyrin complex is strongly modulated by the protonation state of the acidic group via hydrogen bond interactions.

\subsection{Outlooks for the future of operando surface- electrochemical spectrometry}

Operando observations using infrared spectroscopy and Raman spectroscopy are useful to elucidate electrocatalytic processes and molecular structures at electrode-electrolyte interfaces. The spectroscopic study combined with time-resolved techniques and the KIE method will strongly support the microscopic kinetics of the HER and the ORR. In addition to the HER and the ORR, the photocatalytic hydrogen production and the $\mathrm{CO}_{2}$ electroreduction are also interesting subjects for the spectroscopic study. ${ }^{231-234}$ Non-linear spectroscopy methods such as sum-frequency generation (SFG) spectroscopy and stimulated Raman scattering (SRS) can be applied to analyze the dynamics of electrode processes on the pico or femtosecond timescale..$^{235-239}$ Single-molecule spectroscopy and imaging techniques such as tip-enhanced Raman spectroscopy (TERS) will enable monitoring of electrochemical reactions with nanoscale resolution. ${ }^{240}$ These approaches are expected to provide further detailed information on complicated phenomena at electrified solid-liquid interfaces, such as elucidating the role of quantum effects.

\section{Heterogeneous PCET theory applied to the Volmer reaction}

\subsection{Basics of general PCET theory}

As discussed above, many electrochemical processes involve PCET. A general theoretical formulation for PCET that treats the active electrons and transferring protons quantum mechanically and includes the reorganization of the solvent environment, as well as the proton donor-acceptor motion, has been developed. ${ }^{241-244}$ In the context of Fig. $4,{ }^{67}$ this theoretical formulation covers the entire right branch, in which the electrons and protons are treated quantum mechanically. If the process is fully electronically and vibrationally adiabatic, where the electrons and protons respond instantaneously to the other nuclei, the transition state theory formulation is often applicable (case A in Fig. 4). If the process is vibrationally or vibronically nonadiabatic, in the absence of this instantaneous response, the Fermi golden rule nonadiabatic formulation is often applicable (cases B and C in Fig. 4), ${ }^{242,243}$ although the vibronic coupling has a different form in the electronically adiabatic and nonadiabatic regimes. ${ }^{243,245,246}$ In the more complex case of slow solvent dynamics, the rate constant expressions must also account for the solvent-controlled regime. ${ }^{247-250}$ This section will discuss the rate constants for all of these limits as well as the intermediate regimes, focusing primarily on electrochemical PCET.

\subsection{Concepts of heterogeneous electrochemical PCET theory}

Electrochemical PCET can be divided into homogeneous and heterogeneous processes (Fig. 14). In homogeneous electrochemical PCET, the electrode serves as a reservoir for donating and accepting electrons but is not involved in the chemical bond breaking and forming. In this case, the electron transfers between the solvated redox molecule and the electrode, and the proton transfer reaction occurs within the molecular complex. Homogeneous electrochemical PCET is described in detail elsewhere ${ }^{251-254}$ and will not be discussed further herein. In heterogeneous electrochemical PCET, the electrode participates in the chemical bond breaking and forming process. In the prototypical Volmer reaction, the proton is transferred from the molecular system, which could be specifically adsorbed on the surface, to the electrode, forming a metalhydrogen covalent bond. The remainder of this section will focus on heterogeneous electrochemical PCET in the context of the Volmer reaction.

In the acidic Volmer reaction, a proton is transferred from an acid, $\mathrm{AH}^{+}$, to a metal surface $\mathrm{M}$, forming the conjugate base A and a metal-hydrogen ( $\mathrm{MH}$ ) bond via an electron from the electrode:

$$
\mathrm{M}+\mathrm{AH}^{+}+\mathrm{e}^{-} \rightleftharpoons \mathrm{MH}+\mathrm{A} .
$$

This reaction can be described in terms of a diabatic electronic state for the reactant, corresponding to the $\mathrm{AH}^{+}$covalent bond, and a set of diabatic electronic states for the product, corresponding to the MH covalent bond associated with the continuum of energy levels in the metal electrode. ${ }^{255}$ The free energies of these diabatic electronic states depend on the proton coordinate $r$, a collective solvent coordinate $X$, and the distance of the proton-donating acid from the electrode $R$ (i.e., the proton donor-acceptor distance). ${ }^{255}$ Quantization of the proton produces a set of electron-proton vibronic states for the reactant and the product with free energies that depend on 


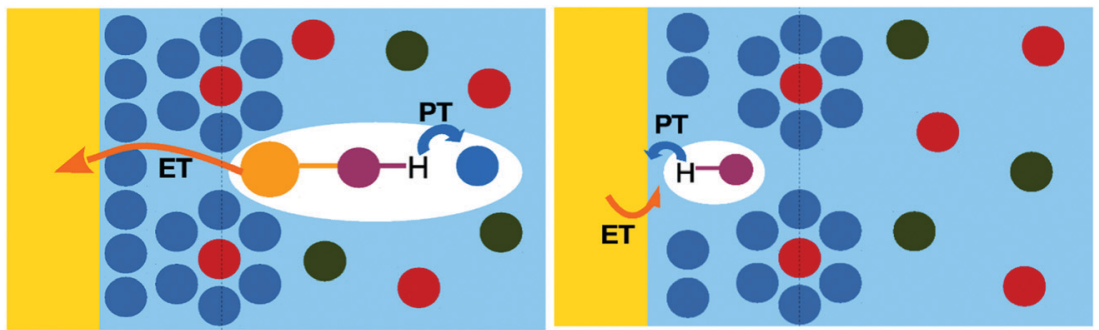

Fig. 14 Schematic depiction of homogeneous electrochemical PCET (left) and heterogeneous electrochemical PCET (right). In homogeneous PCET, the electrode serves as a reservoir for electrons but is not directly involved in the chemistry; the electron transfers between the molecule and the electrode, and the proton transfers within the molecular complex. In heterogeneous PCET, the electrode participates in the chemistry; in the Volmer reaction, the proton transfers from the molecule to the electrode, forming a metal-hydrogen bond.

$X$ and $R$. For a fixed distance $R$, reorganization of the solvent leads to an intersection between a pair of reactant and product vibronic free energy surfaces. In the vibronically nonadiabatic regime, a nonadiabatic transition can occur at the intersection point with a probability depending on the square of the vibronic coupling between the reactant and product vibronic states. The total rate constant is obtained by summing over transitions between all reactant and product vibronic states, weighting the reactant states by their Boltzmann populations. ${ }^{242,243,251}$ In the vibronically adiabatic regime, the reaction occurs on the vibronic ground state free energy surface, which smoothly transitions from the reactant to the product. $^{255}$

In both regimes, the resulting rate constant $k(R, E)$ depends on the distance $R$ from the electrode and the applied electrode potential $E$. The current density is obtained by averaging this rate constant over the distance $R$, weighting by the concentration $c_{\mathrm{HA}^{+}}(R)$ of the acid:

$$
j(E)=F \int \mathrm{d} R c_{\mathrm{HA}^{+}}(R) k(R, E)
$$

where $F$ is Faraday's constant. The concentration $c_{\mathrm{HA}^{+}}(R)$ can be modeled as a Gaussian distribution function centered at the equilibrium distance for a specifically adsorbed species. ${ }^{93}$ The form of the rate constant $k(R, E)$ for the Volmer reaction depends on the physical properties of the system, including the magnitude of the electronic coupling and the solvent relaxation timescale. ${ }^{94}$

The form of the cathodic rate constant in the vibronically nonadiabatic regime for heterogeneous electrochemical PCET can be derived using Fermi's golden rule, leading to the following expression: ${ }^{93,242,243,251}$

$$
\begin{aligned}
k(R, E)= & \frac{\rho_{\mathrm{F}}}{\hbar} \sqrt{\frac{\pi}{\lambda k_{\mathrm{B}} T}} \\
& \times \sum_{\mu, \nu} P_{\mu}\left|V_{\mu \nu}(R)\right|^{2} \int \mathrm{d} \varepsilon f(\varepsilon) \exp \left[-\frac{\left(\Delta G_{\mu \nu}(R, E, \varepsilon)+\lambda\right)^{2}}{4 \lambda k_{\mathrm{B}} T}\right] .
\end{aligned}
$$

Here the electrode potential $E$ is measured relative to a reference potential $E_{\mathrm{Ref}}, \rho_{\mathrm{F}}$ is the electrode density of states at the Fermi level, and $f(\varepsilon)$ is the Fermi distribution function for the electronic states in the electrode. The double summation is over all pairs of reactant $(\mu)$ and product $(\nu)$ electron-proton vibronic states, $P_{\mu}$ is the Boltzmann population of the reactant vibronic state $\mu, V_{\mu \nu}(R)$ is the vibronic coupling between states $\mu$ and $\nu, \Delta G_{\mu \nu}(R, E, \varepsilon)$ is the reaction free energy for the transition between the states $\mu$ and $\nu$, and $\lambda$ is the total reorganization energy. The reaction free energy can be expressed as ${ }^{93}$

$$
\Delta G_{\mu \nu}(R, E, \varepsilon)=\Delta G^{\circ}+\Delta \varepsilon_{\mu \nu}-\varepsilon+e\left(E+E_{\mathrm{Ref}}\right)-e \psi(R, E)-\Delta W(R)
$$

where $\Delta G^{\circ}$ is the reaction free energy in bulk solution, $\Delta \varepsilon_{\mu \nu}$ is the difference between the proton vibrational energy levels relative to the minima of their respective potentials for the product vibronic state $\nu$ and the reactant vibronic state $\mu, \psi(R, E)$ is the electrostatic potential relative to bulk solution, and $\Delta W(R)$ is the non-electrostatic work term.

The electrostatic potential $\psi(R, E)$ can be determined using a suitable model for the electrical double layer (EDL). A practical approach that includes the key physical properties of the EDL is a recently developed extended Guoy-Chapman-Stern model. ${ }^{93,256-259}$ This extended model describes the electrodesolvent interface with three regions that each have different dielectric constants. ${ }^{93}$ The inner Helmholtz layer is assigned a relatively small dielectric constant to describe the constrained mobility of the first layer of solvent molecules, the diffuse layer is assigned the solvent dielectric constant, and the intervening outer Helmholtz layer is assigned a variable dielectric constant that depends on the applied electrode potential. ${ }^{259}$ These types of dielectric continuum models provide qualitatively reasonable descriptions of the EDL but do not describe the detailed behavior of the electrostatic potential at the interface. The electrostatic potential at a gold electrode surface has been shown to be inhomogeneous and to depend strongly on the applied electrode potential. ${ }^{260}$ Explicit solvent molecules and ions must be included to capture the detailed interfacial behavior. Nevertheless, the simple dielectric continuum models that incorporate the essential physical properties of the EDL are useful for computing heterogeneous PCET rate constants.

The form of the vibronic coupling $V_{\mu \nu}(R)$ depends on the degree of electron-proton nonadiabaticity. ${ }^{245,246}$ Within the vibronically nonadiabatic regime, the electronically adiabatic and nonadiabatic limits correspond to cases B and C, respectively, in Fig. 4. In the electronically nonadiabatic limit, $V_{\mu \nu}(R)$ is the 
product of the electronic coupling and the overlap integral between the reactant and product proton vibrational wavefunctions associated with vibronic states $\mu$ and $\nu$. In the electronically adiabatic limit, $V_{\mu \nu}$ is half the tunneling splitting $\Delta_{\mu \nu}$ between the delocalized proton vibrational states computed for the electronically adiabatic proton potential curve associated with alignment of the energies of vibronic states $\mu$ and $\nu$. The general expression for the vibronic coupling spanning both regimes is ${ }^{245,246}$

$$
V_{\mu \nu}=\kappa_{\mu \nu} \frac{\Delta_{\mu \nu}}{2}
$$

where $\kappa_{\mu \nu} \ll 1$ in the electronically nonadiabatic regime and $\kappa_{\mu \nu} \approx 1$ in the electronically adiabatic regime. More details about computing this prefactor and the vibronic coupling given two diabatic electronic proton potential energy curves are provided elsewhere. ${ }^{93,246}$

\subsection{Application of heterogeneous electrochemical PCET theory to uncover microscopic mechanism of Volmer reaction}

This vibronically nonadiabatic approach was used to investigate the Volmer reaction corresponding to proton discharge from triethylammonium $\left(\mathrm{TEAH}^{+}\right)$to a gold surface in acetonitrile (Fig. 15). ${ }^{93}$ Experimental studies of this process revealed significantly different Tafel slopes for $\mathrm{TEAH}^{+}$and its deuterated counterpart, $\mathrm{TEAD}^{+}$, leading to a potential-dependent kinetic isotope effect (KIE) for the hydrogen evolution reaction. ${ }^{95}$ A theoretical analysis of this process suggested that it is vibronically nonadiabatic, enabling the use of the rate constant in eqn (6), and is predominantly but not fully electronically adiabatic, necessitating the use of the general expression for the vibronic coupling given in eqn (8) to describe the intermediate regime. ${ }^{93}$ Application of this theoretical formulation in conjunction with a physically reasonable model reproduced the experimental observations (Fig. 15). ${ }^{93,95}$ The physical explanation for the potential-dependent KIE emerging from this study was that different pairs of reactant and product vibronic states dominate for hydrogen and deuterium with relative contributions that depend on the applied potential. In particular, the smaller energy splittings between the vibrational states for deuterium result in greater contributions from the excited reactant vibronic states for $\mathrm{TEAD}^{+}$compared to $\mathrm{TEAH}^{+}{ }^{93}$ This application highlights the importance of nuclear quantum effects, nonadiabaticity, and excited vibronic states in heterogeneous PCET. It also provides an illustration of how PCET reactions proceeding by the same fundamental mechanism can exhibit different Tafel slopes.

For the Volmer reaction in aqueous solution, the proton is typically presumed to be donated directly from $\mathrm{H}_{3} \mathrm{O}^{+}$, which is
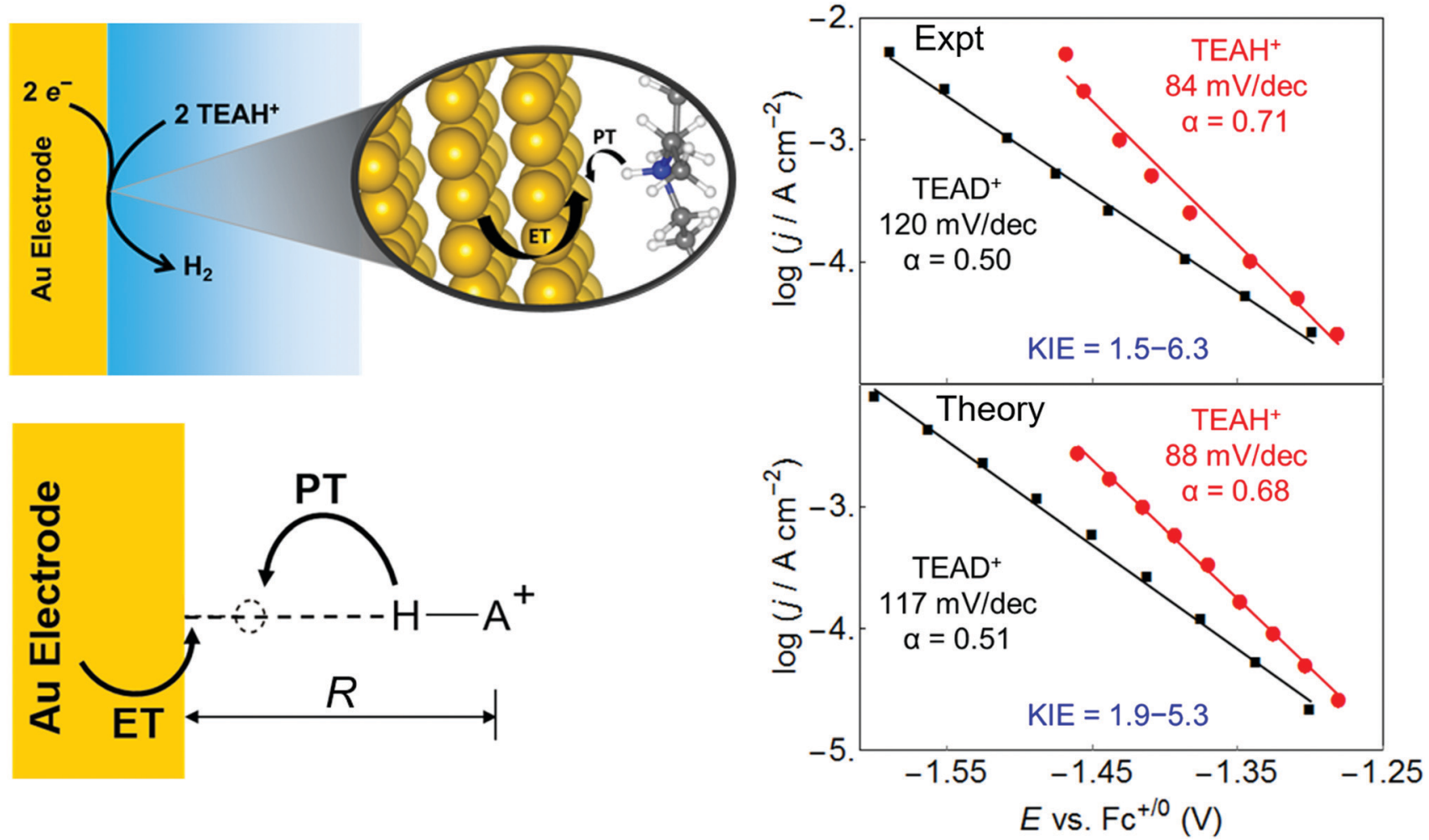

Evs. Fc (V)

Fig. 15 Left: Schematic depiction of the PCET reaction associated with proton discharge from $\mathrm{TEAH}^{+}$to a gold electrode, corresponding to the Volmer reaction that is a key step in the hydrogen evolution reaction. Right: Tafel plots, which reflect the current density versus the applied potential, obtained from experimental data (upper panel) and theoretical calculations (lower panel) for the PCET reaction associated with proton discharge from TEAH ${ }^{+}$(red circles) or TEAD ${ }^{+}$(black, squares) to a gold electrode in acetonitrile. The reported Tafel slopes and electrochemical transfer coefficients, $\alpha$, are obtained from linear fits to the data points shown. The smallest and largest KIEs reported are the ratios between the TEAH ${ }^{+}$and TEAD ${ }^{+}$linear fits at $-1.30 \mathrm{~V}$ and -1.46 V, respectively. Figures adapted with permission from ref. 93. Copyright 2018 American Chemical Society. 


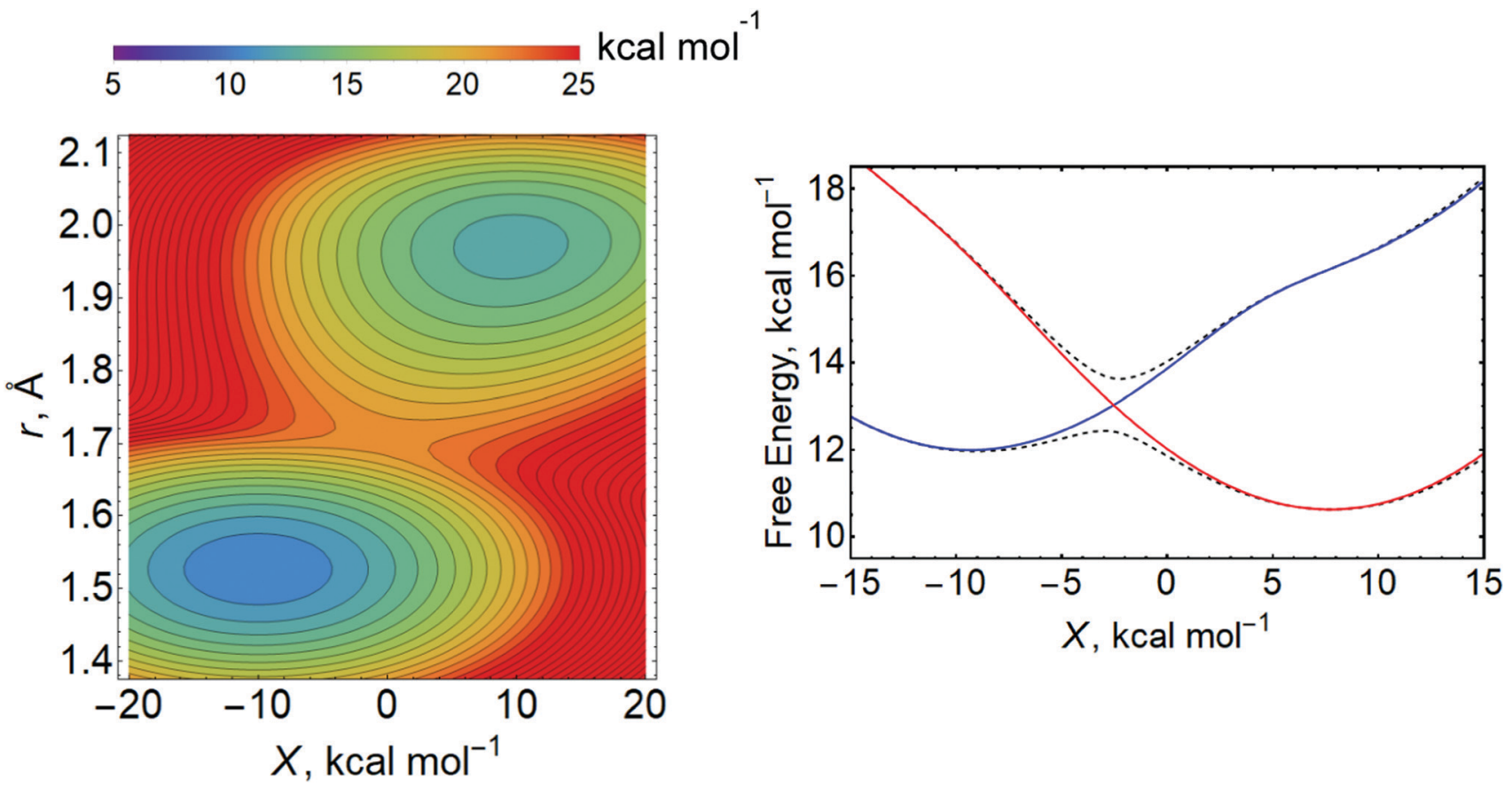

Fig. 16 Left: Example of two-dimensional free energy surface as function of the proton coordinate $r$ and the collective solvent coordinate $X$ computed at a fixed distance $R$ between the acid and the electrode surface. Right: Example of free energy surfaces for the lowest two adiabatic vibronic states (dashed black lines) and associated diabatic vibronic states (solid lines, reactant state in red, product state in blue) as a function of the collective solvent coordinate $X$ at a fixed $R$ obtained by quantization of the proton coordinate. Left figure adapted with permission from ref. 255 . Copyright 2019 American Chemical Society. Right figure adapted with permission from ref. 94. Copyright 2019 American Chemical Society.

hydrogen bonded to other water molecules in some type of protonated water cluster. Because the proton-donating acid is located near or adsorbed to the surface, leading to relatively strong electronic coupling and short proton donor-acceptor distances, the aqueous Volmer reaction is often assumed to be fully adiabatic (i.e., in the vibronically adiabatic limit). In this case, the reaction occurs on the ground state free energy surface, which can be described as a function of $r, X$, and $R$ (Fig. 16). ${ }^{255}$ Quantization of the transferring proton coordinate results in a vibronic free energy surface that depends on only $X$ and $R$, as well as the applied electrode potential $E$. For each value of $E$, the free energy barrier corresponding to the saddle point relative to the reactant minimum on this two-dimensional vibronic free energy surface can be computed. ${ }^{255}$ Within a transition state theory framework, the rate constant depends exponentially on this free energy barrier, and the transfer coefficients can be computed.

This vibronically adiabatic approach was applied to the Volmer reaction in acidic aqueous solution. ${ }^{255}$ In this application, an empirical valence bond model was developed to generate the ground electronic state free energy surfaces at each applied potential $E$, followed by quantization of the proton and calculation of the free energy barrier on the vibronic ground state free energy surface. Most of the parameters in this model can be computed using first-principles simulation methods, although in some cases experimental data may be used as a guide. Typically the qualitative trends are not sensitive to the specific values of the parameters within physically reasonable ranges.
Within this model, the calculated transfer coefficients were consistent with experimental data, but the H/D KIEs were lower than the experimental values. Analysis of the vibronic free energy surfaces indicated that the splitting between the ground and first excited proton vibrational states on the electronic ground state was small enough at large $R$ to indicate a significant degree of vibrational nonadiabaticity. ${ }^{255}$ Neglect of the vibrational nonadiabaticity was proposed to be a plausible explanation for the underestimation of the H/D KIEs.

Thus, the vibronically adiabatic framework was modified to include the effects of the first excited adiabatic proton vibrational state. ${ }^{94}$ A unified formulation was developed to describe the Volmer reaction in terms of a curve crossing between the two diabatic vibronic states associated with the two lowest adiabatic proton vibrational states. ${ }^{94}$ In this formulation, the rate constant is expressed in the form

$$
k(R, E)=\nu(R) \exp \left[-\frac{\Delta G_{\mathrm{eff}}^{\ddagger}(R, E)}{k_{\mathrm{B}} T}\right]
$$

where $\nu(R)$ is a pre-exponential term and $\Delta G_{\text {eff }}^{\ddagger}$ is the effective activation free energy that includes the free energy required to reach the crossing point between the two diabatic curves as well as the work term required to bring the acid from bulk solution to a distance $R$ from the electrode surface. An interpolation scheme for $\nu(R)$ was devised to span the adiabatic transition state theory, nonadiabatic Fermi golden rule, and 


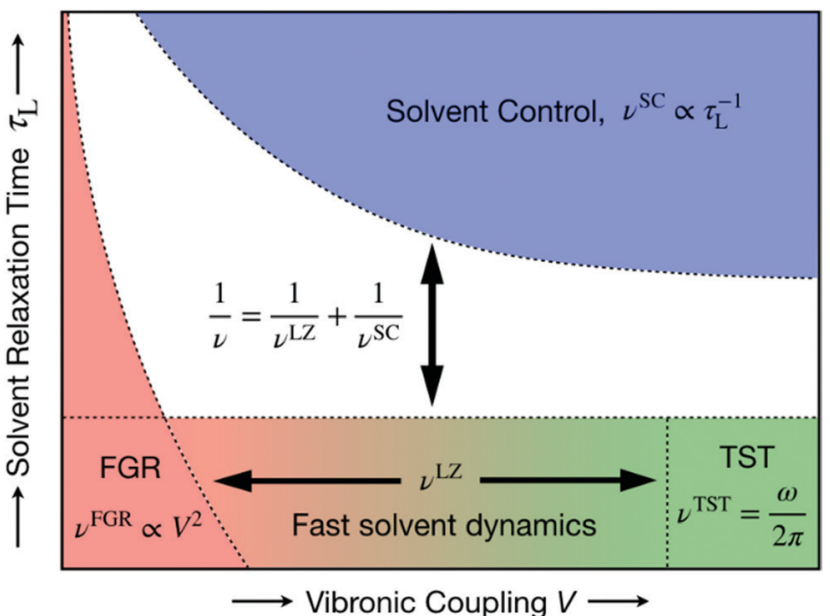

Fig. 17 Schematic representation of the unified formulation that accounts for both nonadiabaticity and solvent dynamics. In the limit of fast solvent dynamics (bottom of figure), the pre-exponent is given by $\nu^{L Z}$, which in the normal region interpolates between the nonadiabatic Fermi golden rule (FGR) limit $\nu^{\mathrm{FGR}}$ (bottom left) and the adiabatic transition state theory (TST) limit $\nu^{\text {TST }}$ (bottom right). In the limit of slow solvent dynamics (top), the pre-exponent is given by $\nu^{\mathrm{SC}}$. The model is valid for thermally activated processes in the two-state curve-crossing formulation. Reproduced with permission from ref. 94. Copyright 2019 American Chemical Society.

solvent-controlled regimes. ${ }^{94}$ This interpolation scheme is summarized in Fig. 17, and the specific mathematical expressions as well as a discussion of related interpolation schemes are given elsewhere. ${ }^{94}$ This theoretical formulation is closely related to the vibronically nonadiabatic formulation when $\nu(R)$ in eqn (9) is in the nonadiabatic Fermi golden rule limit (i.e., small vibronic coupling and short solvent relaxation time) and eqn (6) includes only the lowest reactant and product vibronic states with the vibronic coupling computed in the electronically adiabatic limit.

The interpolated curve-crossing approach was applied to the Volmer reaction in acidic aqueous solution using the empirical valence bond model described above. ${ }^{94}$ The lowest two adiabatic vibronic free energy surfaces along the collective solvent coordinate $X$ were diabatized for each distance $R$ between the acid and the electrode surface (Fig. 16), allowing the calculation of the interpolated prefactor $\nu(R)$, as illustrated in Fig. 10D. Subsequently, the rate constant $k(R, E)$ was calculated from eqn (9), and then the current density was calculated as a function of applied potential $E$ using eqn (5), as depicted in Fig. 10C. Analysis of the results indicated that vibrational nonadiabaticity and solvent dynamics play significant roles in this reaction. ${ }^{94}$ The calculated transfer coefficients of 0.69-0.73 and kinetic isotope effects of 3.8-7.1 are in reasonable agreement with experimental data ${ }^{46,261,262}$ for the Volmer reaction in acidic aqueous solution. Applications to the Volmer reaction in basic aqueous solution may require the development of additional methodology because of contributions from excited electronic states and thermodynamic effects from the formation of the hydroxide ion at the surface.

\section{Grand canonical ensemble DFT for electrochemical rate computation}

\subsection{Recent issues in DFT calculation for electrochemical reactions}

The electrode potential is at the heart of all electrochemistry as it offers a direct way to control electrochemical thermodynamics and kinetics. In principle, explicit control of the electrode potential is the only factor separating electrochemical and electrocatalytic reactions from all other "normal" chemical reactions. At the microscopic level, the electrode potential gives direct access to changing the chemical potential of electrons $(\mu)$ which in turn is directly proportional to the Fermi-level: $\mu \propto-E_{\mathrm{F}}{ }^{62}$ Given the importance of the electrode potential, it has been widely acknowledged that computational studies of electrochemical systems should be conducted at fixed electron chemical potential conditions. As discussed shortly below in Section 6.2, first principles calculations on electrochemical thermodynamics can be rigorously defined in the grand canonical ensemble (GCE), and a variety of approaches have been developed to realize or approximate the GCE.

While GCE has emerged as the gold standard in first principles computational studies on electrochemical thermodynamics, only a handful of works have focused on kinetics. The scarcity of studies on electrochemical kinetics can probably be attributed to both methodological and theoretical complexities. ${ }^{263}$ In particular, a generally valid rate theory for electrochemical systems has only recently been developed ${ }^{66}$ showing how electrochemical rate constants can be computed within the GCE. The GCE rate theory (GCE-RT) formalism allows inclusion of nonadiabatic and nuclear tunneling contributions as a function of the electrode potential using general first principles Hamiltonians. It has also been shown that GCE-RT enables the extension of all theoretical methods developed for studying tunneling in "normal" chemical reactions to be utilized for electrochemical reactions.

The emphasis of this chapter is on showing how firstprinciples methods, especially GCE-DFT (see Section 6.2), can be utilized to address reaction rates and nuclear quantum effects (NQE) as a function of the electrode potential using GCE-RT. The discussion presented here serves both as a perspective as well as a review and tutorial of promising methods to address nuclear quantum effects at electrochemical interfaces using first principles Hamiltonians. The perspective part of this section is that electrochemical rates are obtained by extending the canonical rate theories to the GCE. This provides a theoretically rigorous way to compute rate constants from first principles as a function of the electrode potential. Starting from this general GCE rate theory it is shown how well-defined approximations to treating nuclear tunnelling as a function of the electrode potential can be developed. Instead of giving a historical account, this section aims to review and connect different theoretical approaches in a pedagogical manner. The treated methods have been utilized to study condensed phase chemistry but many of them have not yet been applied to electrochemical reactions and it is shown here how this extension is achieved within GCE-RT. More traditional model 
Hamiltonian-based approaches are discussed in other parts of the work (Sections 5 and 7). This section will serve a three-fold purpose: (1) to introduce general rate theory to researchers working on experimental or computational electrochemistry, (2) to show how nuclear quantum effects can be addressed with computational tools used in heterogeneous (electro)catalysis, and (3) to inspire joint experimental-computational studies of quantum phenomena at electrochemical interfaces.

\subsection{The grand canonical ensemble for electrochemistry}

Properties of electrochemical interfaces are controlled by the electrode potential and the solvent or equivalently the (electro)chemical potential of electrons and the electrolyte, respectively. ${ }^{62}$ In macroscopic systems both the electrode potential and the solvent chemical potentials are constant, which means that electrochemical interfaces work under fixed electrochemical potentials. However, most of computational chemistry, such as density functional theory (DFT) calculations, are performed in the canonical or the $N V T$ ensemble characterized by constant temperature $(T)$, particle number $(N)$, and volume $(V)$ which lead to the Helmholtz free energy. ${ }^{264}$ In this $N V T$ ensemble the chemical potentials fluctuate, which makes it unsuitable for constant potential calculations. To achieve constant potential calculations, a Legendre transformation from the canonical ensemble to the GCE is performed to change the thermodynamic variables from $N V T$ to $\mu V T$.

It has been shown ${ }^{62}$ that in the $\mu V T$ ensemble, the thermodynamic state at fixed chemical potential is obtained rigorously from the GCE partition function $\Xi$ at chemical potential $\mu$ and nuclear-electronic Hamiltonian $\hat{H}_{\text {tot }}$ as $\Xi=\operatorname{Tr}\left[\exp \left[-\hat{H}_{\text {tot }}+\mu \hat{N}\right]\right]$ (Tr denotes a quantum mechanical trace i.e. quantum mechanical analogue of phase-space averaging). As $\hat{H}_{\text {tot }}$ is the full Hamiltonian, all quantum and non-adiabatic effects of electrons and nuclei are included in this multicomponent definition. In the GCE all expectation values are given in terms of the GCE density operator $\hat{\rho}$ as $\langle O\rangle=\operatorname{Tr}[\hat{\rho} \hat{O}]$. In particular, the constant potential free energy $\Omega$ is a functional of the GCE density operator ${ }^{62}$ and the equilibrium free energy $\Omega[\mu, T, V]=\Omega[\hat{\rho}]$ of the nuclear-electronic quantum system is uniquely obtained by minimizing $\Omega[\hat{\rho}]$ over all densities at constant chemical potentials leading to GCE-DFT. In this $\mu T V$ ensemble the number of species is allowed to fluctuate and the corresponding relevant thermodynamic free energy is the grand or Landau free energy $\Omega$. Methods for approximating the exact electrochemical free energy from atomistic DFT simulations as a function of the electrode potential have been devised and include self-consistent field method, ${ }^{265}$ iterative approaches, ${ }^{64,266}$ and the potentiostat scheme. ${ }^{267}$ Many of these techniques have been reviewed in the recent reports, ${ }^{268}$ and in the present work formally exact approaches are treated in Section 7 while model Hamiltonian-based approaches are utilized in Section 5 .

\subsection{Direct computation of rate constants}

As discussed above, GCE provides a rigorous basis for first principles computation of electrochemical thermodynamics at fixed potentials. To extend the GCE treatment to kinetics as well, it has been recently shown that equilibrium rate constants at fixed potential kinetics can be exactly derived using a GCE extension to the canonical rate theory. ${ }^{66}$ It is well known that the exact thermal rate constant in the canonical ensemble is given by ref. 66, 264, 269 and 270

$$
k(N, V, T) Q_{0}=\frac{1}{2} \pi \int_{-\infty}^{\infty} \mathrm{d} E \exp [-\beta E] \sigma(E)
$$

where $\beta=1 / k_{\mathrm{B}} T, Q_{0}$ is the canonical partition function of the initial state, $E$ is energy, and $\sigma(E)$ is the microcanonical cumulative reaction probability at energy $E$. Exact thermal rate constants can also be written in terms of flux and side correlation functions. For instance, in terms of the flux-side $C_{\mathrm{fs}}(t)$, the side-side $C_{\mathrm{ss}}(t)$, or the flux-flux $C_{\mathrm{ff}}(t)$ correlation functions the rate constant can be written as ${ }^{264,269,270}$

$$
\begin{aligned}
k(N, V, T) Q_{0} & =\lim _{t \rightarrow \infty} C_{\mathrm{fs}}(t)=\lim _{t \rightarrow \infty} \frac{\mathrm{d}}{\mathrm{d} t} C_{\mathrm{ss}}(t) \\
& =\frac{1}{2} \int_{-\infty}^{\infty} \mathrm{d} t C_{\mathrm{ff}}(t)
\end{aligned}
$$

The different correlation functions are related to each other as $C_{\mathrm{ff}}(t)=\mathrm{d} / \mathrm{d} t C_{\mathrm{fs}}(t)=\mathrm{d}^{2} / \mathrm{d} t^{2} C_{\mathrm{ss}}(t)$. To obtain qualitative understanding on the correlation function let us focus on the fluxside function. The quantum mechanical expression is ${ }^{264,269,270}$

$$
\lim _{t \rightarrow \infty} C_{\mathrm{fs}}(t)=\operatorname{Tr}\left[\mathrm{e}^{-\beta \hat{H}} \hat{F} P\right]
$$

where $\hat{F}$ is the flux operator, and $P$ the projector operator. In analogy with the classical rate theory discussed below, eqn (12) can be understood as shown in Fig. 18 and as follows. One starts a quantum mechanical "trajectory" from a multidimensional dividing surface $\hat{h}\left[q^{\ddagger}\right]$, which separates the reactants and products, according to a quantum mechanical thermal distribution $\left(\mathrm{e}^{-\beta \hat{H}}\right)$. The flux through $\hat{h}\left[q^{\ddagger}\right]$ towards the product is measured by $\hat{F}$. The projector operator $P=\lim _{t \rightarrow \infty} \mathrm{e}^{i \hat{H} t / \hbar} \hat{h}\left[q^{\ddagger}\right] \mathrm{e}^{-i \hat{H} t / \hbar}$ indicates the Heisenberg time-evolution of the dividing surface measuring how many "trajectories" initialized at $\hat{h}\left[q^{\ddagger}\right]$ end up in the product region as time is advanced.

Eqn (10) and (11) form the formal starting point for developing well-defined approximations to the exact rate constant in both the canonical ensemble ${ }^{264,269,270}$ and the electrochemically relevant GCE. ${ }^{66}$ The explicit forms of the cumulative reaction probability and the correlation functions depend on the Hamiltonian (classical or quantum) and the intended application (adiabatic, non-adiabatic, deep or shallow tunneling, etc.). Below, the general equations are connected to various approximations and schemes for estimating nuclear quantum effects.

To transfer the machinery developed for canonical rate constants to the electrochemical GCE setting, the following 


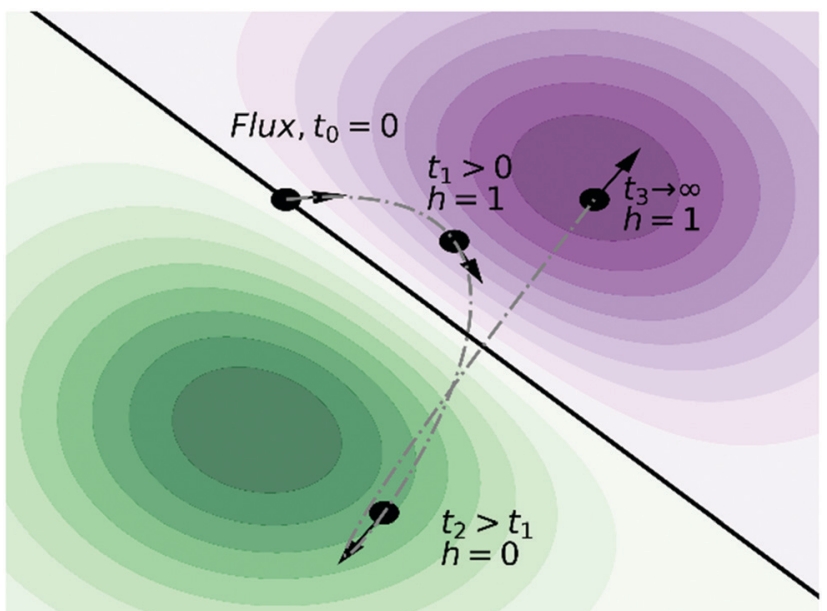

Fig. 18 A 2D free energy surface depicted with contours: the darker colors correspond to lower energies, purple is the product region, green is the reactant region and the thick black line is the dividing surface $h\left[q^{\ddagger}\right]$ between the reactants and products. A trajectory is initiated at the dividing surface at $t=0$ with a flux towards the products with momentum denoted by the arrow. The particle follows the trajectory as denoted with the grey dash-dotted line. At $t_{1}$ the particle is on the product side and the side function $h=1$. As time evolves the particle recrosses the dividing surface reactant side $\left(h=0\right.$ at $\left.t_{2}\right)$, and finally returns to the product side $(h=1)$ at $t_{3}$ making the trajectory a reactive one.

generalization is made ${ }^{66}$

$$
\begin{aligned}
k(\mu, V, T) \Xi_{0} & =\frac{1}{2} \pi \sum_{N=0}^{\infty} \exp [\beta \mu N] \int_{-\infty}^{\infty} \mathrm{d} E \exp \left[-\beta E_{N}\right] \sigma\left(E_{N}\right) \\
& =\sum_{N=0}^{\infty} \exp [\beta \mu N] k(T, V, N) Q_{0}=\lim _{t \rightarrow \infty} C_{\mathrm{fs}}^{\mu}(t)
\end{aligned}
$$

where $N$ is the particle number with corresponding energy $E_{N}$, $\Xi_{0}$ is the initial state GCE partition function, and $C_{\mathrm{fs}}^{\mu}$ denotes a GCE flux-side correlation function. These equations show that electrochemical kinetics can be understood and treated like "normal" chemical rates, and that electrochemical rate constants are just GCE averages of canonical rate constants. Furthermore, the last two equations show that all canonical rate theories and approaches can be generalized to the GCE and electrochemical reactions with general Hamiltonians using GCE averaging. ${ }^{66}$

\subsection{Adiabatic reactions}

In this subsection the focus is on reactions taking place on a single ground state potential energy surface (PES) and all excited states are neglected. To enable this, the Born-Oppenheimer approximation (see Section 7) is assumed and applied to separate nuclear and electron movement - the electrons are assumed to respond instantaneously to the nuclear displacement. In the treatment of proton-coupled electron transfer reactions where the protons are quantized, sometimes a double-adiabatic approximation is invoked (see Section 7) - the electrons and protons are considered to respond instantaneously to the movement of heavier nuclei. As discussed in Section 5, often the Born-Oppenheimer approximation is not valid for proton-coupled electron transfer reactions, and a vibronically nonadiabatic treatment is required. However, the BornOppenheimer approximation is the underlying assumption for all the rate theories covered in this section.

6.4.1 Classical rate constants and transition state theory. In the classical treatment the quantum character of all nuclei is ignored. The energies are obtained from a classical Hamiltonian as a sum of nuclear kinetic energy $K$ or the momenta $p$ and the potential energy $U$. In the canonical ensemble the classical Hamiltonian is $H_{\mathrm{cl}}\left(\boldsymbol{R}, N_{\mathrm{nuc}}, N_{\mathrm{el}}\right)=K_{\mathrm{nuc}}\left(N_{\mathrm{nuc}}\right)+$ $U\left(\boldsymbol{R}, N_{\text {nuc }}, N\right)=\sum_{i} p_{i}^{2} / 2 m+U\left(\boldsymbol{R}, N_{\text {nuc }}, N\right)$ and the dependence on the nuclear positions $\boldsymbol{R}$ and of the number of nuclei $\left(N_{\mathrm{nuc}}\right)$ and electrons $\left(N_{\mathrm{el}}\right)$ has been explicitly noted. In density functional theory or other first-principles calculations $U\left(\boldsymbol{R}, N_{\mathrm{nuc}}, N_{\mathrm{el}}\right)$ is solved directly as a function of nuclear positions and number of electrons, see Section 7. After introducing the classical Hamiltonian, flux and projections in eqn (12) gives the classical rate constant as $^{264}$

$$
k_{\mathrm{cl}}(T, V, N) Q_{0}=\frac{1}{(2 \pi \hbar)^{N}} \int \mathrm{d} t \mathrm{~d} p \mathrm{~d} \boldsymbol{R} e^{-\beta H_{\mathrm{cl}}} F(p(t), \mathrm{R}(t)) P\left(\boldsymbol{R}^{\ddagger}, t\right)
$$

which has the same interpretation as eqn (12). To make the fundamental assumption in transition state theory (TST) one takes the instantaneous $t \rightarrow 0_{+}$limit of the projection $P$; this means that all trajectories towards the product from dividing surface $\boldsymbol{R}^{\ddagger}$ are reactive and there are no recrossings of the dividing surface. This results in the canonical rate constant in terms of the transition state partition function $Q^{\ddagger}$ or the Helmholtz free energy barrier $\Delta A .^{270}$

$$
\frac{k_{\mathrm{B}} T}{h} \frac{Q^{\ddagger}}{Q_{0}}=\frac{k_{\mathrm{B}} T}{h} \exp \left[-\beta \Delta A^{\ddagger}\right]
$$

The classical TST is the staple of all chemical kinetics. It characterizes the passage from an initial to final state separated by a (free) energy barrier along the minimum energy pathway (MEP) connecting the reactants and products. In computational heterogeneous catalysis and electrocatalysis, treated at constant particle rather than constant electrode potential, an additional approximation of including only the harmonic vibrational partition functions is typically used. This approximation leads to the harmonic $\mathrm{TST}^{271}$

$$
k_{\mathrm{cl}, \mathrm{hTST}}(T, V, N)=v_{n} \exp \left[-\beta\left(\Delta E^{\ddagger}+T \Delta S_{\mathrm{vib}}\right)\right]
$$

with the vibrational entropy $S_{\mathrm{vib}}$ computed from the vibrational frequencies, energy barrier $\Delta E^{\ddagger}$, and vibrational frequency $v_{n}$ along the reaction coordinate.

Extension of the canonical classical rate theory to electrochemical and electrocatalytic situation is rather straight-forward. ${ }^{66}$ The formalism remains exactly the same apart from a modified PES and classical Hamiltonian; the potential energy becomes dependent on the fluctuation number of electrons at a fixed chemical potential, $U\left(\boldsymbol{R}, N_{\text {nuc }}, \mu\right)$. While this extension seems simple, 
in fact an additional Born-Oppenheimer-like approximation is needed as the change in number of electrons is assumed to be instantaneous and the electron transfer rates within the electrode or from the bulk to surface the are assumed to be infinitely fast. As such, the extension to GCE may break down for e.g. poorly conduction semiconductor electrodes. If the electron transfer within the electrode is fast, the fixed potential TST is simply in terms of GCE partition functions or the change in the grand canonical energy $\Delta \Omega^{66}$

$$
k_{\mathrm{cl}, \mathrm{TST}}(T, V, \mu)=\frac{k_{\mathrm{B}} T}{h} \frac{\Xi^{\ddagger}}{\Xi_{0}}=\frac{k_{\mathrm{B}} T}{h} \exp \left[-\beta \Delta \Omega^{\ddagger}\right]
$$

While the rigorous theoretical treatment of the GCE-TST has only recently been demonstrated, the formalism has already found use in fixed potential DFT studies of electrocatalytic reactions in static environments ${ }^{64-66,266,272-275}$ using a constant potential modification of the NEB method. ${ }^{276}$ Also, calculations with dynamical solvent have been performed ${ }^{277,278}$ using the blue moon ensemble ${ }^{279}$ method. Pioneering studies have shown the importance of analyzing the kinetics at constant potential rather than constant particle number as well as demonstrated the viability of GCE-TST in computing electrocatalytic rate constants from first principles for a wide class of electrocatalytic PCET reactions such as for hydrogen evolution, ${ }^{64,65,272,275} \mathrm{CO}_{2}$ reduction, ${ }^{266,280} \mathrm{CO}$ reduction, ${ }^{281}$ $\mathrm{NH}_{3}$ oxidation, ${ }^{282}$ nitrogen reduction, ${ }^{283}$ and oxygen reduction reactions, ${ }^{277}$ for example. Yet, all these first principles studies thus far have focused on classical reactions and neglected e.g. nuclear quantum effects which are known to be important in several PCET reactions.

6.4.2 Addressing nuclear quantum effects. A distinct advantage of the GCE-RT is its generality which highly beneficial for developing methods to treat nuclear tunneling under electrochemical conditions. While the full quantum mechanical rates can be understood and, in principle, evaluated using eqn (10) and (11), they can be solved for only simple model systems. For this reason, several approximate methods have been developed to address NQEs in realistic and complex condensed matter systems as discussed also in Sections 5 and 7. With regards to reaction rates, the most important quantum phenomena are zero-point energy (ZPE) and tunneling. ${ }^{284}$ While ZPEs can be accounted for rather easily (at least in the harmonic approximation), tunneling contributions are trickier and different approaches should be chosen depending on e.g. the type of reaction and temperature.

A coarse division in treating tunneling is based on the notion of deep and shallow tunneling. ${ }^{285,286}$ The former can be considered as genuine through the barrier tunneling from a single quantum level and the reaction pathway is different than from classical nuclei - referred to as corner-cutting. Shallow tunneling, on the other hand, can be viewed as a quantum mechanical correction due to tunneling near the classical pathway due to nuclear delocalization at the ensemble level. ${ }^{285}$ The division between deep and shallow tunneling can be based on the instanton cross-over temperature $T_{\mathrm{c}}=\hbar \omega_{n} / 2 \pi k_{\mathrm{b}}$ which depends on the imaginary saddle point angular frequency $\omega_{n} ;{ }^{287,288}$ at temperatures below $T_{\mathrm{c}}$ deep tunneling is operational while at higher temperatures the shallow tunneling description is appropriate. To get a feel for the range of vibrational frequencies to enter the deep tunneling regime at room temperature, the imaginary wave number at the barrier top, $\tilde{v}_{n}=\omega_{n} / 2 \pi c$, should be larger than $1400 \mathrm{~cm}^{-1}$. However, this division into tunneling regimes should be viewed with caution, and general approaches will cover all regimes - the division is used as a modelistic tool to aid making approximations to the true quantum solution.

Below, some of the currently most promising approaches for treating both shallow and deep tunneling in condensed phases and matter are discussed. The included methods have been demonstrated to perform well in characterizing NQEs in canonical condensed phase systems. Their expected performance, range of validity, and attainable insight is then placed in an electrochemical perspective. However, before diving into theoretical and computational approaches, some mathematical and physical background on necessary concepts is introduced.

6.4.2.1 Concepts of path-integrals and semi-classical approximations. Given the impossibility of solving the Schrödinger equation for complex condensed phase systems, several alternatives, both exact and approximate, have been developed. Among the most suitable exact methods are Feynman path integrals $^{289-292}$ (PI) which are also amenable to both numerical and semi-classical approximations. In particular, PIs enable an alternative expression for the time-evolution of quantum systems as discussed in detail in Section 7. Using a PI propagator for a particle moving from $x_{\mathrm{i}}$ to $x_{\mathrm{f}}$ during time $t$, the quantum mechanical solution (propagation) and PIs are related as

$$
K\left(x_{\mathrm{i}}, x_{\mathrm{f}} ; t\right)=\left\langle x_{\mathrm{f}}\left|\mathrm{e}^{-\hat{H} t / \hbar}\right| x_{\mathrm{i}}\right\rangle=\int_{x_{\mathrm{i}}(t=0)}^{x_{\mathrm{f}}(t=t)} \mathcal{D}(x) \mathrm{e}^{i A[x(t)] \hbar}
$$

where $\mathcal{D}(x)$ denotes all possible classical pathways $s$ between $x_{\mathrm{i}}$ and $x_{\mathrm{f}} . K$ is interpreted as the amplitude of the transition and is related to the probability as $P\left(x_{\mathrm{i}} \rightarrow x_{\mathrm{f}}\right)=|K|^{2}$. The weight or importance of each pathway depends on the classical action along pathway $s$ on $A[x(s)]$ of eqn (18) and is highly useful for obtaining physical insight into tunnelling processes. First, in classical mechanics, extremum of the action defines the classical pathway. This is equivalent to Newtonian, Lagrangian, or Hamiltonian mechanics. Second, quantum particles can take any pathway between the end points. Third, it can be shown that the most important tunneling paths are those whose action changes very little between two distinct pathways. Fourth, the most important (tunneling) pathways are obtained from extremization of the action. These observations lead to import conclusions: (1) pathways close to classical ones can be considered as quantum corrections to classical rates and (2) nonclassical pathways with extremized actions correspond to tunneling pathways through barriers. ${ }^{289}$

Besides dynamical properties, PIs can be utilized for computing quantum mechanical (grand) canonical partition functions. This is related to the mathematical similarity between the propagator $\exp [-i H t \hbar]$ and the Boltzmann operator $\exp [-\beta H]$ which can 
be interpreted in terms of imaginary time $t=-i \beta \hbar$. This property leads to the definition of Euclidian action $^{289}$

$$
S[x]=\int_{0}^{\beta \hbar} \mathrm{d} \tau\left[\frac{m}{2}\left(\frac{\mathrm{d} x}{\mathrm{~d} \tau}\right)^{2}-U(x(\tau))\right]
$$

which can be used to define the partition function as $Q(\beta)=$ $\int_{x_{\mathrm{i}}(\beta \hbar=0)}^{x_{\mathrm{i}}(\beta \hbar)} \mathcal{D}(x) \mathrm{e}^{-i S[x] / \hbar}$. The sampled paths are cyclic such that the initial and final points are identical. Euclidian path integrals lend well to numerical simulations and are among the most useful concepts for evaluating quantum effects in condensed phases, ${ }^{289,293-295}$ and for instance, a recent PI molecular dynamics (MD) study has shown that water dissociation on Pt is greatly enhanced by quantum effects. ${ }^{296}$

Euclidian action is also particularly suitable for analytic approximations at the semiclassical limit for capturing the most important tunnelling phenomena as discussed below. As a particular example, expanding the action of a one-dimensional system to second order and using stationary phase integration to the PI leads to the well-known WKB transmission probability ${ }^{292,297,298}$

$$
T(E)_{\mathrm{WKB}}=\frac{1}{1+\mathrm{e}^{2 \theta}}
$$

where the barrier penetration integral is $\theta=\frac{1}{\hbar} \int_{U\left(x_{\mathrm{i}}\right)=E}^{U\left(x_{\mathrm{f}}\right)=E}$ $\sqrt{2 m(U(x)-E)}$ at constant energy.

6.4.2.2 Shallow tunneling. The shallow tunneling describes nuclear quantum effects around the classical pathway. Here we take the well-defined semi-classical transition state theory $^{264,269,270,297,299,300}$ (SC-TST) as the starting point to arrive at various approximations for treating quantum mechanical effects near the classical pathway. While SC-TST can in some cases be extended to treat deep tunneling, ${ }^{301,302}$ only shallow tunneling contributions are achievable from fully numerical first-principles approaches using perturbative approaches.

The formulation of SC-TST starts from eqn (10) and approximates the cumulative reaction probability with the semiclassical WKB-like transmission probability of eqn (20). Unlike the one-dimensional WKB-approximation, the general SC-TST works for multidimensional systems in which different coordinates are non-separable and the barrier penetration integral in SC-TST should be viewed as generalization of the WKB integral. For electrochemical reactions the equivalent of the SC-TST rate constant is obtained from GCE-RT and takes the form

$$
\begin{aligned}
k_{\mathrm{SC}-\mathrm{TST}}(T, V, \mu) \Xi_{0} & =\frac{1}{2 \pi \hbar} \sum_{\mathrm{N}} e^{\beta \mu \mathrm{N}} \sum_{n^{\ddagger}} \int_{-\infty}^{\infty} \mathrm{d} E \frac{\mathrm{e}^{-\beta E}}{1+\mathrm{e}^{2 \theta\left(E, n^{\ddagger}\right)}} \\
& =\frac{k_{\mathrm{b}} T}{h} \Xi_{\mathrm{SC}-\mathrm{TST}}^{\ddagger}
\end{aligned}
$$

where $n^{\ddagger}$ are the vibrational quantum numbers at the transition state and $\Xi_{\text {SC-TST }}^{\ddagger}$ is a modified transition state partition function. As such, the SC-TST methods require first the identification of the classical transition energy barrier and then making corrections to it. In molecular applications, the transition state energy can be expanded using vibrational perturbation theory which leads to quantum mechanical corrections due to quantized vibrations near the classical barrier top. Along this route, a harmonic approximation to the energy was developed ${ }^{303}$ to give an expression suitable and manageable for condensed phases. For GCE, the SC-hTST rate constant is

$$
k_{\mathrm{SC}-\mathrm{hTST}}(T, V, \mu)=\frac{k_{\mathrm{b}} T}{h} \frac{\Xi^{\ddagger}}{\Xi_{0}} \gamma\left(T, v_{n}^{\ddagger}, \Delta E^{0}\right)
$$

which has the same structures as the classical hTST in eqn (17) multiplied by a tunneling correction $\gamma$, which depends on the barrier frequency and the ZPE corrected energy barrier $\Delta E^{0}$. The partition functions are for the quantum mechanical harmonic oscillator and therefore include ZPE; inclusion of the ZPE in the vibrational partition function can be considered as the simplest quantum correction to the classical rate constant. Unfortunately, in some literature the inclusion of ZPE is denoted as semi-classical TST which is inconsistent with the preceding discussion of the true SC-TST. As the vibrational partition function and the tunneling contribution are easy to evaluate, SC-hTST is readily applicable to analyze shallow tunneling in electrochemical interfaces as shown in Section 6.5. The promising SC-hTST is easily combined with DFT calculations to give at least qualitatively accurate understanding of shallow tunneling in zeolites ${ }^{303}$ and metals ${ }^{304,305}$

An even simpler analytic form that can be derived from SC-TST is the Wigner correction, which in quantum chemistry is taken as the first approximation of shallow tunnelling effects. The Wigner correction is derived for a one-dimensional parabolic barrier at temperatures well above $T_{\mathrm{c}}$ and given by $\gamma_{\mathrm{W}}=h \beta v_{n}^{\ddagger} / 2 \sin \left(h \beta v_{n}^{\ddagger} / 2\right)$. The Wigner correction diverges as $T_{\mathrm{c}}$ is approached from above, and close to $T_{\mathrm{c}} \mathrm{SC}-(\mathrm{h}) \mathrm{TST}$ provides a more robust alternative.

6.4.2.3 Deep tunneling. In the deep tunneling regime, the reaction pathway differs from the classical path and takes place through the barrier. This section focuses on two widely used PI-based methods which can describe electrochemical deep tunneling when combined with GCE rate theory: a semi-classical approximation to the propagator $K$ resulting in the instanton rate theory and ring-polymer molecular dynamics (RP-MD) which enables numerical evaluation of correlation functions from PI-based molecular dynamics. It is noteworthy that both approaches lead to similar rate expressions in the deep-tunnelling region and are equivalent to genuine quantum mechanical TST. ${ }^{306-308}$

The instanton rate theory ${ }^{297}$ can be derived using a semiclassical approximation to the PI propagator. ${ }^{298,309,310}$ Starting from eqn (11) the time-evolution is replaced by the imaginary time as discussed in Section 6.2.1. The initial and final positions are equal at the dividing surface, but the pathway connecting them is chosen to go through the classical barrier which is described as through barrier tunneling. The imaginary time trajectories are approximated by a semi-classical stationary phase approximation to the PI propagator which is exact for parabolic functions. This results in the instanton rate constant

$$
k(T, V, N)_{\text {instanton }} Q_{0}=A \mathrm{e}^{-S[x] / \hbar},
$$


where $A$ is a stability factor related to the vibrations along the harmonic directions. At low temperatures below $T_{\mathrm{c}}$ the rate is dominated by regions of small $S$ and the most probable tunneling pathway is obtained by minimizing the action. This defines a minimum action pathway (MAP) which is analogous with classical TST and the MEP. As stationary phase integration was employed, the instanton rate constant is exact for parabolic functions and therefore eqn (23) can be considered as the harmonic approximation to the MAP or as a harmonic quantum TST. The instanton rate theory is determined by the largest action along MAP similar to the (free) energy barrier in TST. Typically, the pathways that minimize the action are delocalized and located away from the classical pathway; this can be taken to represent delocalization of the nuclei through the barrier i.e., tunneling. Last, in one dimensional systems, the tunneling probability obtained from the instanton approach is equal to the WKB description. ${ }^{309,311}$

In recent years, instanton calculations combined with first principles electronic structure methods have started to become more frequent. This adaptation has been enabled by more robust numerical implementations describing the action and optimizing MAPs. The numerical presentation requires a discretization of the action, and currently the most robust discretization is based on a ring polymer representation of the cyclic instanton path $^{308,312}$ (see below). Based on the RP discretization effective algorithms for locating MAPs have been developed. ${ }^{313-316}$

The instanton rate constant is appropriate for deep tunneling only well below $T_{\mathrm{c}}$. Another limitation is the harmonic MAP approximation, which precludes the use of the instanton theory on highly anharmonic systems or liquids. Note that a similar restriction applies also to hTST which is the de facto standard for heterogeneous (electro)catalytic systems. Therefore, the instanton theory is expected to work well for describing deep tunneling in surface catalyzed reactions. Indeed, one of the first applications of the instanton rate theory with DFT calculations was $\mathrm{H}_{2}$ dissociation on $\mathrm{Cu}$ at low temperatures. ${ }^{317}$ Other solid-state examples of the method include kinetics of e.g. hydrogen diffusion in metals $^{318}$ and double-hydrogen transfer on metal supported porphycene. ${ }^{319}$ Extending the canonical instanton theory to electrochemical systems is straightforward - the only needed modification is to use the effective constant electrode potential PES given by $U\left(R, N_{\text {nuc }}, \mu\right)$ as in the classical TST case.

To address deep tunneling in liquid environments and to account for anharmonicities, the instanton approach can be replaced with another PI method, namely the ring-polymer (RP) approach. RP methods rely on the isomorphism between ringlike polymers and PIs and enable one to use an extended phase where each point-like classical nucleus is allowed to delocalize to represent the uncertainty principle. This delocalization is achieved by splitting a nucleus into several pieces (beads) connected to each other with harmonic springs so that the beads form a closed loop. With this presentation, the classical Hamiltonian is transformed to the RP Hamiltonian of the form

$$
H_{n}(\boldsymbol{p}, \boldsymbol{R})=\sum_{i=1}^{n}\left[\frac{p_{i}^{2}}{2 m}+\frac{1}{2} m \omega_{n}^{2}\left(\boldsymbol{R}_{j}-\boldsymbol{R}_{j+1}\right)^{2}+U(\boldsymbol{R})\right]
$$

with $\omega=\beta / n$ and $n$ is the number of beads. The use of RPs for including NQEs in partition functions computed from molecular dynamics (MD) was demonstrated already almost $40^{320}$ years ago. The breakthrough in using RPMD for computing reaction rates was the realization that the real time correlation functions can also be approximated from RPMD; ${ }^{321-323}$ this made it possible to compute the flux functions of eqn (11) in the extended RP phase space using RPMD and to include the NQEs in chemical rate constants. The RPMD approach has several distinct advantages as it works both in the shallow and deep tunneling limits and reduces to classical MD at high temperatures. RPs also capture both the ZPE and tunneling, the two most important quantum phenomena in rates. Also, the RPMD computed flux functions are well-defined at the short $t \rightarrow 0_{+}$limit which means that RPMD can be used to formulate a RP-TST which corresponds exactly to the quantum transition state theory. ${ }^{306}$ If a harmonic expansion of the RP-TST is performed, the instanton rate theory is obtained. ${ }^{308}$ However, typically the RPMD approach assumes the BornOppenheimer separation between the electrons and the nuclei. Therefore, the RPMD approach assumes that the nuclei move on the electronic ground state and is unable to describe electronically nonadiabatic processes without modifications that are not rigorous.

The RPMD method has proven to be a powerful method for studying quantum effects of liquids. ${ }^{294,295}$ Also, several proton and proton-coupled electron transfer reaction have been addressed in the liquid phase by using RPMD. ${ }^{324-326}$ In the solid state, hydrogen diffusion in ${ }^{318}$ and on $^{327}$ metals have been studied. Given the applicability of RPMD for modelling metals, surfaces, and liquids it is presumable that RPMD will also emerge as a powerful technique for studying electrochemical and electrocatalytic reactions. Again, the generalization to electrochemical reactions is accomplished by using the effective GCE PES given by $U\left(\boldsymbol{R}, N_{\text {nuc }}, \mu\right)$ or from computing the flux-side of eqn (13) with the extended RP Hamiltonian. While RPMD, WKB, and instanton rate theories are yet to be applied to electrochemical reactions, other PI-based methods have recently provided insight into the importance of nuclear quantum effects at electrochemical interfaces. $^{296}$

\subsection{HER from first principles}

After reviewing the GCE-DFT and GCE-RT approaches to adiabatic classical and quantum mechanical rate constants, utility of this machinery is demonstrated for the hydrogen evolution reaction (HER) - arguably the simplest and most important electrocatalytic PCET reaction. Yet, its atomic-level mechanism and kinetics still remain elusive despite numerous experimental, computational, and theoretical studies. As discussed in Section 6.2, constant electrode potential first-principles simulations enable the study of electrochemical reactions at complicated electrified solid-liquid interfaces without empirical parameters. Thus far, simulations have focused largely on electrocatalytic thermodynamics, but lately also studies on kinetics have started to appear. 
A highlight on what can be achieved using the GCE-RT and GCE-DFT methods in electrocatalysis has been recently provided by the Peterson group. ${ }^{64,65}$ In their work, the GCE-DFT method implemented using the surface-jellium model and the $\mathrm{NEB}^{276}$ method were utilized to understand the acidic HER on smooth gold and platinum surfaces with classical GCE-hTST rates. Besides demonstrating that it is technically possible to address complex and complete electrocatalytic pathways at electrochemical interfaces as a function of the electrode potential, these studies have shown that even the prototypical Pt-catalyzed HER cannot be fully understood from a thermodynamic perspective. Instead, the excellent performance of Pt as compared to e.g. Au can result from potential-dependent kinetics. Inclusion of kinetics has also enabled new mechanistic insight into the role of top and hollow bound hydrogens on both $\mathrm{Au}$ and Pt. Using a novel GC-formulation of the Marcus-like empirical valence bond (EVB) model, Melander has shown ${ }^{66}$ that potential-dependent reaction barriers obey a Marcus-like dependency on the reaction grand canonical energy. Besides the barrier, the GCE-EVB model was also successfully parametrized using constrained $\mathrm{DFT}^{328,329}$ to compute the solvent reorganization energy at the water-gold interface and estimate the TST geometry as a function of the electrode potential using just a single NEB calculation as a reference.

As a PCET reaction HER is a potential candidate for observing NQEs. We address treating hydrogen tunneling in Pt-catalyzed HER in acidic solutions using the methodology adopted from ref. 64 and 65. For the first i.e. Volmer step, the proton vibrational frequencies at the transition state geometry are very low, around $50-450 \mathrm{~cm}^{-1}$ depending on the potential. ${ }^{64,65,330}$ Based on the vibrational frequencies, the crossover temperatures are around $40 \mathrm{~K}$ and therefore deep tunneling is not expected at room temperature. At room temperature shallow tunneling contributions based on either the SC-hTST or Wigner model are also negligibly small. Also, the KIE computed from the Wigner correction and harmonic TST is small, below 1.1 for all electrode potentials. The second HER step is the Heyrovsky reaction, which is predicted ${ }^{65,331}$ as the rate limiting step for Pt-catalyzed HER. The Heyrovsky reaction exhibits somewhat larger imaginary vibrational frequencies which are in the range of $400-800 \mathrm{~cm}^{-1}$ depending on the potential. The reaction is still not in the deep tunnelling regime as $T_{\mathrm{c}}$ is below $200 \mathrm{~K}$. Using the Wigner expression, the KIE for the Pt-Heyrovsky reaction is around $1.4-1.5$ in nice agreement with the experiments $^{65,331}$ with Heyrovsky as the rate limiting step. The nuclear quantum effects in acidic Pt-catalyzed HER seem negligible and can likely be understood from the adiabatic shallow tunneling perspective.

While the above discussion provides evidence that HER on Pt can be understood from classical TST and that NQEs seem negligible for reaction kinetics, results from PIMD simulations show that NQEs might be important for water structures and interfacial pH. ${ }^{296}$ In particular, water dissociation is greatly facilitated on Pt but not on Au surfaces. As a result, $\mathrm{H}_{3} \mathrm{O}^{+}$and $\mathrm{OH}^{-}$ions concentrate on the Pt surface. Interestingly enough, deuterated water does not exhibit markedly-increased ionization upon inclusion of NQE. Furthermore, kinetic isotope effects for water dissociation were obtained from PIMD evaluated partition functions to parametrized PI-based quantum TST $^{332}$ (also known as centroid TST $^{333}$ ). This study demonstrated the applicability of PI techniques on realistic electrochemical solid-liquid interfaces as well as the complex and unexpected nuclear quantum phenomena on surface- and isotope-dependent solvent effects at room temperature.

Application of electrocatalytic rate theory has enabled a firstprinciples computational approach to study reaction trends, mechanisms, and kinetics as a function of the electrode potential. In the Pt-catalyzed HER, electronic quantum effects are crucial while the nuclear degrees of freedom can be treated classically, and small NQEs can be understood using the adiabatic shallow tunneling perspective. Yet, the dynamic water structure and dissociation exhibit NQEs, and their importance depends on the surface. These observations highlight the complexity of solid-liquid interfaces and surprising sources of quantum effects.

\subsection{Summary and remarks for this section}

In this section we have shown how well-defined electrochemical rate constants at a fixed electrode potential are achieved by extending canonical rate theories to the GCE. This facilitates following well-defined route to classical TST as well as to treating nuclear quantum effects from a set of fundamental equations and concepts. The GCE rate theory and approximations to it can be combined with general first principles Hamiltonians and DFT methods as the formalism is not tied to any model Hamiltonians. Different approximations to the GCE-RT to treat classical nuclei and nuclear tunneling within shallow and deep tunneling conditions were discussed and the validity of different approximations are recapped in Fig. 19. The discussion has focused on electronically adiabatic reactions, but non-adiabatic reactions can also be treated within the general GCE rate theory. ${ }^{66}$ Novel rate theories for including non-adiabatic effects in instanton theory $^{334,335}$ and RPMD ${ }^{284,293,336}$ exist but thus far they have been applied only to model systems. Therefore, the non-adiabatic reactions are presented within the more established curvecrossing picture in Sections 5 and 7.

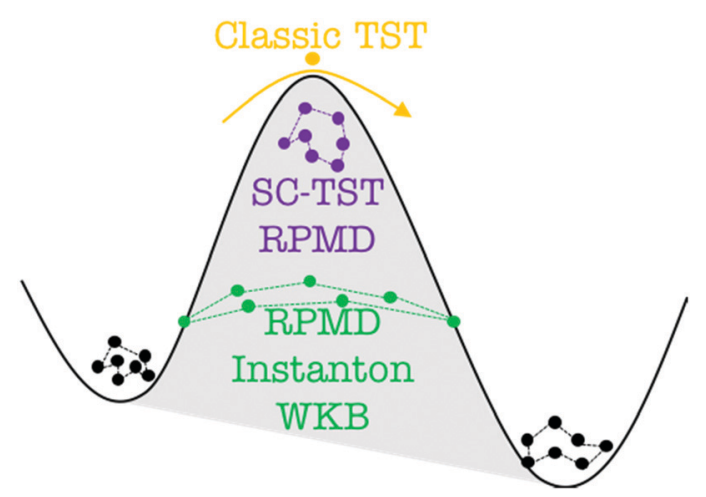

Fig. 19 Overview of the adiabatic tunnelling methods. The ring polymer size is proportional to the anticipated quantum tunnelling effects and the grey area is the classically forbidden area under the barrier. 


\section{First-principle calculations of quantum effects in proton transfer electrode processes}

7.1. Quantum many-body theories in electrode process science

The fuel cell reaction, a process to generate current from hydrogen and dioxygen $\left(2 \mathrm{H}_{2}+\mathrm{O}_{2} \rightarrow 2 \mathrm{H}_{2} \mathrm{O}\right)$, is comprised of charge transfer processes that occur at the electrode. A typical example is the electroadsorption of a proton described by $\mathrm{H}_{3} \mathrm{O}^{+}+\mathrm{e}^{-} \rightarrow \mathrm{H}^{*}+\mathrm{H}_{2} \mathrm{O}$ called the Volmer step of the hydrogen evolution reaction (HER), which is a PCET reaction (see Fig. 4 in Section 3 for a schematic image and Section 5 for the basic concepts and classifications for PCET). ${ }^{337-339}$ HER is obviously the simplest and the most studied reaction, but the understanding remains incomplete. This reflects the fact that the reaction cannot be simply captured by the formation and breaking of the chemical bond but needs to be captured with the strongly coupled environment. When the adsorbed hydrogen atoms, $\mathrm{H}^{*}$ 's, subsequently associate on the surface as $2 \mathrm{H}^{*} \rightarrow \mathrm{H}_{2}$, it is called the Tafel step of HER (Fig. 20). When another electroadsorption occurs on the adsorbed $\mathrm{H}^{*}$ as $\mathrm{H}^{*}+\mathrm{H}_{3} \mathrm{O}^{+}+$ $\mathrm{e}^{-} \rightarrow \mathrm{H}_{2}+\mathrm{H}_{2} \mathrm{O}$, it is called the Heyrovsky step of HER. These steps compete delicately on different surfaces. When the reaction occurs reversely on the hydrogen fuel cells, it is called hydrogen oxidation reaction that occurs at the anode side. At the cathode side, oxygen molecules are successively reduced by charge transfer processes via the reaction intermediates, $\mathrm{O}_{2}{ }^{*}, \mathrm{OH}^{*}, \mathrm{O}_{2} \mathrm{H}^{*}$, and $\mathrm{O}^{*}$. Their balance needs to be understood carefully. Despite these difficulties, it has long been the dream of theoreticians to establish the theory based on the sophisticated quantum many-body theory. Toward this goal, theory has advanced step by step, and at this stage, it will be important to review how the theory has advanced and how it should be developed in the future.

\subsection{Theoretical picture of electrochemical reaction dynamics at solid/liquid interfaces}

7.2.1 Formal theory of nonadiabatic electrode dynamics. In the early stage, the perturbation theory was used for reactions occurring in the outer Helmholtz layer. Dogonadze et al. ${ }^{28}$

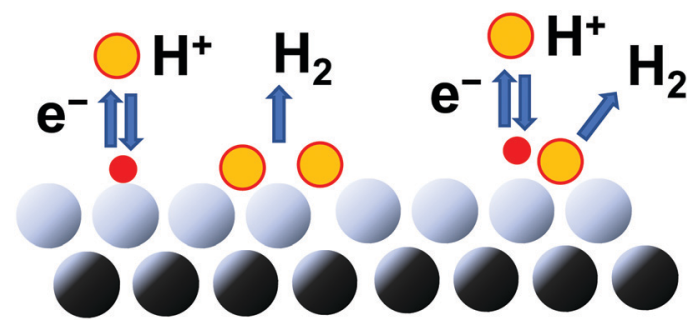

Fig. 20 Hydrogen evolution reaction on Pt(111). Hydrated proton is exchanged with electron in the electrode in the Volmer step (left). In the Tafel step, the adsorbed hydrogen atoms associate (middle), and in the Heyrovsky step, the Volmer step occurs on the adsorbed hydrogen atom (right). Filled red circles and yellow circles respectively indicate the adsorbed proton at the different position, i.e. ontop and hollow. The importance of adsorption sites are discussed later with Fig. 21. applied the Fermi golden rule

$$
R=\frac{2 \pi}{\hbar} \sum_{\mathrm{r}} \sum_{\mathrm{p}}\left|\left\langle\Psi_{\mathrm{p}}|V| \Psi_{\mathrm{r}}\right\rangle\right|^{2} \delta\left(E_{\mathrm{p}}-E_{\mathrm{r}}\right)
$$

to the reactant state $\Psi_{\mathrm{r}}$ weakly coupled to the product state $\Psi_{\mathrm{p}}$. This is a general quantum formula for the reactant $(\mathrm{r}) \rightarrow$ product (p) transition probability $(R)$. Here $V$ is the energy of the perturbation to cause the transition, and the $\delta$ function with an energy difference $E_{\mathrm{p}}-E_{\mathrm{r}}$ expresses the energy conservation law in the transition. Note that the wave functions, $\Psi_{\mathrm{r}}$ and $\Psi_{\mathrm{p}}$, contain full electronic and nuclear degrees of freedom and are extremely complex to handle. Practically, the wave function was simply decomposed into a product of the electronic $\Phi$ and nuclear $\chi$ parts, and the latter was subsequently approximated as a direct product of the wave function of the reacting proton and that of other nuclei. The resulting "double adiabatic" approximation neglects the coupling among electrons, reacting proton, and the environmental atoms and thus is insufficient for the electrochemical reactions. The coupling is overwhelmingly complex, as can be found in the study of PCET shown in Section 5.

Formal theory of the reaction dynamics can be provided from first principles within the path integral (PI) scheme. ${ }^{340}$ The reaction rate can be given as $\left\langle\boldsymbol{Q}_{\mathrm{p}}\left|\mathrm{e}^{-H \tau}\right| \boldsymbol{Q}_{\mathrm{r}}\right\rangle$, where $\boldsymbol{Q}_{\mathrm{r}}$ and $\boldsymbol{Q}_{\mathrm{p}}$ are the initial and final configurations, respectively, and $H$ is the full Hamiltonian $H(\boldsymbol{r}, \boldsymbol{R})$ for all the electronic $\boldsymbol{r}$ and nuclear $\boldsymbol{R}$ degrees of freedom. The rate is evaluated in PI by the set of nuclear paths $\boldsymbol{R}(t)$ for a time interval $\tau$, which is discretized into pieces $\Delta t$ as $\boldsymbol{R}(k \Delta t) \equiv \boldsymbol{R}_{k}$ with $0 \leq k \leq p$. The formulation is done by decomposing $H(\boldsymbol{r}, \boldsymbol{R})$ into the nuclear part

$$
H_{n}(\boldsymbol{R})=\frac{\boldsymbol{P}^{2}}{2 M}+U(\boldsymbol{R})
$$

and the electronic part

$$
H_{\mathrm{e}}(\boldsymbol{r}, \boldsymbol{R})=\frac{\boldsymbol{p}^{2}}{2 m}+V(\boldsymbol{r}, \boldsymbol{R}),
$$

and by extracting the operator to be applied to the reactant $\Phi_{\mathrm{r}}$ and product $\Phi_{\mathrm{p}}$ states of the electrons as

$$
\begin{aligned}
& \int \prod_{k=1}^{p} \mathrm{~d} \boldsymbol{R}_{k} \exp \left[-i \frac{\Delta t}{2}\left(H_{\mathrm{e}}\left(\boldsymbol{r}, \boldsymbol{R}_{k}\right)+U\left(\boldsymbol{R}_{k}\right)\right)\right] \\
& \times\left\langle\boldsymbol{R}_{k}\left|\exp \left[-i \Delta t \frac{\boldsymbol{P}^{2}}{2 M}\right]\right| \boldsymbol{R}_{k+1}\right\rangle \exp \left[-i \frac{\Delta t}{2}\left(H_{\mathrm{e}}\left(\boldsymbol{r}, \boldsymbol{R}_{k+1}\right)+U\left(\boldsymbol{R}_{k+1}\right)\right)\right] .
\end{aligned}
$$

This can be recast into the typical PI form, or the integration over all nuclear paths

$$
\int \mathcal{D} \boldsymbol{R}(\tau) \mathrm{e}^{-i S_{n}[\boldsymbol{R}(\tau)]} G[\boldsymbol{R}(\tau)],
$$

comprised of the nuclear action

$$
S_{n}[\boldsymbol{R}(t)]=\frac{1}{2} M \dot{\boldsymbol{R}}^{2}(t)-U(\boldsymbol{R}(t))
$$


and the potential part

$$
G[\boldsymbol{R}(\tau)]=\prod_{k=1}^{p} \exp \left[-i \Delta t H_{\mathrm{e}}\left(\boldsymbol{r}, \boldsymbol{R}_{k}\right)\right] .
$$

To evaluate $G[\boldsymbol{R}]$, a complete set is inserted between the exponentials. To allow nuclei to mix different Born-Oppenheimer (BO) states beyond the BO-PI scheme, ${ }^{341}$ it is convenient to use the Slater determinant consisting of nonorthogonal orbitals $\phi\left(\boldsymbol{r}, \boldsymbol{R}_{k}\right)$, that satisfy the coherent state condition ${ }^{342}$

$$
\left\langle\boldsymbol{r} \mid \phi\left(\boldsymbol{R}_{k}\right)\right\rangle=\exp \left[\phi\left(\boldsymbol{r}, \boldsymbol{R}_{k}\right) a^{+}\right]|0\rangle .
$$

With the complete set relation described as an integration over the orbitals

$$
\begin{aligned}
1 & =\int \frac{\mathrm{d} \phi^{*}(\boldsymbol{R}) \mathrm{d} \phi(\boldsymbol{R})}{2 \pi i} \exp [-\langle\phi(\boldsymbol{R}) \mid \phi(\boldsymbol{R})\rangle]|\phi(\boldsymbol{R})\rangle\langle\phi(\boldsymbol{R})| \\
& \equiv \int D\left[\phi^{*}(\boldsymbol{R}), \phi(\boldsymbol{R})\right]|\phi(\boldsymbol{R})\rangle\langle\phi(\boldsymbol{R})|,
\end{aligned}
$$

where ${ }^{*}$ means complex conjugate, one can rewrite the operator $G$ using two sets of orbitals, $\phi$ and $\psi$, provided respectively for bra and ket, as,

$$
\begin{aligned}
G[\boldsymbol{R}]= & {\left[\prod_{k} D\left[\phi^{*}\left(\boldsymbol{R}_{k}\right), \phi\left(\boldsymbol{R}_{k}\right)\right] D\left[\psi^{*}\left(\boldsymbol{R}_{k}\right), \psi\left(\boldsymbol{R}_{k}\right)\right]\right] } \\
& \left|\psi\left(\boldsymbol{R}_{1}\right)\right\rangle \exp \left[i S_{\mathrm{e}}\right]\left\langle\phi\left(\boldsymbol{R}_{\mathrm{P}}\right)\right|,
\end{aligned}
$$

using the electronic action

$$
S_{\mathrm{e}}=\sum_{k} \frac{\left\langle\phi\left(\boldsymbol{R}_{k}\right)\left|i \overleftrightarrow{\partial}_{\tau}-H_{\mathrm{e}}\left(\boldsymbol{R}_{k}\right)\right| \psi\left(\boldsymbol{R}_{k}\right)\right\rangle}{\left\langle\phi\left(\boldsymbol{R}_{k}\right) \mid \psi\left(\boldsymbol{R}_{k}\right)\right\rangle}
$$

with

$$
\begin{aligned}
& \left\langle\phi\left(\boldsymbol{R}_{k}\right)\left|\overleftrightarrow{\partial}_{\tau}\right| \psi\left(\boldsymbol{R}_{k}\right)\right\rangle \\
& =\frac{1}{2}\left[\left\langle\phi\left(\boldsymbol{R}_{k}\right) \mid \psi\left(\boldsymbol{R}_{k+1}\right)-\psi\left(\boldsymbol{R}_{k}\right)\right\rangle-\left\langle\phi\left(\boldsymbol{R}_{k}\right)-\phi\left(\boldsymbol{R}_{k-1}\right) \mid \psi\left(\boldsymbol{R}_{k}\right)\right\rangle\right] .
\end{aligned}
$$

Therefore, in the limit of an infinitesimal time interval, one can describe the action more symmetrically for nuclei and electrons

$$
S[\boldsymbol{R}(\tau)]=S_{n}[\boldsymbol{R}(\tau)]+\frac{\left\langle\phi(\boldsymbol{R}(\tau))\left|i \overleftrightarrow{\partial}_{\tau}-H_{\mathrm{e}}(\boldsymbol{R}(\tau))\right| \psi(\boldsymbol{R}(\tau))\right\rangle}{\langle\phi(\boldsymbol{R}(\tau)) \mid \psi(\boldsymbol{R}(\tau))\rangle}
$$

If the coherent states are approximately replaced by the Born-Oppenheimer (BO) state $\Phi$, the action becomes

$$
\frac{1}{2} M \dot{\boldsymbol{R}}^{2}(t)-E(\boldsymbol{R}(t))+\left\langle\Phi(\boldsymbol{R}(\tau))\left|i \overleftrightarrow{\partial}_{\tau}\right| \Phi(\boldsymbol{R}(\tau))\right\rangle,
$$

where $E(\boldsymbol{R})$ is the total energy within the BO approximation (BOA). The first two terms yield the time evolution within the BOA while the third term causes the non-adiabatic effect. When the density functional theory (DFT) is combined, the first two terms yield the $a b$ initio PIMD, and when the stationary phase approximation is applied further, the semiclassical $a b$ initio MD
(AIMD) results. One can incorporate some of the nonadiabatic effects into the classical dynamics thus formulated, thereby generating the equation of motion as

$$
M \ddot{\boldsymbol{R}}=-\nabla_{\boldsymbol{R}} E(\boldsymbol{R})+\text { (nonadiabatic terms). }
$$

There is a way to recover the quantum fluctuations neglected hereby, as was realized by the surface hopping method. ${ }^{343,344}$ Because of the large computational requirement, it is not yet practical to consider both the quantum and non-adiabatic effects unless using a simple model. Most of the recent fuel cell simulations, instead, have focused on the adiabatic time evolution occurring in the inner Helmholtz layer.

Before mentioning the adiabatic dynamics, the model-based approach for the rate of electron transfer reactions is worth discussion. In the Newns-Anderson-Schmickler model, ${ }^{345}$ the electrochemical interface is characterized by the density of states of the electrode and a level of the redox center. The model is found to reasonably well describe the electrochemical reactions including the Volmer step ${ }^{346}$ despite its simplicity. On this basis, Tanaka et $a l^{347}$ derived a field theoretical formulation to account for the reaction rate within the linear coupling regime of the redox level and the displacement of environmental coordinates. The derived formula, in the weak coupling limit, clearly demonstrates the importance of quantum mechanical effects. The model-based approach will play a role complementary to the fully ab initio methods in the future in view of the recent development of mapping density functional calculations to the Hubbard model systematically. ${ }^{348}$

7.2.2 Adiabatic electrode dynamics. The adiabatic approach based on the AIMD simulation was applied to the Volmer step by Otani et al. ${ }^{59}$ who set up a model ${ }^{57,267}$ where the Pt(111)-water interface is electrified negatively so that the reaction occurs exothermically. After equilibrating the interface following putting a hydronium ion into the liquid water, the water molecules were found to form a contact layer directing a hydrogen atom toward the surface. The hydronium ion then migrated towards the interface, and exchange was found to occur between a hydrogen atom of the hydronium ion and an electron of the Pt electrode. The reaction was followed by reorganization of the contacting water because the hydronium ion that had released the hydrogen atom now directs the oxygen atom toward the surface and prefers to rotate towards the initial configuration. To direct the hydrogen atom to the surface, the water molecule first left the contact layer to weaken the hydrogen bond network formed on the surface and subsequently returned after reorienting the molecular axis. The whole dynamics revealed by this simulation was later considered to be the rate determining step of the Volmer reaction. ${ }^{349}$ Adiabatic simulations reveal with increasing clarity the reaction paths and activation energy. ${ }^{350}$ It was reported that the Zundel form $\mathrm{H}_{5} \mathrm{O}_{2}{ }^{+}$is formed in contact with the surface and then leaves a hydrogen towards the solution with the largest barrier height of $0.26 \mathrm{eV}$. It is possible with the availability of supercomputers to perform the adiabatic simulation using many initial configurations.

7.2.3 Modeling of electric double layer. So far, firstprinciples study has rather focused on the adiabatic dynamics 
of the inner Helmholtz reaction as mentioned above, and the advance has been remarkably made in describing the electric double layer (EDL). The formation of the EDL is crucially important in determining the potential drop occurring between the electrode and the bulk solution. The EDL has a short-range component caused by surface sorption and solvation and a long-range component caused by redistribution of the electrolyte ions near the electrode-solution interface. The long-range component is the space charge layer issue that is beyond the scale reachable by first principles DFT simulation and hence has been preferentially described using a solvation model. Simple models such as the modified Poisson Boltzmann model were used in the early stage, ${ }^{351-353}$ but the yielded value for the interfacial capacitance was not so satisfactory. ${ }^{62}$ The most appropriate solution model is currently the one based on the reference site interaction model (RISM), ${ }^{354,355}$ where the density distribution of solute molecules, which are interacting with a force field model, is determined with the help of a functional relation called closure; RISM can be formulated rigorously with the exact closure, but approximate closures are used in practice. The RISM equation for the ionic density was combined with DFT for the electron density, so that both are determined self-consistently. ${ }^{356}$ The model can describe the potential profile of the electrode referring to the bulk solution and thus determine the bias potential microscopically. Indeed, Haruyama et al. ${ }^{61}$ applied the scheme to metal-solution interfaces to consistently relate the work required to transfer an electron from the electrode to the solution with the potential profile and its bias dependence. This enabled them to determine the reversible hydrogen electrode level theoretically. In view of the incapability of conventional force field models to accurately describe the solvation, ${ }^{357}$ however, the closure of RISM will need improvement. Note that, more recently, a similar solvation model was proposed using the classical DFT framework (see Section 6 for further reading). ${ }^{62}$

Electrochemical interfaces have been studied with increasingly elaborate description of the electric double layer, as reviewed comprehensively in recent literature. ${ }^{358-363}$ To avoid duplication, here we just summarize recent application of our method. The $a b$ initio molecular dynamics (AIMD) simulation was combined with ESM model to the problem of oxygen reduction reaction or water splitting on $\mathrm{Pt},{ }^{277,364-366}$ hydrogen evolution on $\mathrm{Ag}^{367}$ and an ionic liquid on $\mathrm{Li}^{368}{ }^{368}$ The constant chemical potential scheme was applied to the problem of redox level, ${ }^{369} \mathrm{Pt}$ electrode, ${ }^{110}$ and oxygen evolution reaction pathways. ${ }^{370}$ The ESM-RISM was applied to an oxidized $\mathrm{Pt},{ }^{371}$ and a graphitic surface. ${ }^{372}$ We point out that simple solvation models are preferred even today, ${ }^{373-375}$ while there is increasing number of researches that adopt the conventional periodicinterface-model where the associated boundary problem is reduced by incorporating more water molecules and solvated ions into the computational cell. ${ }^{64,376-378}$

\subsection{Quantum effect on hydrogen adsorption}

In addition to an accurate description of the EDL, the accuracy of sorption phenomena is very important. The potential energy

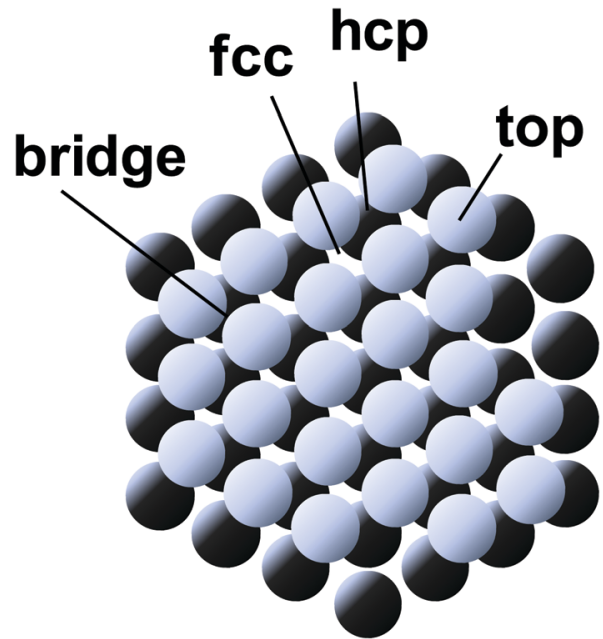

Fig. 21 Symmetric adsorption sites on Pt(111).

surface on the metal surface is generally flat on the order of 10-100 meV for different adsorption sites on Pt(111) (Fig. 21), which is comparable to the thermal energy at room temperature, $26 \mathrm{meV}$. The electronic structure calculation should be accurate to this energy scale, but it is not so easy to achieve this. It was shown that the adsorption of $\mathrm{CO}$ is inaccurate by $0.1 \mathrm{eV}$ on $\mathrm{Pt}(111)$ when using the generalized gradient approximation (GGA) or a semilocal approximation to the exchange-correlation energy. The problem apparent in the popular DFT simulations was overcome by using an advanced density functional called random phase approximation (RPA). ${ }^{379}$ When this calculation was applied to $\mathrm{H} / \mathrm{Pt}(111)$, hydrogen atoms more favorably occupy the fcc site at the full monolayer coverage condition, but the adsorption energy is comparable to the atop site within the energy scale of room temperature. This coexisting behavior of the adsorbed hydrogen atoms contrasts with the view provided by a GGA calculation that indicates the fcc site is considerably more stable. The qualitative difference in the adsorption feature should affect the kinetics of HER and HOR. This is because the hydrogen adsorption is considered to occur preferentially on the atop site so that the whole reaction is influenced by the subsequent hopping. If the fcc hydrogen predominantly exist as a stable species, it will exist as a spectator of the reaction. If the atop is predominant on the contrary, it will block the reaction as a poison. The coexistence means a possible flow of the atop to the fcc depending on the barrier height existing in between.

Quantum confinement is another important factor. This has been understood from the role of the zero-point energy (ZPE) in determining the most stable adsorption site. ${ }^{380,381}$ On the $\mathrm{Pt}(111)$ site under the monolayer coverage condition, hydrogen forms a monocoordinated $\mathrm{Pt}-\mathrm{H}$ bond on the atop site that is stronger than that formed on the three-fold coordinated fcc sites; however, the stability on the atop is less than that on the fcc because of the larger ZPE effect on the atop that overcompensates for the effect of classical binding energy.

The ZPE effect is included in the abovementioned calculations, but the confinement potential is quite anharmonic. ${ }^{382}$ The anharmonicity is considerably larger under sub-monolayer 


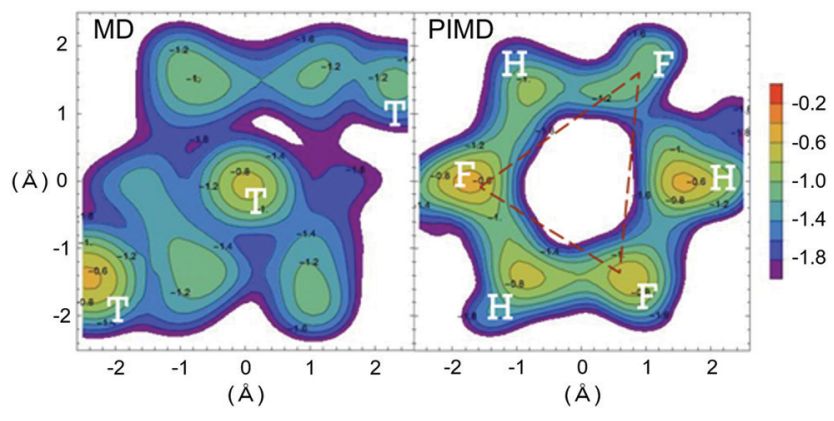

Fig. 22 Density distribution of $\mathrm{H}$ on $\mathrm{Pt}(111)$. The density has a maximum on the atop (T) site when simulated classically (a), while it has a maximum at the hollow sites ( $\mathrm{F}$ and $\mathrm{H})$ then simulated quantum mechanically (b). The figure was adopted with permission from the ref. 382. Copyright (2020) American Physical Society.

conditions than under the full monolayer condition because of the existence of a 'vacancy'. The vacancy also facilitates hopping of a hydrogen atom to a site nearby. According to the ab initio path integral molecular dynamics (PIMD) simulation done at room temperature (Fig. 22), ${ }^{382}$ the hopping occurs frequently under a $2 / 3$ monolayer, $\theta=2 / 3$, causing a delocalized feature in the density distribution. The density is maximum on the fcc and hcp hollow sites and is slightly smaller in the region in between, in sharp contrast to the density at the monolayer where the maximum in the density appears both at the atop and the fcc. This was interpreted using a lattice gas model augmented with the hopping between the nearest neighbor fcc and hep sites. The model is characterized by the strong $\mathrm{H}-\mathrm{H}$ repulsion and the nearly degenerate nature of the adsorption sites and thus has the property typical of strongly correlated many-body systems. The delocalization with reduced coverage and the resulting kinetic energy lowering are the typical property of the system; indeed, a similar property of hydrogen was found also on other metal surfaces (also see the references in ref. 324). ${ }^{383}$ In the PIMD simulation, the hydrogen put on the atop immediately hops to the hollow sites and is delocalized, ${ }^{384}$ so that the hollow sites should play a participating role in the Volmer step.

Although the research has been mostly limited to static properties like the adsorption site, the PI approach should be able to reveal dynamic properties as well. The role of quantum tunneling was studied mostly for surface diffusion, ${ }^{385}$ but such simulations can clarify the role of tunneling on the reaction.

\subsection{In silico electrocatalyst design}

To design the most efficient electrocatalysts, it is not practical to apply the sophisticated theory. For the purpose of screening, the most familiar method is the method based on the computational electrode model. ${ }^{386}$ Therein, the pristine surface is prepared to calculate the adsorption energy of the reaction intermediates: $\mathrm{H}^{*}$ for the anode and $\mathrm{O}^{*}, \mathrm{OH}^{*}, \mathrm{O}_{2} \mathrm{H}^{*}$ for the cathode. The energy of the hydrated proton is taken to be equal to the free energy of $\mathrm{H}_{2}$ in the gas phase at $1.23 \mathrm{Vvs}$. SHE and is increased by $x$ volt as the bias potential is reduced by $x$ volt. This allows one to approximately relate the adsorption energy with the reversible electrode potential. The theory explains simply the peak of the electrocatalytic activity versus the adsorption energy, known as the volcano relation, which is considered as a manifestation of the Sabatier principle; for higher activity the adsorption should not be too strong and not be too weak. The theory was used to explain why platinum is more active than other noble metals. ${ }^{386}$ The reason why it is difficult to overcome platinum was ascribed to the linear relation of the adsorption energy between $\mathrm{O}^{*}$ and $\mathrm{OH}^{*}$. The relation was shown to be applicable to many surfaces where the reaction intermediates take an adsorption structure common to the intermediates. There are, however, exceptions in some cases, including the doped $\mathrm{TiO}_{2}$ surface, ${ }^{387}$ which was expected to be a candidate for a superior electrocatalyst. The simple screening method thus has been successfully applied to many materials, but the success does not automatically justify the assumptions made in the theory; indeed, there are critical arguments. ${ }^{388}$ In this context a trial was made to replace the adsorption on a pristine surface with the average taken over possible configurations of the environment. ${ }^{389}$ It is now possible to do the thermodynamic sampling without mapping to a model owing to the development of ab initio Monte Carlo simulation. ${ }^{390}$ It is worth pointing out that such simulation should enable one to describe the space charge layer, or the EDL, formed on a semiconductor-based-electrode, although such electrode materials have only infrequently been studied theoretically. An important target of the theory will be therefore to make feasible the elaborate environmental description and then finally to enable simulation of the reaction dynamics.

\section{General conclusion and future outlook}

We have discussed a wide spectrum of recent advances and challenges in modern electrode process science at solid-liquid interfaces. As shown in the overall sections, the challenges in these systems are extraordinarily difficult and complicated, and on the other hand, the advancements still seem too little to understand the complicated microscopic mechanism of multielectron/-proton transfer electrode processes at solid-liquid interfaces. However, we have an advantage: the key research concepts and philosophy of fundamental electrode process science at solid-liquid interfaces are greatly affected by the surface science at solid-gas interfaces with model systems. ${ }^{50,391-396}$ It is well-known that these solid-gas systems brought greater understanding of a variety of basic catalytic mechanisms at single crystal and well-ordered thin film surfaces, as highlighted by the Nobel Prize for Gerhard Ertl in 2007. ${ }^{391}$ Therefore, we already have cumulative knowledge since the Ostwald era to import to solid-liquid systems. ${ }^{397}$ The well-established guidelines developed to tackle complicated solid-gas systems combined with experimental and theoretical approaches shown in the previous review ${ }^{49}$ allow us to investigate the microscopic principles of charge transfer reactions at solid-liquid interfaces using model systems combined with theoretical and spectroscopic approaches (Fig. 23). However, it is important to note that 


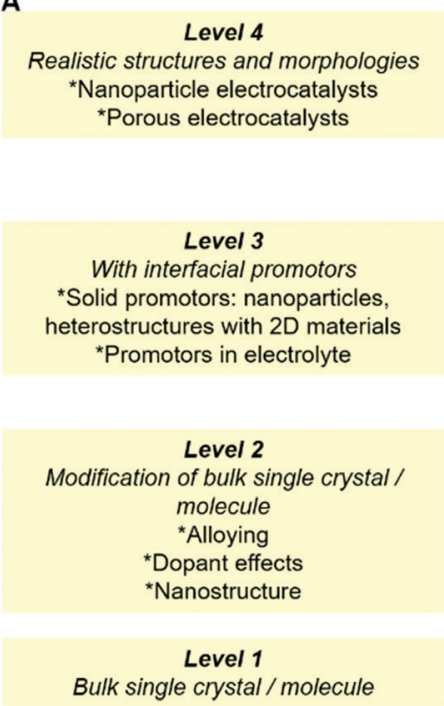

B

$$
\text { Yes }
$$

至

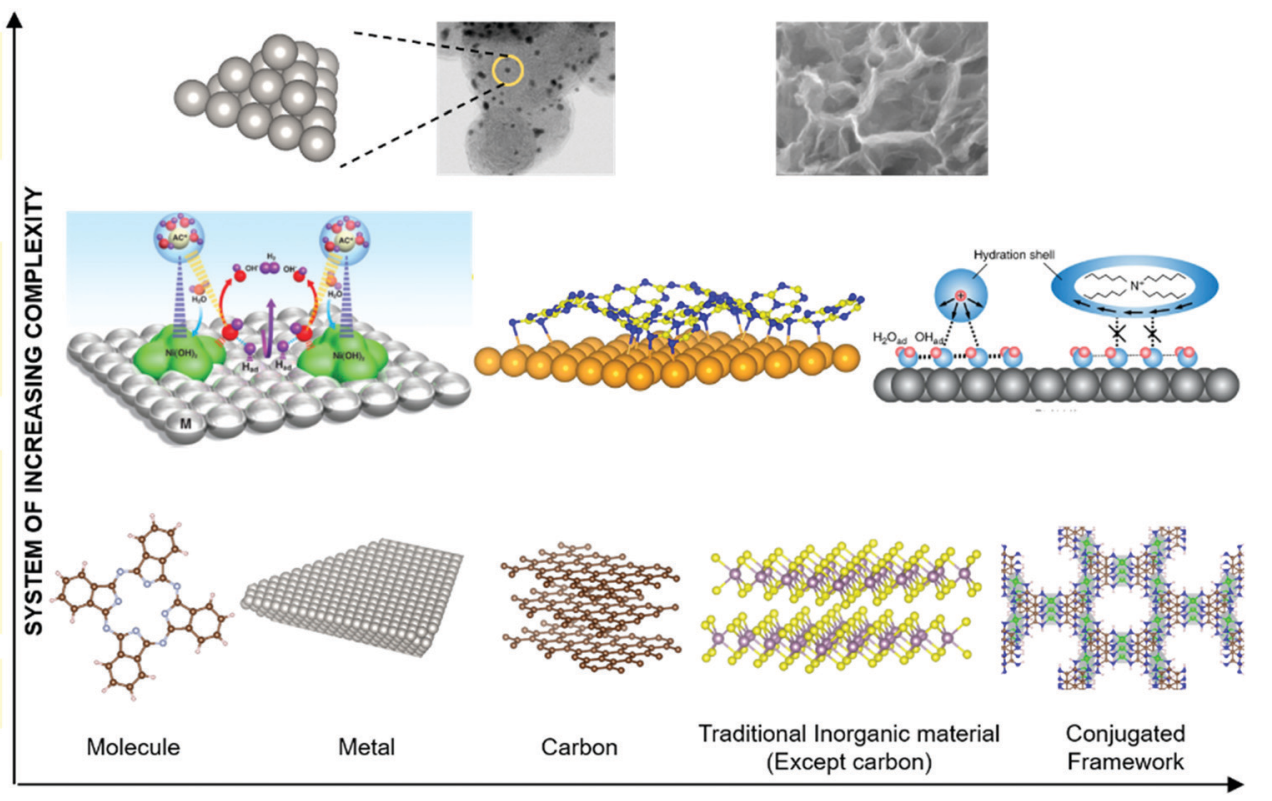

ELECTRODE MATERIAL OF INCREASING COMPLEXITY

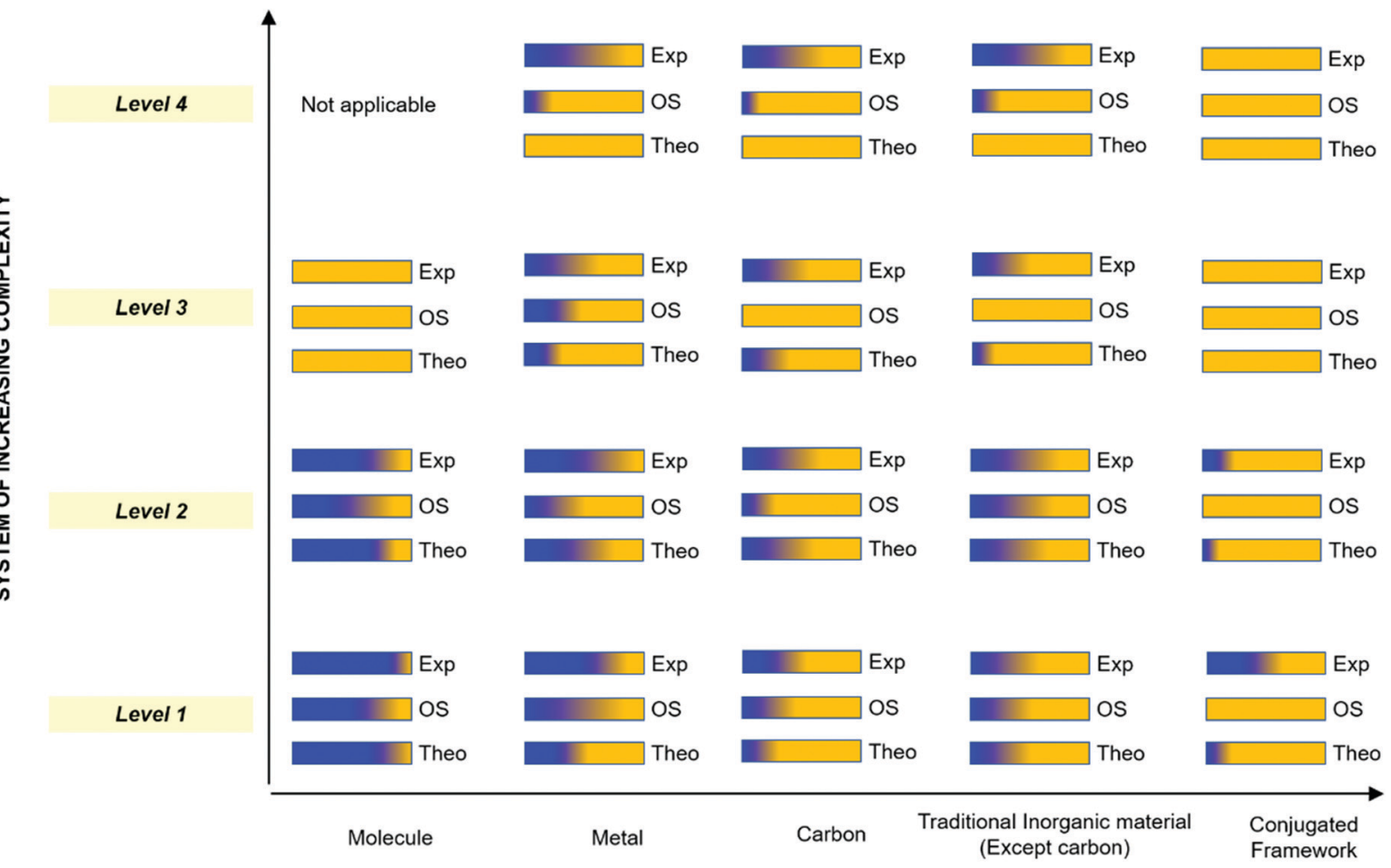

ELECTRODE MATERIAL OF INCREASING COMPLEXITY

Fig. 23 Schematic representation for increasing complexity of model solid-liquid systems as functions of increasing complexity of electrode materials to capture the essential features of a working catalyst. (A) System complexity versus electrode materials complexity diagram. The complexity of model electrode materials increases from metal (generally the simplest) to conjugated frameworks, such as MOFs/COFs, (generally the most complicated). The complexity of model solid/liquid systems increase from Level 2 (alloying, doping, nanostructuring, or other modifications), ${ }^{398-439}$ Level 3 (addition of interfacial promotors on electrode surfaces and/or in electrolyte), ${ }^{221,440-444}$ to Level $4 .^{445-453}$ VESTA was used to prepare several structure images in the panel A. ${ }^{454}$ (B) Evaluations of present level of achievement for each combination of an electrode-system complexity. Each level is evaluated with respect to standard electrochemical experiment (Exp), $25,87,337,455-466$ operando surface-electrochemical spectrometry, microscopy or other analytical methods (OS), ${ }^{467-495}$ and theory (Theo), ${ }^{64,65,107,351,352,386,496-508}$ and with respect to progress that has been made to achieve a proper representation/description of each level for basic multi-electro/-proton transfer electrode processes, for instance, HER, HOR, ORR and CO oxidation reaction. The evaluation was made based on the discussions and references in this Perspective Review. Blue: yes, achieved; orange: no, not achieved. This figure was prepared by referencing the schematic and pictorial representation of model solid/gas systems as a function of increasing complexity to capture the essential features of a working catalyst. ${ }^{49}$ The panel A is partially adopted from the ref. 443, Copyright (2011), with permission from American Association for the Advancement of Science, the ref. 105, Copyright (2017), with permission from American Chemical Society, and the ref. 221. 
solid-liquid interfaces present additional challenges, particularly in terms of the EDL, as discussed in this review.

Although we still need basic understanding about electrocatalytic reactions at single crystals, operando electrochemical techniques have already been applied to investigate these reactions not only for Pt nanoparticles and nanostructured $\mathrm{MoS}_{2},{ }^{509-512}$ but also for metal-free carbon catalysts. ${ }^{513}$ Interestingly, most material systems with realistic structures and/or morphologies (Level 4 in Fig. 23) have already been studied to evaluate their electrochemical properties, possibly because of recent interest in electrochemical energy devices. However, it was clarified that fundamental investigations before going to Level 4 are lacking. This indicated that fundamental research of Levels 1-3 will be necessary to bring the reliable impact at Level 4 (see Fig. 23A the descriptions about these levels). This is especially necessary for carbon-based catalysts, where materials are very sensitive to impurities and/or exhibit complicated doping effects. ${ }^{514-517}$ Therefore, fundamental studies are very important to uncover the complicated characteristics of this catalyst system. ${ }^{518-521}$ The electrode process science at solid-liquid interfaces has another advantage in being able to incorporate ongoing advanced technology, such as operando spectrometries using $\mathrm{X}$-rays at ambient conditions, i.e. X-ray free electron laser and resonance inelastic X-ray scattering, ${ }^{522-527}$ or other surface sensitive operando spectrometries such as two-dimensional heterodynedetected vibrational sum-frequency generation ${ }^{239,528}$ and advanced scanning probe microscopies. ${ }^{529-533}$

Computational studies have also benefitted greatly from approaches developed to study solid-gas interfaces. However, most computational electrocatalysis studies have treated the electrochemical interface using approaches adopted from computational surface science and heterogeneous catalysis without self-consistently accounting for the liquid electrolyte nor the electrode potential, the defining factors of an electrochemical system. With recent advances in theory and computational implementations, both the liquid environment and the electrode potential can now be treated self-consistently from first principles as discussed in Sections 6 and 7. In particular, the electrode potential can be included using GCE-DFT. The liquid environment is, in fact, more challenging, and several approaches exist. The most primitive models based on dielectric continuum theory are being replaced by more refined models from statistical theory of liquids using RISM or classical DFT. ${ }^{334,535}$

With advances in modelling the electrochemical interface, the computational community is also moving from thermodynamics to addressing electrocatalytic and electrochemical kinetics. Thus far, the kinetics have been addressed for innersphere reactions using classical, adiabatic harmonic TST using tools adopted from computational surface science and heterogeneous catalysis. While classical inner-sphere reactions are important for some catalytic reactions, there are many reactions that display outer-sphere character, non-adiabaticity, and/or nuclear tunneling. In addition to accounting for these reaction mechanisms, approaches beyond the harmonic approximation are needed to describe kinetics at strongly anharmonic solid-liquid interfaces. Another open question relates to the valid reaction coordinate; approaches developed for solid-gas interfaces use bond distances as reaction coordinates, while electrochemical rate theories (i.e., Marcus, Newns-AndersonSchmickler, or Soudackov-Hammes-Schiffer approaches) are based on the environment reorganization as the reaction coordinate. The reorganization coordinate used in the general GCE-RT ${ }^{66}$ by combining GCE-DFT with constrained DFT ${ }^{328,329}$ has been demonstrated to give reliable estimates of electron/ hole transfer in solid battery materials. ${ }^{536}$ Combining first principles methods with GCE-RT outlined in Section 6, as well as the (non-adiabatic) quantum or semiclassical dynamics, present promising approaches to address electrochemical kinetics beyond classical harmonic TST and the inner-sphere reaction coordinate. These approaches are also derived from exact rate theory and quantum dynamics, making them suitable for welldefined approximations to address quantum effects, solvent reorganization and stabilization, and different reaction mechanisms in electrochemical processes.

Combinations of these techniques, as well as the analytical methods/theories described in Section 5, will bring a clear picture of real-time reaction dynamics at electrochemical solid-liquid interfaces. As described in Fig. 23, the general understanding of electrochemical solid-liquid systems is still in a preliminary phase compared to the level for solid-gas systems. An example to describe the present stage of electrochemical solid-liquid interfaces is that we still do not understand the microscopic mechanism of HER at Pt(111), one of the simplest multi-electron/-proton transfer reactions, the complexity of which can be comparable to the $\mathrm{CO}$ oxidation reaction at $\mathrm{Pt}(110)$ in UHV solid-gas systems. ${ }^{537-542}$ Therefore, we still need to use the above mentioned advanced theoretical and experimental techniques to study very basic model systems, i.e. single crystal surfaces. Although nowadays there is a wide spectrum of "advanced" materials for electrocatalysis, the "classical" single crystal electrodes are now even more important than before to advance the field of electrode process science. ${ }^{467,543-568}$ In these above works, the close collaborations between theory and experiment are key, as already well discussed. ${ }^{362,398,569-574}$ We hope that these fundamental investigations will bring knowledge to enable the study of more complicated model systems, such as MOFs/COFs and model electrocatalysts having porous structures or heterojunctions (Fig. 12) or much more realistic structures/morphologies. ${ }^{45,446,575-578}$ of course, there are much more complicated systems, such as microbes. ${ }^{579-588}$ These systems are so complicated that it is challenging to unveil their microscopic working principles. However, after acquiring the fundamental knowledge from the above systems at several levels of complexity, there will be a possibility to uncover the microscopic reaction mechanisms in these more complex systems and to develop highly efficient electrochemical energy technology by taking advantages of quantum phenomena.

In summary, we note here the key issues required to bring the present level of understanding of quantum electrochemistry at solid-liquid systems into the next level of complexity: 
(1) An advanced experimental approach to indentify a variety of quantum effects in electrode processes, such as vibronically non-adiabatic tunneling, is essential. This issue is inseparable from issue (2) given below.

(2) Theoretical and computational methods should be further developed and applied to understand fundamental electrode processes and to provide experimentally verifiable predictions. In particular, comparisons of KIEs and spectroscopies between theory and experiment will provide significant insights into the microscopic mechanisms and the important fundamental interactions at solid-liquid interfaces.

(3) Understanding the dynamic interfacial evolution effects for quantum electrode processes by combinations of experimental and theoretical studies. For example, geometries of electrified surfaces ${ }^{589,590}$ and water orientations are dynamically changed by the applied potential. Including these dynamical effects in our interpretation of experimental data and theoretical calculations will enable us to understand complex quantum phenomena in electrode processes.

\section{Conflicts of interest}

The authors have no conflict to declare.

\section{Acknowledgements}

KS is thankful for MMM and SHS for their fruitful discussions, comments and modifications for Sections 1-3 and 8. KS and TK are indebted to the supports from National Institute for Materials Science for fundamental science. This work was partially supported by JSPS KAKENHI grant numbers 19K15527 and 19H05460, and TIA collaborative research program [Kakehashi] Program No. TK19-002 and TK20-11. MMM acknowledges financial support by the Academy of Finland (project 307853) and computational resources provided by CSC - IT Services for Science. MMM also thanks Georg Kastlunger and Per Lindgren for providing the vibrational frequencies for Pt-catalyzed HER. SHS acknowledges financial support from the Center for Alkaline-Based Energy Solutions, an Energy Frontier Research Center, funded by the U.S. Department of Energy, Office of Science, Office of Basic Energy Sciences as well as helpful discussions with Alexander Soudackov and Yan-Choi Lam.

\section{References}

1 D. Keilin, Proc. R. Soc. London, Ser. B, 1925, 98, 312-339.

2 D. Keilin, Proc. R. Soc. London, Ser. B, 1929, 104, 206-252.

3 P. Mitchell, Nature, 1961, 191, 144-148.

4 P. Mitchell, Presented in part at the Nobel Lecture, Stockholm Concert Hall, 1978.

5 X. Jiang, N. Ermolova, J. Lim, S. W. Choi and H. R. Kaback, Proc. Natl. Acad. Sci. U. S. A., 2020, 117, 977-981.

6 J. W. Woodbury, S. H. White, M. C. Mackey, W. L. Hardy and D. B. Chang, in Physical Chemistry An Advanced Treatise, ed. H. Eyring, D. Henderson and W. Jost, Academic Press, New York, USA, 1970, ch. 11, vol. IXB, pp. 904-983.

7 D. M. Dražić, in MTP International Review of Science Physical Chemistry Series One, ed. A. D. Buckingham, Electrochmistry, Butterworths, UK, 1973, vol. 6, pp. 287-332.

8 Y. Cha, C. Murray and J. Klinman, Science, 1989, 243, 1325-1330.

9 B. J. Bahnson, T. D. Colby, J. K. Chin, B. M. Goldstein and J. P. Klinman, Proc. Natl. Acad. Sci. U. S. A., 1997, 94, 12797-12802.

10 A. Kohen, R. Cannio, S. Bartolucci, J. P. Klinman and J. P. Klinman, Nature, 1999, 399, 496-499.

11 K. W. Rickert and J. P. Klinman, Biochemistry, 1999, 38, 12218-12228.

12 M. J. Knapp, K. Rickert and J. P. Klinman, J. Am. Chem. Soc., 2002, 124, 3865-3874.

13 J. P. Klinman and A. R. Offenbacher, Acc. Chem. Res., 2018, 51, 1966-1974.

14 P. R. Schreiner, H. P. Reisenauer, F. C. Pickard Iv, A. C. Simmonett, W. D. Allen, E. Mátyus and A. G. Császár, Nature, 2008, 453, 906-909.

15 P. R. Schreiner, H. P. Reisenauer, D. Ley, D. Gerbig, C.-H. Wu and W. D. Allen, Science, 2011, 332, 1300-1303.

16 P. R. Schreiner, J. Am. Chem. Soc., 2017, 139, 15276-15283.

17 R. W. Gurney, Proc. R. Soc. London, Ser. A, 1931, 134, 137-154.

18 C. E. H. Bawn and G. Ogden, Trans. Faraday Soc., 1934, 30, 432-443.

19 J. Horiuti and M. Polanyi, Acta Physicochim. URSS, 1935, 2, 505-532.

20 J. A. V. Butler, Proc. R. Soc. London, Ser. A, 1936, 157, 423-433.

21 W. F. Libby, J. Phys. Chem., 1952, 56, 863-868.

22 J. Weiss, Proc. R. Soc. London, Ser. A, 1954, 222, 128-141.

23 V. Levich and R. Dogonadze, Dokl. Akad. Nauk SSSR, 1959, 124, 123-126.

24 R. Dogonadze, A. Kuznetsov and V. Levich, Elektrokhimiya, 1967, 3, 739-742.

25 A. N. Frumkin, V. S. Bagotskii, Z. A. Iofa and B. N. Kobanov, Kinetics of Electrode Processes, Moscow State University, Moscow, USSR, 1967.

26 J. Horiuti, in Physical Chemistry An Advanced Treatise, ed. H. Eyring, D. Henderson and W. Jost, Academic Press, New York, USA, 1970, ch. 6, vol. IXB, pp. 543-610.

27 L. G. Levich, in Physical Chemistry An Advanced Treatise, ed. H. Eyring, D. Henderson and W. Jost, Academic Press, New York, USA, 1970, ch. 12, vol. IXB, pp. 985-1074.

28 R. R. Dogonadze, in Reactions of Molecules at Electrodes, ed. N. S. Hush, John Wiley \& Sons Ltd., Bath, UK, 1971, ch. 3, pp. 135-227.

29 A. J. Appleby, J. O. M. Bockris, R. K. Sen and B. E. Conway, in MTP International Review of Science Physical Chemistry Series One, ed. A. D. Buckingham, Electrochemistry, Butterworths, UK, 1973, vol. 6, pp. 1-40.

30 J. O. M. Bockris and S. U. Khan, Quantum Electrochemistry, Plenum Press, USA, 1979. 
31 A. M. Kuznetsov, Electrochim. Acta, 1987, 32, 1271-1278.

32 A. Volta, Philos. Trans. R. Soc. London, 1800, 90, 403-431.

33 M. Faraday, Philos. Trans. R. Soc. London, 1832, 122, 125-162.

34 R. W. Gurney and E. U. Condon, Phys. Rev., 1929, 33, 127-140.

35 R. W. Gurney and P. M. Morse, Phys. Rev., 1929, 33, 789-799.

36 H. Gerischer, Z. Phys. Chem., 1960, 26, 223-247.

37 H. Gerischer, Z. Phys. Chem., 1960, 26, 325-338.

38 H. Gerischer, Z. Phys. Chem., 1961, 27, 48-79.

39 R. A. Marcus, J. Chem. Phys., 1956, 24, 966-978.

40 R. Dogonadze, Y. A. Chizmadzhev and A. Kuznetsov, Zh. Fiz. Khim., 1964, 38, 1194-1202.

41 R. R. Dogonadze, A. M. Kuznetsov and V. G. Levich, Electrochim. Acta, 1968, 13, 1025-1044.

42 W. Schmickler, Ber. Bunsen-Ges., 1973, 77, 991-994.

43 W. Schmickler and W. Vielstich, Electrochim. Acta, 1973, 18, 883-888.

44 R. A. Marcus, Presented in part at the Nobel Lecture, Stockholm Concert Hall, 1992.

45 J. B. Goodenough, Presented in part at the Nobel Lecture, Stockholm Concert Hall, 2019.

46 K. Sakaushi, Faraday Discuss., 2020, 221, 428-448.

47 K. Sakaushi, A. Lyalin and T. Taketsugu, Curr. Opin. Electrochem., 2020, 19, 96-105.

48 X. Lin, N. Nilius, M. Sterrer, P. Koskinen, H. Häkkinen and H.-J. Freund, Phys. Rev. B: Condens. Matter Mater. Phys., 2010, 81, 153406.

49 J. Sauer and H.-J. Freund, Catal. Lett., 2015, 145, 109-125.

50 S. Schauermann and H.-J. Freund, Acc. Chem. Res., 2015, 48, 2775-2782.

51 H.-J. Freund, J. Am. Chem. Soc., 2016, 138, 8985-8996.

52 H.-J. Freund, Rendiconti Lincei, 2017, 28, 5-18.

53 A. Soudackov and S. Hammes-Schiffer, J. Chem. Phys., 1999, 111, 4672-4687.

54 A. Soudackov and S. Hammes-Schiffer, J. Chem. Phys., 2000, 113, 2385-2396.

55 S. Hammes-Schiffer, Acc. Chem. Res., 2001, 34, 273-281.

56 J. Grimminger, S. Bartenschlager and W. Schmickler, Chem. Phys. Lett., 2005, 416, 316-320.

57 M. Otani and O. Sugino, Phys. Rev. B: Condens. Matter Mater. Phys., 2006, 73, 115407.

58 J. Grimminger and W. Schmickler, Chem. Phys., 2007, 334, 8-17.

59 M. Otani, I. Hamada, O. Sugino, Y. Morikawa, Y. Okamoto and T. Ikeshoji, J. Phys. Soc. Jpn., 2008, 77, 024802.

60 M. Otani, I. Hamada, O. Sugino, Y. Morikawa, Y. Okamoto and T. Ikeshoji, Phys. Chem. Chem. Phys., 2008, 10, 3609-3612.

61 J. Haruyama, T. Ikeshoji and M. Otani, Phys. Rev. Mater., 2018, 2, 095801.

62 M. M. Melander, M. J. Kuisma, T. E. K. Christensen and K. Honkala, J. Chem. Phys., 2019, 150, 041706.

63 O. Sugino, J. Phys. Soc. Jpn., 2020, 89, 051013.

64 G. Kastlunger, P. Lindgren and A. A. Peterson, J. Phys. Chem. C, 2018, 122, 12771-12781.

65 P. Lindgren, G. Kastlunger and A. A. Peterson, ACS Catal., 2020, 10, 121-128.
66 M. M. Melander, J. Electrochem. Soc., 2020, 167, 116518. 67 S. Hammes-Schiffer, Energy Environ. Sci., 2012, 5, 7696-7703.

68 C. Costentin, M. Robert and J.-M. Savéant, Chem. Rev., 2010, 110, PR1-PR40.

69 B. E. Conway, Can. J. Chem., 1959, 37, 178-189.

70 E. Conway Brian, Proc. R. Soc. London, Ser. A, 1960, 256, 128-144.

71 B. E. Conway and M. Salomon, Ber. Bunsen-Ges., 1964, 68, 331-340.

72 B. E. Conway and M. Salomon, Electrochim. Acta, 1964, 9, 1599-1615.

73 B. E. Conway and M. Salomon, J. Chem. Phys., 1964, 41, 3169-3177.

74 M. Salomon and B. E. Conway, Ber. Bunsen-Ges., 1965, 69, 669-674.

75 M. Salomon and B. E. Conway, Discuss. Faraday Soc., 1965, 39, 223-238.

76 J. O. M. Bockris and D. B. Matthews, J. Chem. Phys., 1966, 44, 298-309.

77 R. Parsons, Trans. Faraday Soc., 1951, 47, 1332-1344.

78 K. Sakaushi, M. Eckardt, A. Lyalin, T. Taketsugu, R. J. Behm and K. Uosaki, ACS Catal., 2018, 8, 8162-8176.

79 K. Sakaushi, J. Electroanal. Chem., 2019, 849, 113372.

80 M. Eckardt, K. Sakaushi, A. Lyalin, M. Wassner, N. Hüsing, T. Taketsugu and R. J. Behm, Electrochim. Acta, 2019, 299, 736-748.

81 K. Sakaushi, A. Lyalin, T. Taketsugu and K. Uosaki, Phys. Rev. Lett., 2018, 121, 236001.

82 C. Costentin, Chem. Rev., 2008, 108, 2145-2179.

83 C. Costentin, M. Robert and J.-M. Savéant, Acc. Chem. Res., 2010, 43, 1019-1029.

84 M. T. M. Koper, J. Electroanal. Chem., 2011, 660, 254-260.

85 M. T. M. Koper, Chem. Sci., 2013, 4, 2710-2723.

86 D. S. Gnanamuthu and J. V. Petrocelli, J. Electrochem. Soc., 1967, 114, 1036.

87 J. O. M. Bockris and A. K. N. Reddy, Modern Electrochemistry: An Introduction to an interdisciplinary area, Plenum Press, 1970.

88 M. Dunwell, X. Yang, B. P. Setzler, J. Anibal, Y. Yan and B. Xu, ACS Catal., 2018, 8, 3999-4008.

89 A. Goyal, G. Marcandalli, V. A. Mints and M. T. M. Koper, J. Am. Chem. Soc., 2020, 142, 4154-4161.

90 G. A. Parada, Z. K. Goldsmith, S. Kolmar, B. Pettersson Rimgard, B. Q. Mercado, L. Hammarström, S. HammesSchiffer and J. M. Mayer, Science, 2019, 364, 471-475.

91 M. T. Huynh, S. J. Mora, M. Villalba, M. E. Tejeda-Ferrari, P. A. Liddell, B. R. Cherry, A.-L. Teillout, C. W. Machan, C. P. Kubiak, D. Gust, T. A. Moore, S. Hammes-Schiffer and A. L. Moore, ACS Cent. Sci., 2017, 3, 372-380.

92 K. Sakaushi, Phys. Chem. Chem. Phys., 2020, 22, 11219-11243.

93 Z. K. Goldsmith, Y. C. Lam, A. V. Soudackov and S. HammesSchiffer, J. Am. Chem. Soc., 2018, 141, 1084-1090.

94 Y.-C. Lam, A. V. Soudackov and S. Hammes-Schiffer, J. Phys. Chem. Lett., 2019, 10, 5312-5317.

95 M. N. Jackson and Y. Surendranath, J. Am. Chem. Soc., 2016, 138, 3228-3234. 
96 N. S. Hush, J. Chem. Phys., 1958, 28, 962-972.

97 C. E. D. Chidsey, Science, 1991, 251, 919-922.

98 A. Damjanovic and M. A. Genshaw, Electrochim. Acta, 1970, 15, 1281-1283.

99 T. Shinagawa, A. T. Garcia-Esparza and K. Takanabe, Sci. Rep., 2015, 5, 13801.

100 K. Sakaushi and M. Antonietti, Acc. Chem. Res., 2015, 48, 1591-1600.

101 K. Sakaushi and M. Antonietti, Bull. Chem. Soc. Jpn., 2015, 88, 386-398.

102 K. Sakaushi, T.-P. Fellinger and M. Antonietti, ChemSusChem, 2015, 8, 1156-1160.

103 K. Sakaushi, S. J. Yang, T. P. Fellinger and M. Antonietti, J. Mater. Chem. A, 2015, 3, 11720-11724.

104 K. Sakaushi and K. Uosaki, ChemNanoMat, 2016, 2, 99-103. 105 K. Sakaushi, A. Lyalin, S. Tominaka, T. Taketsugu and K. Uosaki, ACS Nano, 2017, 11, 1770-1779.

106 J. K. Nørskov, T. Bligaard, J. Rossmeisl and C. H. Christensen, Nat. Chem., 2009, 1, 37-46.

107 J. Greeley, T. F. Jaramillo, J. Bonde, I. Chorkendorff and J. K. Nørskov, Nat. Mater., 2006, 5, 909-913.

108 N. Brüniche-Olsen and J. Ulstrup, J. Chem. Soc., Faraday Trans. 1, 1979, 75, 205-226.

109 S. J. Edwards, A. V. Soudackov and S. Hammes-Schiffer, J. Phys. Chem. A, 2009, 113, 2117-2126.

110 A. Bouzid and A. Pasquarello, J. Phys. Chem. Lett., 2018, 9, 1880-1884.

111 A. S. Hall, Y. Yoon, A. Wuttig and Y. Surendranath, J. Am. Chem. Soc., 2015, 137, 14834-14837.

112 Y. Yoon, A. S. Hall and Y. Surendranath, Angew. Chem., Int. Ed., 2016, 55, 15282-15286.

113 T. Kambe, R. Sakamoto, K. Hoshiko, K. Takada, M. Miyachi, J.-H. Ryu, S. Sasaki, J. Kim, K. Nakazato, M. Takata and H. Nishihara, J. Am. Chem. Soc., 2013, 135, 2462-2465.

114 T. Yamada, K. Otsubo, R. Makiura and H. Kitagawa, Chem. Soc. Rev., 2013, 42, 6655-6669.

115 E. M. Miner, T. Fukushima, D. Sheberla, L. Sun, Y. Surendranath and M. Dincă, Nat. Commun., 2016, 7, 10942.

116 D. Sheberla, J. C. Bachman, J. S. Elias, C.-J. Sun, Y. ShaoHorn and M. Dincă, Nat. Mater., 2017, 16, 220-224.

117 T. Kitao, Y. Zhang, S. Kitagawa, B. Wang and T. Uemura, Chem. Soc. Rev., 2017, 46, 3108-3133.

118 R. Dong, T. Zhang and X. Feng, Chem. Rev., 2018, 118, 6189-6235.

119 K. Sakaushi, in Functional Organic and Hybrid Nanostructured Materials, ed. Q. Li, Wiley-VCH Verlag GmbH \& Co. KGaA, Weinheim, Germany, 2018, ch. 11, pp. 419-444, DOI: $10.1002 / 9783527807369 . c h 11$.

120 K. Sakaushi, in Materials Nanoarchitectonics, ed. K. Ariga and M. Ebara, John Wiley and Sons, 2018, ch. 8, pp. 155-164, DOI: $10.1002 / 9783527808311 . c h 8$.

121 K. Wada, K. Sakaushi, S. Sasaki and H. Nishihara, Angew. Chem., Int. Ed., 2018, 57, 8886-8890.

122 M. L. Aubrey, B. M. Wiers, S. C. Andrews, T. Sakurai, S. E. Reyes-Lillo, S. M. Hamed, C.-J. Yu, L. E. Darago,
J. A. Mason, J.-O. Baeg, F. Grandjean, G. J. Long, S. Seki, J. B. Neaton, P. Yang and J. R. Long, Nat. Mater., 2018, 17, 625-632.

123 Z. Liang, C. Qu, W. Guo, R. Zou and Q. Xu, Adv. Mater., 2018, 30, 1702891.

124 N. Hosono and S. Kitagawa, Acc. Chem. Res., 2018, 51, 2437-2446.

125 L. S. Xie, G. Skorupskii and M. Dincă, Chem. Rev., 2020, DOI: 10.1021/acs.chemrev.9b00766.

126 M. Amores, K. Wada, K. Sakaushi and H. Nishihara, J. Phys. Chem. C, 2020, 124, 9215-9224.

127 K. Wada, H. Maeda, T. Tsuji, K. Sakaushi, S. Sasaki and H. Nishihara, Inorg. Chem., 2020, 59, 10604-10610.

128 C.-W. Kung, S. Goswami, I. Hod, T. C. Wang, J. Duan, O. K. Farha and J. T. Hupp, Acc. Chem. Res., 2020, 53, 1187-1195.

129 D.-W. Lim and H. Kitagawa, Chem. Rev., 2020, DOI: 10.1021/acs.chemrev.9b00842.

130 K. Sakaushi, G. Nickerl, F. M. Wisser, D. Nishio-Hamane, E. Hosono, H. Zhou, S. Kaskel and J. Eckert, Angew. Chem., Int. Ed., 2012, 51, 7850-7854.

131 K. Sakaushi, E. Hosono, G. Nickerl, T. Gemming, H. Zhou, S. Kaskel and J. Eckert, Nat. Commun., 2013, 4, 1485.

132 K. Sakaushi, G. Nickerl, H. C. Kandpal, L. Cano-Cortés, T. Gemming, J. Eckert, S. Kaskel and J. van den Brink, J. Phys. Chem. Lett., 2013, 4, 2977-2981.

133 S.-Y. Ding and W. Wang, Chem. Soc. Rev., 2013, 42, 548-568.

134 K. Sakaushi, E. Hosono, G. Nickerl, H. Zhou, S. Kaskel and J. Eckert, J. Power Sources, 2014, 245, 553-556.

135 P. J. Waller, F. Gándara and O. M. Yaghi, Acc. Chem. Res., 2015, 48, 3053-3063.

136 N. Huang, P. Wang and D. Jiang, Nat. Rev. Mater., 2016, $1,16068$.

137 I. Ghosh, J. Khamrai, A. Savateev, N. Shlapakov, M. Antonietti and B. König, Science, 2019, 365, 360-366.

138 X. Li, P. Yadav and K. P. Loh, Chem. Soc. Rev., 2020, 49, 4835-4866.

139 A. Savateev, N. V. Tarakina, V. Strauss, T. Hussain, K. ten Brummelhuis, J. M. Sánchez Vadillo, Y. Markushyna, S. Mazzanti, A. P. Tyutyunnik, R. Walczak, M. Oschatz, D. M. Guldi, A. Karton and M. Antonietti, Angew. Chem., Int. Ed., DOI: 10.1002/anie.202004747.

140 K. Geng, T. He, R. Liu, S. Dalapati, K. T. Tan, Z. Li, S. Tao, Y. Gong, Q. Jiang and D. Jiang, Chem. Rev., 2020, DOI: 10.1021/acs.chemrev.9b00550.

141 J. Bardeen, Phys. Rev., 1947, 71, 717-727.

142 N. Cabrera and N. F. Mott, Rep. Prog. Phys., 1949, 12, 163-184.

143 F. F. Vol'kenshtein, Russ. Chem. Rev., 1966, 35, 537-546.

144 X. Lin, B. Yang, H.-M. Benia, P. Myrach, M. Yulikov, A. Aumer, M. A. Brown, M. Sterrer, O. Bondarchuk, E. Kieseritzky, J. Rocker, T. Risse, H.-J. Gao, N. Nilius and H.-J. Freund, J. Am. Chem. Soc., 2010, 132, 7745-7749.

145 C. Stiehler, F. Calaza, W.-D. Schneider, N. Nilius and H.-J. Freund, Phys. Rev. Lett., 2015, 115, 036804. 
146 C. P. O’Brien, K. H. Dostert, M. Hollerer, C. Stiehler, F. Calaza, S. Schauermann, S. Shaikhutdinov, M. Sterrer and H. J. Freund, Faraday Discuss., 2016, 188, 309-321.

147 F. Calaza, C. Stiehler, Y. Fujimori, M. Sterrer, S. Beeg, M. Ruiz-Oses, N. Nilius, M. Heyde, T. Parviainen, K. Honkala, H. Häkkinen and H.-J. Freund, Angew. Chem., Int. Ed., 2015, 54, 12484-12487.

148 P. Alexa, J. M. Lombardi, P. Abufager, H. F. Busnengo, D. Grumelli, V. S. Vyas, F. Haase, B. V. Lotsch, R. Gutzler and K. Kern, Angew. Chem., Int. Ed., 2020, 59, 8411-8415.

149 C. Costentin, S. Drouet, M. Robert and J.-M. Savéant, Science, 2012, 338, 90-94.

150 C. Costentin, S. Drouet, M. Robert and J.-M. Savéant, J. Am. Chem. Soc., 2012, 134, 11235-11242.

151 C. Costentin, M. Robert and J.-M. Savéant, Acc. Chem. Res., 2015, 48, 2996-3006.

152 M. L. Rigsby, D. J. Wasylenko, M. L. Pegis and J. M. Mayer, J. Am. Chem. Soc., 2015, 137, 4296-4299.

153 I. Azcarate, C. Costentin, M. Robert and J.-M. Savéant, J. Am. Chem. Soc., 2016, 138, 16639-16644.

154 M. L. Pegis, C. F. Wise, B. Koronkiewicz and J. M. Mayer, J. Am. Chem. Soc., 2017, 139, 11000-11003.

155 Y.-H. Wang, M. L. Pegis, J. M. Mayer and S. S. Stahl, J. Am. Chem. Soc., 2017, 139, 16458-16461.

156 M. L. Pegis, D. J. Martin, C. F. Wise, A. C. Brezny, S. I. Johnson, L. E. Johnson, N. Kumar, S. Raugei and J. M. Mayer, J. Am. Chem. Soc., 2019, 141, 8315-8326.

157 D. J. Martin, B. Q. Mercado and J. M. Mayer, Sci. Adv., 2020, 6, eaaz3318.

158 D. J. Martin, C. F. Wise, M. L. Pegis and J. M. Mayer, Acc. Chem. Res., 2020, 53, 1056-1065.

159 C. G. Margarit, N. G. Asimow, C. Costentin and D. G. Nocera, ACS Energy Lett., 2020, 5, 72-78.

160 T. Iwasita and F. C. Nart, Prog. Surf. Sci., 1997, 55, 271-340.

161 W. Chen, A. Yu, Z.-J. Sun, B.-Q. Zhu, J. Cai and Y.-X. Chen, Curr. Opin. Electrochem., 2019, 14, 113-123.

162 F. Kitamura, M. Taiseda, M. Takahashi and M. Ito, Chem. Phys. Lett., 1987, 142, 318-322.

163 S.-C. Chang, L.-W. H. Leung and M. J. Weaver, J. Phys. Chem., 1989, 93, 5341-5345.

164 Y. Kinomoto, S. Watanabe, M. Takahashi and M. Ito, Surf. Sci., 1991, 242, 538-543.

165 E. Morallon, A. Rodes, J. L. Vazquez and J. M. Perez, J. Electroanal. Chem., 1995, 391, 149-157.

166 M. Nakamura, H. Ogasawara, J. Inukai and M. Ito, Surf. Sci., 1993, 283, 248-254.

167 B. O. Budevska and P. R. Griffiths, Anal. Chem., 1993, 65, 2963-2971.

168 M. Osawa, K.-I. Ataka, K. Yoshii and T. Yotsuyanagi, J. Electron Spectrosc. Relat. Phenom., 1993, 64-65, 371-379.

169 G. Samjeske and M. Osawa, Angew. Chem., Int. Ed., 2005, 44, 5694-5698.

170 G. Samjeske, A. Miki and M. Osawa, J. Phys. Chem. C, 2007, 111, 15074-15083.

171 G. Samjeske, A. Miki, S. Ye and M. Osawa, J. Phys. Chem. B, 2006, 110, 16559-16566.
172 G. Samjeske, A. Miki, S. Ye, A. Yamakata, Y. Mukouyama, H. Okamoto and M. Osawa, J. Phys. Chem. B, 2006, 109, 23509-23516.

173 M. Osawa, K. Yoshii, K.-I. Ataka and T. Yotsuyanagi, Langmuir, 1994, 10, 640-642.

174 H. Noda, K.-I. Ataka, L.-J. Wan and M. Osawa, Surf. Sci., 1999, 427-428, 190-194.

175 A. Yamakata and M. Osawa, J. Electroanal. Chem., 2017, 800, 19-24.

176 D. Palecek, G. Tek, J. Lan, M. Iannuzzi and P. Hamm, J. Phys. Chem. Lett., 2018, 9, 1254-1259.

177 H. Abramczyk, B. Brozek-Pluska, K. Kurczewski, M. Kurczewski, I. Szymczyk, P. Krzyczmonik, T. Blaszczyk, H. Scholl and W. Czajkowski, J. Phys. Chem. A, 2006, 110, 8627-8636.

178 R. L. Garrell, Anal. Chem., 1989, 61, 401-411.

179 J. F. Li, Y. F. Huang, Y. Ding, Z. L. Yang, S. B. Li, X. S. Zhou, F. R. Fan, W. Zhang, Z. Y. Zhou, D. Y. Wu, B. Ren, Z. L. Wang and Z. Q. Tian, Nature, 2010, 464, 392-395.

180 C. Costentin, M. Robert and J.-M. Savéant, Chem. Soc. Rev., 2013, 42, 2423-2436.

181 Y.-X. Chen, M. Heinen, Z. Jusys and R. J. Behm, ChemPhysChem, 2007, 8, 380-385.

182 J. Schnaidt, M. Heinen and R. J. Behm, Catal. Today, 2013, 202, 154-162.

183 A. Wuttig, M. Yaguchi, K. Motobayashi, M. Osawa and Y. Surendranath, Proc. Natl. Acad. Sci. U. S. A., 2016, 113, E4585-E4593.

184 H. Lv, D. Li, D. Strmcnik, A. P. Paulikas, N. M. Markovic and V. R. Stamenkovic, Nano Energy, 2016, 29, 149-165.

185 N. M. Markovic, B. N. Grgur and P. N. Ross, J. Phys. Chem. $B$, 1997, 101, 5405-5413.

186 M. R. Generro de Chialvo and A. C. Chialvo, Electrochem. Commun., 1999, 1, 379-382.

187 A. Lasia, Int. J. Hydrogen Energy, 2019, 44, 19484-19518.

188 K. Kunimatsu, T. Senzaki, M. Tsushima and M. Osawa, Chem. Phys. Lett., 2005, 401, 451-454.

189 K. Kunimatsu, T. Senzaki, G. Samjeské, M. Tsushima and M. Osawa, Electrochim. Acta, 2007, 52, 5715-5724.

190 M. Nakamura, T. Kobayashi and N. Hoshi, Surf. Sci., 2011, 605, 1462-1465.

191 Ş. C. Bădescu, K. Jacobi, Y. Wang, K. Bedürftig, G. Ertl, P. Salo, T. Ala-Nissila and S. C. Ying, Phys. Rev. B: Condens. Matter Mater. Phys., 2003, 68, 205401.

192 L. Yan, Y. Sun, Y. Yamamoto, S. Kasamatsu, I. Hamada and O. Sugino, J. Chem. Phys., 2018, 149, 164702.

193 A. Peremans and A. Tadjeddine, J. Chem. Phys., 1995, 103, 7197. 194 J. K. Nørskov, J. Rossmeisl, A. Logadottir, L. Lindqvist, J. R. Kitchin, T. Bligaard and H. Jonsson, J. Phys. Chem. B, 2004, 108, 17886-17892.

195 M. H. Shao and R. R. Adzic, J. Phys. Chem. B, 2005, 109, 16563-16566.

196 R. R. Adzic, N. M. Markovic and V. B. Vesovic, J. Electroanal. Chem., 1984, 165, 105-120.

197 S. Strbac, N. A. Anastasijevic and R. R. Adzic, J. Electroanal. Chem., 1992, 323, 179-195. 
198 H. Y. Yu, X. F. Li, T. H. Zhang, J. Liu, J. H. Tian and R. Yang, ChemSusChem, 2020, 13, 2702-2708.

199 X. Li and A. A. Gewirth, J. Am. Chem. Soc., 2005, 127, 5252-5260.

200 M.-h. Shao, P. Liu and R. R. Adzic, J. Am. Chem. Soc., 2006, 128, 7408-7409.

201 S. Nayak, I. J. McPherson and K. A. Vincent, Angew. Chem., Int. Ed., 2018, 57, 12855-12858.

202 J.-C. Dong, X.-G. Zhang, V. Briega-Martos, X. Jin, J. Yang, S. Chen, Z.-L. Yang, D.-Y. Wu, J. M. Feliu, C. T. Williams, Z.-Q. Tian and J.-F. Li, Nat. Energy, 2018, 4, 60-67.

203 J. C. Dong, M. Su, V. Briega-Martos, L. Li, J. B. Le, P. Radjenovic, X. S. Zhou, J. M. Feliu, Z. Q. Tian and J. F. Li, J. Am. Chem. Soc., 2020, 142, 715-719.

204 N. M. Markovic, H. A. Gasteiger and P. N. Ross, J. Phys. Chem., 1995, 99, 3411-3415.

205 J. X. Wang, N. M. Markovic and R. R. Adzic, J. Phys. Chem. $B$, 2004, 108, 4127-4133.

206 J. Tymoczko, V. Colic, A. Ganassin, W. Schuhmann and A. S. Bandarenka, Catal. Today, 2015, 244, 96-102.

207 H. Ogasawara, Y. Sawatari, J. Inukai and M. Ito, J. Electroanal. Chem., 1993, 358, 337-342.

208 Y. Sawatari, J. Inukai and M. Ito, J. Electron Spectrosc. Relat. Phenom., 1993, 64, 515-522.

209 K. Kodama, A. Shinohara, N. Hasegawa, K. Shinozaki, R. Jinnouchi, T. Suzuki, T. Hatanaka and Y. Morimoto, J. Electrochem. Soc., 2014, 161, F649-F652.

210 J. Tymoczko, F. Calle-Vallejo, V. Colic, M. T. M. Koper, W. Schuhmann and A. S. Bandarenka, ACS Catal., 2014, 4, 3772-3778.

211 K. Kodama, K. Motobayashi, A. Shinohara, N. Hasegawa, K. Kudo, R. Jinnouchi, M. Osawa and Y. Morimoto, ACS Catal., 2018, 8, 694-700.

212 A. M. Gómez-Marín, A. Berná and J. M. Feliu, J. Phys. Chem. C, 2010, 114, 20130-20140.

213 N. M. Markovic, R. R. Adzic, B. D. Cahan and E. B. Yeager, J. Electroanal. Chem., 1994, 377, 249-259.

214 H. Tanaka, S. Sugawara, K. Shinohara, T. Ueno, S. Suzuki, N. Hoshi and M. Nakamura, Electrocatalysis, 2015, 6, 295-299.

215 T. Ueno, H. Tanaka, S. Sugawara, K. Shinohara, A. Ohma, N. Hoshi and M. Nakamura, J. Electroanal. Chem., 2017, 800, 162-166.

216 F. Zaera, Prog. Surf. Sci., 2001, 69, 1-98.

217 D. Strmcnik, K. Kodama, D. van der Vliet, J. Greeley, V. R. Stamenkovic and N. M. Markovic, Nat. Chem., 2009, 1, 466-472.

218 M. Nakamura, Y. Nakajima, N. Hoshi, H. Tajiri and O. Sakata, ChemPhysChem, 2013, 14, 2426-2431.

219 A. Yamakata and M. Osawa, J. Phys. Chem. Lett., 2010, 1, 1487-1491.

220 T. Kumeda, R. Kubo, N. Hoshi and M. Nakamura, ACS Appl. Energy Mater., 2019, 2, 3904-3909.

221 T. Kumeda, H. Tajiri, O. Sakata, N. Hoshi and M. Nakamura, Nat. Commun., 2018, 9, 4378.

222 S. Yamamoto, A. Beniya, K. Mukai, Y. Yamashita and J. Yoshinobu, J. Phys. Chem. B, 2005, 109, 5816-5823.
223 N. Ramaswamy and S. Mukerjee, J. Phys. Chem. C, 2011, 115, 18015-18026.

224 S. Chatterjee, K. Sengupta, S. Samanta, P. K. Das and A. Dey, Inorg. Chem., 2015, 54, 2383-2392.

225 C. Zou, M. Larisika, G. Nagy, J. Srajer, C. Oostenbrink, X. Chen, W. Knoll, B. Liedberg and C. Nowak, J. Phys. Chem. B, 2013, 117, 9606-9614.

226 S. Chatterjee, K. Sengupta, S. Samanta, P. K. Das and A. Dey, Inorg. Chem., 2013, 52, 9897-9907.

227 M. Grosserueschkamp, M. G. Friedrich, M. Plum, W. Knoll and R. L. C. Naumann, J. Phys. Chem. B, 2009, 113, 2492-2497.

228 C. Nowak, C. Luening, D. Schach, D. Baurecht, W. Knoll and R. L. C. Naumann, J. Phys. Chem. C, 2009, 113, 2256-2262.

229 A. Schwaighofer, C. Steininger, D. M. Hildenbrandt, J. Srajer, C. Nowak, W. Knoll and R. L. C. Naumann, Biophys. J., 2013, 105, 2706-2713.

230 H. K. Ly, P. Wrzolek, N. Heidary, R. Gotz, M. Horch, J. Kozuch, M. Schwalbe and I. M. Weidinger, Chem. Sci., 2015, 6, 6999-7007.

231 T. Chen, Z. Feng, G. Wu, J. Shi, G. Ma, P. Ying and C. Li, J. Phys. Chem. C, 2007, 111, 8005-8014.

232 S. Zhu, T. Li, W.-B. Cai and M. Shao, ACS Energy Lett., 2019, 4, 682-689.

233 J. Heyes, M. Dunwell and B. Xu, J. Phys. Chem. C, 2016, 120, 17334-17341.

234 M. Dunwell, Q. Lu, J. M. Heyes, J. Rosen, J. G. Chen, Y. Yan, F. Jiao and B. Xu, J. Am. Chem. Soc., 2017, 139, 3774-3783.

235 Q. Cheng, L. Wei, Z. Liu, N. Ni, Z. Sang, B. Zhu, W. Xu, M. Chen, Y. Miao, L.-Q. Chen, W. Min and Y. Yang, Nat. Commun., 2018, 9, 2942.

236 P. Guyot-Sionnest and A. Tadjeddine, Chem. Phys. Lett., 1990, 172, 341-345.

237 A. Peremans and A. Tadjeddine, Phys. Rev. Lett., 1994, 73, 3010-3013.

238 W. Zhou, S. Inoue, T. Iwahashi, K. Kanai, K. Seki, T. Miyamae, D. Kim, Y. Katayama and Y. Ouchi, Electrochem. Commun., 2010, 12, 672-675.

239 A. Sayama, S. Nihonyanagi, Y. Ohshima and T. Tahara, Phys. Chem. Chem. Phys., 2020, 22, 2580-2589.

240 X. Wang, S.-C. Huang, T.-X. Huang, H.-S. Su, J.-H. Zhong, Z.-C. Zeng, M.-H. Lia and B. Ren, Chem. Soc. Rev., 2017, 46, 4020-4041.

241 A. Soudackov and S. Hammes-Schiffer, J. Chem. Phys., 1999, 111, 4672-4687.

242 A. Soudackov and S. Hammes-Schiffer, J. Chem. Phys., 2000, 113, 2385-2396.

243 S. Hammes-Schiffer and A. V. Soudackov, J. Phys. Chem. B, 2008, 112, 14108-14123.

244 S. Hammes-Schiffer and A. A. Stuchebrukhov, Chem. Rev., 2010, 110, 6939-6960.

245 Y. Georgievskii and A. A. Stuchebrukhov, J. Chem. Phys., 2000, 113, 10438-10450.

246 J. H. Skone, A. V. Soudackov and S. Hammes-Schiffer, J. Am. Chem. Soc., 2006, 128, 16655-16663. 
247 D. F. Calef and P. G. Wolynes, J. Phys. Chem., 1983, 87, 3387-3400.

248 J. T. Hynes, J. Phys. Chem., 1986, 90, 3701-3706.

249 J. E. Straub and B. J. Berne, J. Chem. Phys., 1987, 87, 6111-6116.

250 D. E. Makarov and M. Topaler, Chem. Phys. Lett., 1995, 245, 343-350.

251 C. Venkataraman, A. V. Soudackov and S. HammesSchiffer, J. Phys. Chem. C, 2008, 112, 12386-12397.

252 I. Navrotskaya, A. V. Soudackov and S. Hammes-Schiffer, J. Chem. Phys., 2008, 128, 244712.

253 B. Auer, L. E. Fernandez and S. Hammes-Schiffer, J. Am. Chem. Soc., 2011, 133, 8282-8292.

254 M. T. Huynh, S. J. Mora, M. Villalba, M. E. Tejeda-Ferrari, P. A. Liddell, B. R. Cherry, A.-L. Teillout, C. W. Machan, C. P. Kubiak and D. Gust, ACS Cent. Sci., 2017, 3, 372-380.

255 Y.-C. Lam, A. V. Soudackov, Z. K. Goldsmith and S. HammesSchiffer, J. Phys. Chem. C, 2019, 123, 12335-12345.

256 G. Guoy, J. Phys., 1910, 9, 457-467.

257 D. L. Chapman, London Edinburgh Philos. Mag. J. Sci., 1913, 25, 475-481.

258 O. Stern, Z. Elektrochem., 1924, 30, 1014-1020.

259 A. Velikonja, E. Gongadze, V. Kralj-Iglic and A. Iglic, Int. J. Electrochem. Sci., 2014, 9.

260 Z. K. Goldsmith, M. Secor and S. Hammes-Schiffer, ACS Cent. Sci., 2020, 6, 304-311.

261 A. Hamelin and M. J. Weaver, J. Electroanal. Chem. Interfacial Electrochem., 1987, 223, 171-184.

262 B. E. Conway, Proc. R. Soc. London, Ser. A, 1960, 256, 128-144.

263 Z.-D. He, Y.-X. Chen, E. Santos and W. Schmickler, Angew. Chem., Int. Ed., 2018, 57, 7948-7956.

264 W. H. Miller, J. Chem. Phys., 1974, 61, 1823-1834.

265 R. Sundararaman, W. A. Goddard III and T. A. Arias, J. Chem. Phys., 2017, 146, 114104.

266 J. D. Goodpaster, A. T. Bell and M. Head-Gordon, J. Phys. Chem. Lett., 2016, 7, 1471-1477.

267 N. Bonnet, T. Morishita, O. Sugino and M. Otani, Phys. Rev. Lett., 2012, 109, 266101.

268 R. Jinnouchi, K. Kodama and Y. Morimoto, Curr. Opin. Electrochem., 2018, 8, 103-109.

269 W. H. Miller, S. D. Schwartz and J. W. Tromp, J. Chem. Phys., 1983, 79, 4889-4898.

270 W. H. Miller, J. Phys. Chem. A, 1998, 102, 793-806.

271 F. Studt, J. K. Nørskov, F. Abild-Pedersen and T. Bligaard, Fundamental concepts in heterogeneous catalysis, John Wiley \& Sons, Inc., 2014.

272 Y. Huang, R. J. Nielsen and W. A. Goddard, J. Am. Chem. Soc., 2018, 140, 16773-16782.

273 K. Chan and J. K. Nørskov, J. Phys. Chem. Lett., 2016, 7, 1686-1690.

274 M. J. Janik and M. Neurock, Electrochim. Acta, 2007, 52, 5517-5528.

275 M. Van den Bossche, E. Skúlason, C. Rose-Petruck and H. Jónsson, J. Phys. Chem. C, 2019, 123, 4116-4124.

276 G. Henkelman, B. P. Uberuaga and H. Jónsson, J. Chem. Phys., 2000, 113, 9901-9904.
277 T. Ikeshoji and M. Otani, Phys. Chem. Chem. Phys., 2017, 19, 4447-4453.

278 R. Kronberg, H. Lappalainen and K. Laasonen, Phys. Chem. Chem. Phys., 2020, 22, 10536-10549.

279 M. Sprik and G. Ciccotti, J. Chem. Phys., 1998, 109, 7737-7744.

280 A. J. Garza, A. T. Bell and M. Head-Gordon, ACS Catal., 2018, 8, 1490-1499.

281 H. Xiao, T. Cheng, W. A. Goddard and R. Sundararaman, J. Am. Chem. Soc., 2016, 138, 483-486.

282 H. S. Pillai and H. Xin, Ind. Eng. Chem. Res., 2019, 58, 10819-10828.

283 L.-Y. Chen, T.-C. Kuo, Z.-S. Hong, M.-J. Cheng and W. A. Goddard, Phys. Chem. Chem. Phys., 2019, 21, 17605-17612.

284 J. E. Lawrence and D. E. Manolopoulos, Faraday Discuss., 2020, 221, 9-29.

285 Y. Zhang, J. B. Rommel, M. T. Cvitå and S. C. Althorpe, Phys. Chem. Chem. Phys., 2014, 16, 24292-24300.

286 J. O. Richardson, PhD thesis, University of Cambridge, 2012.

287 I. Affleck, Phys. Rev. Lett., 1981, 46, 388-391.

288 M. J. Gillan, J. Phys. C: Solid State Phys., 1987, 20, 3621-3641.

289 M. Tuckerman, Statistical Mechanics: Theory and Molecular Simulations, Oxford University Press, 2010.

290 R. P. Feynman, Rev. Mod. Phys., 1948, 20, 367-387.

291 R. P. Feynman and A. R. Hibbs, Quantum Mechanics and Path Integrals, McGraw-Hill, 1965.

292 S. Coleman, The Uses of Instantons, in The Whys of Subnuclear Physics, ed. A. Zichichi, The Subnuclear Series, Springer, Boston, MA, 1979, vol. 15.

293 P. G. Wolynes, J. Chem. Phys., 1987, 87, 6559-6561.

294 M. Ceriotti, W. Fang, P. G. Kusalik, R. H. McKenzie, A. Michaelides, M. A. Morales and T. E. Markland, Chem. Rev., 2016, 116, 7529-7550.

295 W. Fang, J. Chen, Y. Feng, X.-Z. Li and A. Michaelides, Int. Rev. Phys. Chem., 2019, 38, 35-61.

296 J. Lan, V. V. Rybkin and M. Iannuzzi, J. Phys. Chem. Lett., 2020, 11, 3724-3730.

297 W. H. Miller, J. Chem. Phys., 1975, 62, 1899-1906.

298 E. J. Heller, The Semiclassical Way to Dynamics and Spectroscopy, Princeton University Press, 2018.

299 R. Hernandez and W. H. Miller, Chem. Phys. Lett., 1993, 214, 129-136.

300 W. H. Miller, Faraday Discuss. Chem. Soc., 1977, 62, 40-46. 301 A. F. Wagner, J. Phys. Chem. A, 2013, 117, 13089-13100.

302 X. Shan, T. A. H. Burd and D. C. Clary, J. Phys. Chem. A, 2019, 123, 4639-4657.

303 J. T. Fermann and S. Auerbach, J. Chem. Phys., 2000, 112, 6787-6794.

304 K. Heinola and T. Ahlgren, J. Appl. Phys., 2010, 107, 113531.

305 K. Mudiyanselage, M. Trenary and R. J. Meyer, J. Phys. Chem. C, 2007, 111, 7127-7136.

306 S. C. Althorpe and T. J. H. Hele, J. Chem. Phys., 2013, 139, 084115. 
307 T. J. H. Hele and S. C. Althorpe, J. Chem. Phys., 2013, 138, 084108.

308 J. O. Richardson and S. C. Althorpe, J. Chem. Phys., 2009, 131, 214106.

309 J. O. Richardson, J. Chem. Phys., 2016, 144, 114106.

310 J. O. Richardson, Int. Rev. Phys. Chem., 2018, 37, 171-216.

311 W. H. Miller, Faraday Discuss. Chem. Soc., 1977, 62, 40-46.

312 S. Andersson, G. Nyman, A. Arnaldsson, U. Manthe and H. Jónsson, J. Phys. Chem. A, 2009, 113, 4468-4478.

313 V. Àsgeirsson, A. Arnaldsson and H. Jònsson, J. Chem. Phys., 2018, 148, 102334.

314 J. B. Rommel, T. P. M. Goumans and J. Kästner, J. Chem. Theory Comput., 2011, 7, 690-698.

315 G. Laude, D. Calderini, D. P. Tew and J. O. Richardson, Faraday Discuss., 2018, 212, 237-258.

316 M. Kryvohuz, J. Chem. Phys., 2012, 137, 234304.

317 G. Mills, G. K. Schenter, D. E. Makarov and H. Jónsson, Chem. Phys. Lett., 1997, 278, 91-96.

318 W. Fang, J. O. Richardson, J. Chen, X.-Z. Li and A. Michaelides, Phys. Rev. Lett., 2017, 119, 126001.

319 Y. Litman, J. O. Richardson, T. Kumagai and M. Rossi, J. Am. Chem. Soc., 2019, 141, 2526-2534.

320 D. Chandler and P. G. Wolynes, J. Chem. Phys., 1981, 74, 4078-4095.

321 I. R. Craig and D. E. Manolopoulos, J. Chem. Phys., 2004, 121, 3368-3373.

322 I. R. Craig and D. E. Manolopoulos, J. Chem. Phys., 2005, 122, 084106.

323 I. R. Craig and D. E. Manolopoulos, J. Chem. Phys., 2005, 123, 034102.

324 R. Collepardo-Guevara, I. R. Craig and D. E. Manolopoulos, J. Chem. Phys., 2008, 128, 144502.

325 J. S. Kretchmer and T. F. Miller, J. Chem. Phys., 2013, 138, 134109.

326 J. S. Kretchmer and T. F. Miller, Inorg. Chem., 2016, 55, 1022-1031.

327 Y. V. Suleimanov, J. Phys. Chem. C, 2012, 116, 11141-11153.

328 B. Kaduk, T. Kowalczyk and T. Van Voorhis, Chem. Rev., 2012, 112, 321-370.

329 M. Melander, E. Ö. Jónsson, J. J. Mortensen, T. Vegge and J. M. García-Lastra, J. Chem. Theory Comput., 2016, 12, 5367-5378.

330 G. Kastlunger, personal communication.

331 K. Sakaushi, Faraday Discuss., 2020, 221, 428-448.

332 G. A. Voth, D. Chandler and W. H. Miller, J. Chem. Phys., 1989, 91, 7749-7760.

333 S. Habershon, D. E. Manolopoulos, T. E. Markland and T. F. Miller, Annu. Rev. Phys. Chem., 2013, 64, 387-413.

334 E. R. Heller and J. O. Richardson, J. Chem. Phys., 2020, 152, 034106.

335 M. J. Thapa, W. Fang and J. O. Richardson, J. Chem. Phys., 2019, 150, 104107.

336 J. E. Lawrence and D. E. Manolopoulos, J. Chem. Phys., 2019, 151, 244109.

337 J. O. M. Bockris and S. U. Khan, Surface electrochemistry: a molecular level approach, Plenum Press, New York, USA, 1993.
338 N. M. Marković and P. N. Ross, Surf. Sci. Rep., 2002, 45, 117-229.

339 G. Jerkiewicz, Electrocatalysis, 2010, 1, 179-199.

340 V. Krishna, J. Chem. Phys., 2007, 126, 134107.

341 D. Marx and M. Parrinello, Z. Phys. B: Condens. Matter, 1994, 95, 143-144.

342 J. P. Blaizot and H. Orland, Phys. Rev. C: Nucl. Phys., 1981, 24, 1740-1761.

343 J. C. Tully and R. K. Preston, J. Chem. Phys., 1971, 55, 562-572.

344 J. C. Tully, J. Chem. Phys., 1990, 93, 1061-1071.

345 W. Schmickler, Interfacial electrochemistry, Oxford University Press, The United States of America, 1996.

346 E. Santos, P. Quaino and W. Schmickler, Phys. Chem. Chem. Phys., 2012, 14, 11224-11233.

347 S. Tanaka and C.-P. Hsu, J. Chem. Phys., 1999, 111, 11117-11137.

348 K. Nakamura, Y. Nohara, Y. Yosimoto and Y. Nomura, Phys. Rev. B, 2016, 93, 085124.

349 I. Ledezma-Yanez, W. D. Z. Wallace, P. Sebastián-Pascual, V. Climent, J. M. Feliu and M. T. M. Koper, Nat. Energy, 2017, 2, 17031.

350 P. S. Rice, Y. Mao, C. Guo and P. Hu, Phys. Chem. Chem. Phys., 2019, 21, 5932-5940.

351 R. Jinnouchi and A. B. Anderson, J. Phys. Chem. C, 2008, 112, 8747-8750.

352 R. Jinnouchi and A. B. Anderson, Phys. Rev. B: Condens. Matter Mater. Phys., 2008, 77, 245417.

353 G. Fisicaro, L. Genovese, O. Andreussi, N. Marzari and S. Goedecker, J. Chem. Phys., 2016, 144, 014103.

354 D. Chandler and H. C. Andersen, J. Chem. Phys., 1972, 57, 1930-1937.

355 A. Kovalenko and F. Hirata, J. Chem. Phys., 1999, 110, 10095-10112.

356 S. Nishihara and M. Otani, Phys. Rev. B, 2017, 96, 115429.

357 Y. Noguchi, M. Hiyama, M. Shiga, H. Akiyama and O. Sugino, J. Chem. Theory Comput., 2019, 15, 5474-5482.

358 M. J. Eslamibidgoli, J. Huang, T. Kadyk, A. Malek and M. Eikerling, Nano Energy, 2016, 29, 334-361.

359 S. Maheshwari, Y. Li, N. Agrawal and M. J. Janik, in Advances in Catalysis, ed. C. Song, Academic Press, 2018, vol. 63, pp. 117-167.

360 M. J. Eslamibidgoli and M. H. Eikerling, Curr. Opin. Electrochem., 2018, 9, 189-197.

361 A. Groß and S. Sakong, Curr. Opin. Electrochem., 2019, 14, 1-6.

362 O. M. Magnussen and A. Groß, J. Am. Chem. Soc., 2019, 141, 4777-4790.

363 K. Schwarz and R. Sundararaman, Surf. Sci. Rep., 2020, 75, 100492.

364 T. Ikeshoji, M. Otani, I. Hamada and Y. Okamoto, Phys. Chem. Chem. Phys., 2011, 13, 20223-20227.

365 T. Ikeshoji, M. Otani, I. Hamada, O. Sugino, Y. Morikawa, Y. Okamoto, Y. Qian and I. Yagi, AIP Adv., 2012, 2, 032182.

366 Y. Qian, I. Hamada, M. Otani and T. Ikeshoji, Catal. Today, 2013, 202, 163-167. 
367 T. Ikeshoji, T. Uchida, M. Otani and M. Osawa, J. Electroanal. Chem., 2017, 800, 13-18.

368 Y. Ando, Y. Kawamura, T. Ikeshoji and M. Otani, Chem. Phys. Lett., 2014, 612, 240-244.

369 A. Bouzid and A. Pasquarello, J. Chem. Theory Comput., 2017, 13, 1769-1777.

370 A. Bouzid, P. Gono and A. Pasquarello, J. Catal., 2019, 375, 135-139.

371 V. M. Fernandez-Alvarez and M. H. Eikerling, ACS Appl. Mater. Interfaces, 2019, 11, 43774-43780.

372 C. Zhan, M. R. Cerón, S. A. Hawks, M. Otani, B. C. Wood, T. A. Pham, M. Stadermann and P. G. Campbell, Nat. Commun., 2019, 10, 4858.

373 S. Ringe, E. L. Clark, J. Resasco, A. Walton, B. Seger, A. T. Bell and K. Chan, Energy Environ. Sci., 2019, 12, 3001-3014.

374 J. A. Gauthier, C. F. Dickens, H. H. Heenen, S. Vijay, S. Ringe and K. Chan, J. Chem. Theory Comput., 2019, 15, 6895-6906.

375 H. H. Heenen, J. A. Gauthier, H. H. Kristoffersen, T. Ludwig and K. Chan, J. Chem. Phys., 2020, 152, 144703.

376 S. Sakong and A. Groß, J. Chem. Phys., 2018, 149, 084705.

377 T. Cheng, L. Wang, B. V. Merinov and W. A. Goddard, J. Am. Chem. Soc., 2018, 140, 7787-7790.

378 H. H. Kristoffersen, K. Chan, T. Vegge and H. A. Hansen, Chem. Commun., 2020, 56, 427-430.

379 L. Schimka, J. Harl, A. Stroppa, A. Grüneis, M. Marsman, F. Mittendorfer and G. Kresse, Nat. Mater., 2010, 9, 741-744.

380 T. L. Tan, L.-L. Wang, D. D. Johnson and K. Bai, J. Phys. Chem. C, 2013, 117, 22696-22704.

381 T. T. T. Hanh, Y. Takimoto and O. Sugino, Surf. Sci., 2014, 625, 104-111.

382 L. Yan, Y. Yamamoto, M. Shiga and O. Sugino, Phys. Rev. B, 2020, 101, 165414.

383 M. Nishijima, H. Okuyama, N. Takagi, T. Aruga and W. Brenig, Surf. Sci. Rep., 2005, 57, 113-156.

384 L. Yan, Y. Yamamoto and O. Sugino, Phys. Rev. B, 2020, 101, 165414.

385 W. Fang, J. O. Richardson, J. Chen, X.-Z. Li and A. Michaelides, Phys. Rev. Lett., 2017, 119, 126001.

386 E. Skúlason, G. S. Karlberg, J. Rossmeisl, T. Bligaard, J. Greeley, H. Jónsson and J. K. Nørskov, Phys. Chem. Chem. Phys., 2007, 9, 3241-3250.

387 Y. Yamamoto, S. Kasamatsu and O. Sugino, J. Phys. Chem. C, 2019, 123, 19486-19492.

388 A. R. Zeradjanin, J.-P. Grote, G. Polymeros and K. J. J. Mayrhofer, Electroanalysis, 2016, 28, 2256-2269.

389 S. Ringe, H. Oberhofer and K. Reuter, J. Chem. Phys., 2017, 146, 134103.

390 S. Kasamatsu and O. Sugino, J. Phys.: Condens. Matter, 2019, 31, 085901.

391 G. Ertl, Angew. Chem., Int. Ed., 2008, 47, 3524-3535.

392 G. A. Somorjai and Y. Li, Introduction to surface chemistry and catalysis, John Wiley \& Sons, 2010.

393 H.-J. Freund, H. Kuhlenbeck and V. Staemmler, Rep. Prog. Phys., 1996, 59, 283-347.
394 H.-J. Freund and G. Pacchioni, Chem. Soc. Rev., 2008, 37, 2224-2242.

395 S. Schauermann, N. Nilius, S. Shaikhutdinov and H.-J. Freund, Acc. Chem. Res., 2013, 46, 1673-1681.

396 G. Pacchioni and H. Freund, Chem. Rev., 2013, 113, 4035-4072.

397 W. Ostwald, Über Katalyse, Akademische Verlagsgesellschaft, Leipzig, 1923.

398 I. E. L. Stephens, A. S. Bondarenko, U. Grønbjerg, J. Rossmeisl and I. Chorkendorff, Energy Environ. Sci., 2012, 5, 6744-6762.

399 R. R. Adžić and A. R. Despić, J. Chem. Phys., 1974, 61, 3482-3483.

400 R. R. Adžić and A. R. Despić, Z. Phys. Chem., 1975, 98, 95. 401 R. R. Adžić, D. N. Simić, A. R. Despić and D. M. Dražić, J. Electroanal. Chem. Interfacial Electrochem., 1975, 65, 587-601.

402 R. R. Adžić, A. V. Tripković and W. E. O’Grady, Nature, 1982, 296, 137-138.

403 R. R. Adžić, A. V. Tripković and N. M. Marković, J. Electroanal. Chem. Interfacial Electrochem., 1983, 150, 79-88.

404 N. A. Anastasijević, V. Vesović and R. R. Adžić, J. Electroanal. Chem. Interfacial Electrochem., 1987, 229, 305-316.

405 M. Baldauf and D. M. Kolb, J. Phys. Chem., 1996, 100, 11375-11381.

406 L. A. Kibler, A. M. El-Aziz, R. Hoyer and D. M. Kolb, Angew. Chem., Int. Ed., 2005, 44, 2080-2084.

407 V. Stamenkovic, B. S. Mun, K. J. J. Mayrhofer, P. N. Ross, N. M. Markovic, J. Rossmeisl, J. Greeley and J. K. Nørskov, Angew. Chem., Int. Ed., 2006, 45, 2897-2901.

408 T. F. Jaramillo, K. P. Jørgensen, J. Bonde, J. H. Nielsen, S. Horch and I. Chorkendorff, Science, 2007, 317, 100-102.

409 J. Greeley, I. E. L. Stephens, A. S. Bondarenko, T. P. Johansson, H. A. Hansen, T. F. Jaramillo, J. Rossmeisl, I. Chorkendorff and J. K. Nørskov, Nat. Chem., 2009, 1, 552-556.

410 K. Gong, F. Du, Z. Xia, M. Durstock and L. Dai, Science, 2009, 323, 760-764.

411 P. Strasser, S. Koh, T. Anniyev, J. Greeley, K. More, C. Yu, Z. Liu, S. Kaya, D. Nordlund, H. Ogasawara, M. F. Toney and A. Nilsson, Nat. Chem., 2010, 2, 454-460.

412 Y. Bing, H. Liu, L. Zhang, D. Ghosh and J. Zhang, Chem. Soc. Rev., 2010, 39, 2184-2202.

413 R. Ma and T. Sasaki, Adv. Mater., 2010, 22, 5082-5104.

414 I. E. L. Stephens, A. S. Bondarenko, F. J. Perez-Alonso, F. Calle-Vallejo, L. Bech, T. P. Johansson, A. K. Jepsen, R. Frydendal, B. P. Knudsen, J. Rossmeisl and I. Chorkendorff, J. Am. Chem. Soc., 2011, 133, 5485-5491.

415 W. Yang, T.-P. Fellinger and M. Antonietti, J. Am. Chem. Soc., 2011, 133, 206-209.

416 S. J. Yang, T. Kim, J. H. Im, Y. S. Kim, K. Lee, H. Jung and C. R. Park, Chem. Mater., 2012, 24, 464-470.

417 J. Kibsgaard, Z. Chen, B. N. Reinecke and T. F. Jaramillo, Nat. Mater., 2012, 11, 963-969.

418 M. Escudero-Escribano, A. Verdaguer-Casadevall, P. Malacrida, U. Grønbjerg, B. P. Knudsen, A. K. Jepsen, J. Rossmeisl, I. E. L. Stephens and I. Chorkendorff, J. Am. Chem. Soc., 2012, 134, 16476-16479. 
419 J. P. Paraknowitsch and A. Thomas, Energy Environ. Sci., 2013, 6, 2839-2855.

420 M.-R. Gao, Y.-F. Xu, J. Jiang and S.-H. Yu, Chem. Soc. Rev., 2013, 42, 2986-3017.

421 P. Chen, T.-Y. Xiao, Y.-H. Qian, S.-S. Li and S.-H. Yu, Adv. Mater., 2013, 25, 3192-3196.

422 D. Wang, H. L. Xin, R. Hovden, H. Wang, Y. Yu, D. A. Muller, F. J. DiSalvo and H. D. Abruña, Nat. Mater., 2013, 12, 81-87.

423 T. Fukushima, W. Drisdell, J. Yano and Y. Surendranath, J. Am. Chem. Soc., 2015, 137, 10926-10929.

424 S. J. Yang, M. Antonietti and N. Fechler, J. Am. Chem. Soc., 2015, 137, 8269-8273.

425 M.-R. Gao, J.-X. Liang, Y.-R. Zheng, Y.-F. Xu, J. Jiang, Q. Gao, J. Li and S.-H. Yu, Nat. Commun., 2015, 6, 5982.

426 L. Dai, Y. Xue, L. Qu, H.-J. Choi and J.-B. Baek, Chem. Rev., 2015, 115, 4823-4892.

427 M. Escudero-Escribano, P. Malacrida, M. H. Hansen, U. G. Vej-Hansen, A. Velázquez-Palenzuela, V. Tripkovic, J. Schiøtz, J. Rossmeisl, I. E. L. Stephens and I. Chorkendorff, Science, 2016, 352, 73-76.

428 N. D. Ricke, A. T. Murray, J. J. Shepherd, M. G. Welborn, T. Fukushima, T. Van Voorhis and Y. Surendranath, ACS Catal., 2017, 7, 7680-7687.

429 M. N. Jackson, S. Oh, C. J. Kaminsky, S. B. Chu, G. Zhang, J. T. Miller and Y. Surendranath, J. Am. Chem. Soc., 2018, 140, 1004-1010.

430 R. Wu, B. Xiao, Q. Gao, Y.-R. Zheng, X.-S. Zheng, J.-F. Zhu, M.-R. Gao and S.-H. Yu, Angew. Chem., Int. Ed., 2018, 57, 15445-15449.

431 X. Zhao, P. Pachfule, S. Li, J. R. J. Simke, J. Schmidt and A. Thomas, Angew. Chem., Int. Ed., 2018, 57, 8921-8926.

432 Y. Xiong, Y. Yang, F. J. DiSalvo and H. D. Abruña, J. Am. Chem. Soc., 2018, 140, 7248-7255.

433 Y. Duan, Z.-Y. Yu, S.-J. Hu, X.-S. Zheng, C.-T. Zhang, H.-H. Ding, B.-C. Hu, Q.-Q. Fu, Z.-L. Yu, X. Zheng, J.-F. Zhu, M.-R. Gao and S.-H. Yu, Angew. Chem., Int. Ed., 2019, 58, 15772-15777.

434 S. Li, C. Cheng, A. Sagaltchik, P. Pachfule, C. Zhao and A. Thomas, Adv. Funct. Mater., 2019, 29, 1807419.

435 X. Zhao, P. Pachfule, S. Li, T. Langenhahn, M. Ye, C. Schlesiger, S. Praetz, J. Schmidt and A. Thomas, J. Am. Chem. Soc., 2019, 141, 6623-6630.

436 H.-H. Li and S.-H. Yu, Adv. Mater., 2019, 31, 1803503.

437 Y. Lin, H. Wan, D. Wu, G. Chen, N. Zhang, X. Liu, J. Li, Y. Cao, G. Qiu and R. Ma, J. Am. Chem. Soc., 2020, 142, 7317-7321.

438 P. Xiong, B. Sun, N. Sakai, R. Ma, T. Sasaki, S. Wang, J. Zhang and G. Wang, Adv. Mater., 2020, 32, 1902654.

439 M.-Y. Ye, S. Li, X. Zhao, N. V. Tarakina, C. Teutloff, W. Y. Chow, R. Bittl and A. Thomas, Adv. Mater., 2020, 32, 1903942.

440 N. Dubouis and A. Grimaud, Chem. Sci., 2019, 10, 9165-9181.

441 N. M. Marković, H. A. Gasteiger, B. N. Grgur and P. N. Ross, J. Electroanal. Chem., 1999, 467, 157-163.
442 D. Strmcnik, D. F. van der Vliet, K. C. Chang, V. Komanicky, K. Kodama, H. You, V. R. Stamenkovic and N. M. Marković, J. Phys. Chem. Lett., 2011, 2, 2733-2736.

443 R. Subbaraman, D. Tripkovic, D. Strmenik, K.-C. Chang, M. Uchimura, A. P. Paulikas, V. Stamenkovic and N. M. Markovic, Science, 2011, 334, 1256-1260.

444 P. P. Lopes, D. Strmcnik, J. S. Jirkovsky, J. G. Connell, V. Stamenkovic and N. Markovic, Catal. Today, 2016, 262, 41-47.

445 J. Knossalla, P. Paciok, D. Göhl, D. Jalalpoor, E. Pizzutilo, A. M. Mingers, M. Heggen, R. E. Dunin-Borkowski, K. J. J. Mayrhofer, F. Schüth and M. Ledendecker, J. Am. Chem. Soc., 2018, 140, 15684-15689.

446 D. Göhl, A. Garg, P. Paciok, K. J. J. Mayrhofer, M. Heggen, Y. Shao-Horn, R. E. Dunin-Borkowski, Y. Román-Leshkov and M. Ledendecker, Nat. Mater., 2020, 19, 287-291.

447 M. Uchida, Y. Aoyama, N. Eda and A. Ohta, J. Electrochem. Soc., 1995, 142, 4143.

448 M. Uchida, Y. Fukuoka, Y. Sugawara, H. Ohara and A. Ohta, J. Electrochem. Soc., 1998, 145, 3708.

449 M. Lee, M. Uchida, D. A. Tryk, H. Uchida and M. Watanabe, Electrochim. Acta, 2011, 56, 4783-4790.

450 K. Shinozaki, H. Yamada and Y. Morimoto, J. Electrochem. Soc., 2011, 158, B467.

451 M. Uchida, Y.-C. Park, K. Kakinuma, H. Yano, D. A. Tryk, T. Kamino, H. Uchida and M. Watanabe, Phys. Chem. Chem. Phys., 2013, 15, 11236-11247.

452 Y.-C. Park, K. Kakinuma, M. Uchida, H. Uchida and M. Watanabe, Electrochim. Acta, 2014, 123, 84-92.

453 K. Kodama, R. Jinnouchi, A. Shinohara and Y. Morimoto, R\&D Rev. Toyota CRDL, 2018, 49, 1-11.

454 K. Momma and F. Izumi, J. Appl. Crystallogr., 2011, 44, 1272-1276.

455 R. Adzic, in Electrocatalysis, ed. J. Lipkowski and P. N. Ross, Wiley-VCH, USA, 1998, vol. 197, pp. 197-242.

456 B. E. Conway, in Interfacial Electrochemistry: Theory, Experiment, and Applications, ed. A. Wieckowski, Marcel Dekker, USA, 1999, ch. 9, pp. 131-150.

457 N. M. Marković, R. R. Adžić, B. D. Cahan and E. B. Yeager, J. Electroanal. Chem., 1994, 377, 249-259.

458 N. M. Markovic, H. A. Gasteiger and P. N. Ross, J. Phys. Chem., 1995, 99, 3411-3415.

459 N. M. Marković, H. A. Gasteiger and P. N. Ross, J. Phys. Chem., 1996, 100, 6715-6721.

460 N. M. Markovića, S. T. Sarraf, H. A. Gasteiger and P. N. Ross, J. Chem. Soc., Faraday Trans., 1996, 92, 3719-3725.

461 N. M. Marković, B. N. Grgur and P. N. Ross, J. Phys. Chem. $B$, 1997, 101, 5405-5413.

462 T. J. Schmidt, H. A. Gasteiger, G. D. Stäb, P. M. Urban, D. M. Kolb and R. J. Behm, J. Electrochem. Soc., 1998, 145, 2354.

463 N. Danilovic, R. Subbaraman, D. Strmcnik, K.-C. Chang, A. P. Paulikas, V. R. Stamenkovic and N. M. Markovic, Angew. Chem., Int. Ed., 2012, 51, 12495-12498.

464 T. J. Schmidt, U. A. Paulus, H. A. Gasteiger and R. J. Behm, J. Electroanal. Chem., 2001, 508, 41-47. 
465 U. A. Paulus, T. J. Schmidt, H. A. Gasteiger and R. J. Behm, J. Electroanal. Chem., 2001, 495, 134-145.

466 K. Ojha, N. Arulmozhi, D. Aranzales and M. T. M. Koper, Angew. Chem., Int. Ed., 2020, 59, 711-715.

467 Y. Fang, S. Y. Ding, M. Zhang, S. N. Steinmann, R. Hu, B. W. Mao, J. M. Feliu and Z. Q. Tian, J. Am. Chem. Soc., 2020, 142, 9439-9446.

468 B. M. Ocko, J. Wang, A. Davenport and H. Isaacs, Phys. Rev. Lett., 1990, 65, 1466-1469.

469 J. Wang, B. M. Ocko, A. J. Davenport and H. S. Isaacs, Phys. Rev. B: Condens. Matter Mater. Phys., 1992, 46, 10321-10338.

470 J. Lipkowski, L. Stolberg, D. F. Yang, B. Pettinger, S. Mirwald, F. Henglein and D. M. Kolb, Electrochim. Acta, 1994, 39, 1045-1056.

471 K.-i. Ataka, T. Yotsuyanagi and M. Osawa, J. Phys. Chem., 1996, 100, 10664-10672.

472 K.-i. Ataka and M. Osawa, Langmuir, 1998, 14, 951-959.

473 O. M. Magnussen, B. M. Ocko, M. J. Regan, K. Penanen, P. S. Pershan and M. Deutsch, Phys. Rev. Lett., 1995, 74, 4444-4447.

474 M. J. Regan, E. H. Kawamoto, S. Lee, P. S. Pershan, N. Maskil, M. Deutsch, O. M. Magnussen, B. M. Ocko and L. E. Berman, Phys. Rev. Lett., 1995, 75, 2498-2501.

475 M. R. Vogt, A. Lachenwitzer, O. M. Magnussen and R. J. Behm, Surf. Sci., 1998, 399, 49-69.

476 I. Burgess, C. A. Jeffrey, X. Cai, G. Szymanski, Z. Galus and J. Lipkowski, Langmuir, 1999, 15, 2607-2616.

477 A. Chen and J. Lipkowski, J. Phys. Chem. B, 1999, 103, 682-691.

478 O. M. Magnussen, J. Scherer, B. M. Ocko and R. J. Behm, J. Phys. Chem. B, 2000, 104, 1222-1226.

479 O. M. Magnussen, L. Zitzler, B. Gleich, M. R. Vogt and R. J. Behm, Electrochim. Acta, 2001, 46, 3725-3733.

480 O. M. Magnussen, Chem. Rev., 2002, 102, 679-726.

481 M. Nakamura, O. Endo, T. Ohta, M. Ito and Y. Yoda, Surf. Sci., 2002, 514, 227-233.

482 Z.-Q. Tian and B. Ren, Annu. Rev. Phys. Chem., 2004, 55, 197-229.

483 T. Tansel and O. M. Magnussen, Phys. Rev. Lett., 2006, 96, 026101.

484 D.-Y. Wu, J.-F. Li, B. Ren and Z.-Q. Tian, Chem. Soc. Rev., 2008, 37, 1025-1041.

485 B. J. Trześniewski, O. Diaz-Morales, D. A. Vermaas, A. Longo, W. Bras, M. T. M. Koper and W. A. Smith, J. Am. Chem. Soc., 2015, 137, 15112-15121.

486 M. C. Figueiredo, I. Ledezma-Yanez and M. T. M. Koper, ACS Catal., 2016, 6, 2382-2392.

487 J. H. K. Pfisterer, Y. Liang, O. Schneider and A. S. Bandarenka, Nature, 2017, 549, 74-77.

488 K. Wonner, M. V. Evers and K. Tschulik, J. Am. Chem. Soc., 2018, 140, 12658-12661.

489 Z. Jusys and R. J. Behm, ChemPhysChem, 2019, 20, 3276-3288. 490 Y. Yang, Y. Wang, Y. Xiong, X. Huang, L. Shen, R. Huang, H. Wang, J. P. Pastore, S.-H. Yu, L. Xiao, J. D. Brock, L. Zhuang and H. D. Abruña, J. Am. Chem. Soc., 2019, 141, 1463-1466.
491 M. V. Evers, M. Bernal, B. Roldan Cuenya and K. Tschulik, Angew. Chem., Int. Ed., 2019, 58, 8221-8225.

492 J.-C. Dong, M. Su, V. Briega-Martos, L. Li, J.-B. Le, P. Radjenovic, X.-S. Zhou, J. M. Feliu, Z.-Q. Tian and J.-F. Li, J. Am. Chem. Soc., 2020, 142, 715-719.

493 Z. Su, M. Karaskiewicz, J. Rogalski, R. Bilewicz and J. Lipkowski, J. Electroanal. Chem., 2020, 113820, DOI: 10.1016/j.jelechem.2020.113820.

494 J. Klein, V. Chesnyak, M. Löw, M. Schilling, A. K. Engstfeld and R. J. Behm, J. Am. Chem. Soc., 2020, 142, 1278-1286.

495 F. Buchner, M. Eckardt, T. Bohler, J. Kim, J. Gerlach, J. Schnaidt and R. J. Behm, ChemSusChem, 2020, 13, 3199-3211.

496 A. B. Anderson and T. V. Albu, Electrochem. Commun., 1999, 1, 203-206.

497 A. B. Anderson and T. V. Albu, J. Am. Chem. Soc., 1999, 121, 11855-11863.

498 A. B. Anderson, N. M. Neshev, R. A. Sidik and P. Shiller, Electrochim. Acta, 2002, 47, 2999-3008.

499 A. B. Anderson, Curr. Opin. Electrochem., 2017, 1, 27-33.

500 M. Andersen, S. V. Levchenko, M. Scheffler and K. Reuter, ACS Catal., 2019, 9, 2752-2759.

501 F. Tian, R. Jinnouchi and A. B. Anderson, J. Phys. Chem. C, 2009, 113, 17484-17492.

502 F. Abild-Pedersen, J. Greeley, F. Studt, J. Rossmeisl, T. R. Munter, P. G. Moses, E. Skúlason, T. Bligaard and J. K. Nørskov, Phys. Rev. Lett., 2007, 99, 016105.

503 J. Greeley and M. Mavrikakis, Nat. Mater., 2004, 3, 810-815.

504 A. B. Anderson, J. Uddin and R. Jinnouchi, J. Phys. Chem. C, 2010, 114, 14946-14952.

505 A. B. Anderson, R. Jinnouchi and J. Uddin, J. Phys. Chem. C, 2013, 117, 41-48.

506 R. Jinnouchi, K. Kodama, T. Hatanaka and Y. Morimoto, Phys. Chem. Chem. Phys., 2011, 13, 21070-21083.

507 A. B. Anderson, Phys. Chem. Chem. Phys., 2012, 14, 1330-1338.

508 F. Calle-Vallejo, J. Tymoczko, V. Colic, Q. H. Vu, M. D. Pohl, K. Morgenstern, D. Loffreda, P. Sautet, W. Schuhmann and A. S. Bandarenka, Science, 2015, 350, 185-189.

509 Y. Takahashi, A. Kumatani, H. Shiku and T. Matsue, Anal. Chem., 2017, 89, 342-357.

510 Y. Takagi, T. Uruga, M. Tada, Y. Iwasawa and T. Yokoyama, Acc. Chem. Res., 2018, 51, 719-727.

511 L. Yu, Y. Takagi, T. Nakamura, T. Sakata, T. Uruga, M. Tada, Y. Iwasawa, S. Masaoka and T. Yokoyama, J. Phys. Chem. C, 2019, 123, 603-611.

512 Y. Takahashi, Y. Kobayashi, Z. Wang, Y. Ito, M. Ota, H. Ida, A. Kumatani, K. Miyazawa, T. Fujita, H. Shiku, Y. E. Korchev, Y. Miyata, T. Fukuma, M. Chen and T. Matsue, Angew. Chem., Int. Ed., 2020, 59, 3601-3608.

513 H. Kiuchi, H. Niwa, M. Kobayashi, Y. Harada, M. Oshima, M. Chokai, Y. Nabae, S. Kuroki, M.-A. Kakimoto, T. Ikeda, K. Terakura and S. Miyata, Electrochim. Acta, 2012, 82, 291-295.

514 M. Pumera and H. Iwai, J. Phys. Chem. C, 2009, 113, 4401-4405. 
515 A. Ambrosi, S. Y. Chee, B. Khezri, R. D. Webster, Z. Sofer and M. Pumera, Angew. Chem., Int. Ed., 2012, 51, 500-503.

516 L. Wang, A. Ambrosi and M. Pumera, Angew. Chem., Int. Ed., 2013, 52, 13818-13821.

517 L. Wang, Z. Sofer and M. Pumera, ACS Nano, 2020, 14, 21-25.

518 E. Yeager, P. Krouse and K. V. Rao, Electrochim. Acta, 1964, 9, 1057-1070.

519 E. Yeager, Electrochim. Acta, 1984, 29, 1527-1537.

520 E. Yeager, J. Mol. Catal., 1986, 38, 5-25.

521 D. Guo, R. Shibuya, C. Akiba, S. Saji, T. Kondo and J. Nakamura, Science, 2016, 351, 361-365.

522 Y. Harada, J. Miyawaki, H. Niwa, K. Yamazoe, L. G. M. Pettersson and A. Nilsson, J. Phys. Chem. Lett., 2017, 8, 5487-5491.

523 K. H. Kim, H. Pathak, A. Späh, F. Perakis, D. Mariedahl, J. A. Sellberg, T. Katayama, Y. Harada, H. Ogasawara, L. G. M. Pettersson and A. Nilsson, Phys. Rev. Lett., 2017, 119, 075502.

524 A. Nilsson, J. LaRue, H. Öberg, H. Ogasawara, M. Dell'Angela, M. Beye, H. Öström, J. Gladh, J. K. Nørskov, W. Wurth, F. Abild-Pedersen and L. G. M. Pettersson, Chem. Phys. Lett., 2017, 675, 145-173.

525 C. W. Nicholson, A. Lücke, W. G. Schmidt, M. Puppin, L. Rettig, R. Ernstorfer and M. Wolf, Science, 2018, 362, 821-825.

526 H. Ali-Löytty, M. Hannula, M. Valden, A. Eilert, H. Ogasawara and A. Nilsson, J. Phys. Chem. C, 2019, 123, 25128-25134.

527 D. Friebel, H. Ogasawara and A. Nilsson, in Surface and Interface Science, ed. K. Wandelt, John Wiley and Sons, Weinheim, 2020, ch. 58, pp. 733-772, DOI: 10.1002/ 9783527680603.ch58.

528 M. Ahmed, K. I. Inoue, S. Nihonyanagi and T. Tahara, Angew. Chem., Int. Ed., 2020, 59, 9498-9505.

529 S. Kawai, T. Glatzel, S. Koch, B. Such, A. Baratoff and E. Meyer, Phys. Rev. Lett., 2009, 103, 220801.

530 T. Kumagai, F. Hanke, S. Gawinkowski, J. Sharp, K. Kotsis, J. Waluk, M. Persson and L. Grill, Nat. Chem., 2014, 6, 41-46.

531 S. Kawai, S. Saito, S. Osumi, S. Yamaguchi, A. S. Foster, P. Spijker and E. Meyer, Nat. Commun., 2015, 6, 8098.

532 H. Imada, K. Miwa, M. Imai-Imada, S. Kawahara, K. Kimura and Y. Kim, Nature, 2016, 538, 364-367.

533 E. Kazuma, J. Jung, H. Ueba, M. Trenary and Y. Kim, Science, 2018, 360, 521-526.

534 R. Evans, Adv. Phys., 1979, 28, 143-200.

535 R. Evans, in Fundamentals of Inhomogeneous Fluids, ed. D. Henderson, Dekker, New York, 1992, ch. 3, pp. 85-175.

536 H. Park, N. Kumar, M. Melander, T. Vegge, J. M. Garcia Lastra and D. J. Siegel, Chem. Mater., 2018, 30, 915-928.

537 T. Engel and G. Ertl, in Advances in Catalysis, ed. D. D. Eley, H. Pines and P. B. Weez, Academic Press, 1979, vol. 28, pp. 1-78.

538 M. Eiswirth and G. Ertl, Surf. Sci., 1986, 177, 90-100.

539 G. Ertl, Adv. Catal., 1990, 37, 213-277.
540 K. Krischer, M. Eiswirth and G. Ertl, J. Chem. Phys., 1992, 96, 9161-9172.

541 R. Imbihl and G. Ertl, Chem. Rev., 1995, 95, 697-733.

542 M. Kim, M. Bertram, M. Pollmann, A. V. Oertzen, A. S. Mikhailov, H. H. Rotermund and G. Ertl, Science, 2001, 292, 1357-1360.

543 J. Clavilier, R. Faure, G. Guinet and R. Durand, J. Electroanal. Chem. Interfacial Electrochem., 1980, 107, 205-209.

544 J. Clavilier, J. Electroanal. Chem. Interfacial Electrochem., 1980, 107, 211-216.

545 J. Clavilier, D. Armand, S. G. Sun and M. Petit, J. Electroanal. Chem. Interfacial Electrochem., 1986, 205, 267-277.

546 J. Clavilier, R. Albalat, R. Gomez, J. M. Orts, J. M. Feliu and A. Aldaz, J. Electroanal. Chem., 1992, 330, 489-497.

547 J. Hotlos, O. M. Magnussen and R. J. Behm, Surf. Sci., 1995, 335, 129-144.

548 F. A. Möller, O. M. Magnussen and R. J. Behm, Phys. Rev. Lett., 1996, 77, 5249-5252.

549 F. A. Möller, O. M. Magnussen and R. J. Behm, Phys. Rev. Lett., 1996, 77, 3165-3168.

550 F. A. Möller, J. Kintrup, A. Lachenwitzer, O. M. Magnussen and R. J. Behm, Phys. Rev. B: Condens. Matter Mater. Phys., 1997, 56, 12506-12518.

551 M. T. M. Koper and R. A. van Santen, J. Electroanal. Chem., 1999, 476, 64-70.

552 N. P. Lebedeva, M. T. M. Koper, E. Herrero, J. M. Feliu and R. A. van Santen, J. Electroanal. Chem., 2000, 487, 37-44.

553 E. Herrero, L. J. Buller and H. D. Abruña, Chem. Rev., 2001, 101, 1897-1930.

554 N. P. Lebedeva, M. T. M. Koper, J. M. Feliu and R. A. van Santen, J. Phys. Chem. B, 2002, 106, 12938-12947.

555 M. D. Maciá, J. M. Campiña, E. Herrero and J. M. Feliu, J. Electroanal. Chem., 2004, 564, 141-150.

556 T. E. Shubina, C. Hartnig and M. T. M. Koper, Phys. Chem. Chem. Phys., 2004, 6, 4215-4221.

557 A. Kuzume, E. Herrero and J. M. Feliu, J. Electroanal. Chem., 2007, 599, 333-343.

558 J. Solla-Gullón, P. Rodríguez, E. Herrero, A. Aldaz and J. M. Feliu, Phys. Chem. Chem. Phys., 2008, 10, 1359-1373.

559 S. C. S. Lai and M. T. M. Koper, Phys. Chem. Chem. Phys., 2009, 11, 10446-10456.

560 K. J. P. Schouten, Z. Qin, E. Pérez Gallent and M. T. M. Koper, J. Am. Chem. Soc., 2012, 134, 9864-9867.

561 E. Herrero and J. M. Feliu, Curr. Opin. Electrochem., 2018, 9, 145-150.

562 L. Jacobse, Y.-F. Huang, M. T. M. Koper and M. J. Rost, Nat. Mater., 2018, 17, 277-282.

563 V. Briega-Martos, E. Herrero and J. M. Feliu, Curr. Opin. Electrochem., 2019, 17, 97-105.

564 R. Martínez-Hincapié, V. Climent and J. M. Feliu, Curr. Opin. Electrochem., 2019, 14, 16-22.

565 V. Climent and J. Feliu, Annu. Rev. Anal. Chem., 2020, 13, 201-222.

566 F. Calle-Vallejo, M. T. M. Koper and A. S. Bandarenka, Chem. Soc. Rev., 2013, 42, 5210-5230. 
567 A. S. Bandarenka, A. S. Varela, M. Karamad, F. CalleVallejo, L. Bech, F. J. Perez-Alonso, J. Rossmeisl, I. E. L. Stephens and I. Chorkendorff, Angew. Chem., Int. Ed., 2012, 51, 11845-11848.

568 V. Climent and J. M. Feliu, J. Solid State Electrochem., 2011, 15, 1297.

569 Z. W. Seh, J. Kibsgaard, C. F. Dickens, I. Chorkendorff, J. K. Nørskov and T. F. Jaramillo, Science, 2017, 355, eaad4998.

570 S. Hammes-Schiffer, Faraday Discuss., 2020, 221, 582-588.

571 S. C. Althorpe, W. Barford, J. Blumberger, C. Bungey, I. Burghardt, A. Datta, S. Ghosh, S. Giannini, T. Grünbaum, S. Habershon, S. Hammes-Schiffer, S. Hay, S. Iyengar, G. Jones, A. Kelly, K. Komarova, J. Lawrence, Y. Litman, J. Mannouch, D. Manolopoulos, C. Martens, R. J. Maurer, M. Melander, M. Rossi, K. Sakaushi, M. Saller, A. Schile, S. Sturniolo, G. Trenins and G. Worth, Faraday Discuss., 2020, 221, 564-581.

572 A. M. Alvertis, W. Barford, S. Bourne Worster, I. Burghardt, A. Chin, A. Datta, A. Dijkstra, T. Fay, H. Fielding, T. Grünbaum, S. Habershon, S. Hammes-Schiffer, S. Iyengar, A. R. Jones, K. Komarova, J. Léonard, Y. Litman, D. Picconi, D. Plant, A. Schile, G. D. Scholes, J. Segarra-Martí, F. Segatta, A. Troisi and G. Worth, Faraday Discuss., 2020, 221, 322-349.

573 A. M. Alvertis, W. Barford, S. Bourne Worster, I. Burghardt, A. Datta, A. Dijkstra, T. Fay, S. Ghosh, T. Grünbaum, S. Habershon, P. J. Hore, D. Hutchinson, S. Iyengar, A. R. Jones, G. Jones, K. Komarova, J. Lawrence, J. Léonard, Y. Litman, J. Mannouch, D. Manolopoulos, C. Martens, M. Mondelo-Martell, D. Picconi, D. Plant, K. Sakaushi, M. A. C. Saller, A. Schile, G. D. Scholes, J. Segarra-Martí, F. Segatta, A. Troisi and G. Worth, Faraday Discuss., 2020, 221, 168-201.

574 S. C. Althorpe, A. M. Alvertis, W. Barford, R. L. Benson, I. Burghardt, S. Giannini, S. Habershon, S. HammesSchiffer, S. Hay, S. Iyengar, A. Kelly, K. Komarova, J. Lawrence, Y. Litman, C. Martens, R. J. Maurer, D. Plant, M. Rossi, K. Sakaushi, A. Schile, S. Sturniolo, D. P. Tew,
G. Trenins and G. Worth, Faraday Discuss., 2020, 221, 478-500.

575 V. R. Stamenkovic, B. S. Mun, K. J. J. Mayrhofer, P. N. Ross and N. M. Markovic, J. Am. Chem. Soc., 2006, 128, 8813-8819.

576 V. R. Stamenkovic, B. Fowler, B. S. Mun, G. Wang, P. N. Ross, C. A. Lucas and N. M. Marković, Science, 2007, 315, 493-497.

577 V. R. Stamenkovic, B. S. Mun, M. Arenz, K. J. J. Mayrhofer, C. A. Lucas, G. Wang, P. N. Ross and N. M. Markovic, Nat. Mater., 2007, 6, 241-247.

578 C. Chen, Y. Kang, Z. Huo, Z. Zhu, W. Huang, H. L. Xin, J. D. Snyder, D. Li, J. A. Herron, M. Mavrikakis, M. Chi, K. L. More, Y. Li, N. M. Markovic, G. A. Somorjai, P. Yang and V. R. Stamenkovic, Science, 2014, 343, 1339-1343.

579 Z. He, S. D. Minteer and L. T. Angenent, Environ. Sci. Technol., 2005, 39, 5262-5267.

580 D. R. Lovley and K. P. Nevin, Curr. Opin. Biotechnol., 2011, 22, 441-448.

581 K. Dolch, J. Danzer, T. Kabbeck, B. Bierer, J. Erben, A. H. Förster, J. Maisch, P. Nick, S. Kerzenmacher and J. Gescher, Bioresour. Technol., 2014, 157, 284-292.

582 K. K. Sakimoto, A. B. Wong and P. Yang, Science, 2016, 351, 74-77.

583 K. K. Sakimoto, N. Kornienko and P. Yang, Acc. Chem. Res., 2017, 50, 476-481.

584 Z. Ji, H. Zhang, H. Liu, O. M. Yaghi and P. Yang, Proc. Natl. Acad. Sci. U. S. A., 2018, 115, 10582-10587.

585 K. K. Sakimoto, N. Kornienko, S. Cestellos-Blanco, J. Lim, C. Liu and P. Yang, J. Am. Chem. Soc., 2018, 140, 1978-1985.

586 N. Heidary, N. Kornienko, S. Kalathil, X. Fang, K. H. Ly, H. F. Greer and E. Reisner, J. Am. Chem. Soc., 2020, 142, 5194-5203.

587 J. Z. Zhang and E. Reisner, Nat. Rev. Chem., 2020, 4, 6-21.

588 E. M. Gaffney, M. Grattieri, Z. Rhodes and S. D. Minteer, J. Electrochem. Soc., 2020, 167, 065502.

589 D. M. Kolb and J. Schneider, Electrochim. Acta, 1986, 31, 929-936.

590 D. M. Kolb, Prog. Surf. Sci., 1996, 51, 109-173. 